\title{
Evaluating EYM amplitudes in four dimensions by refined graphic expansion
}

\author{
Hongxiang Tian, ${ }^{a, 1}$ Enze Gong, ${ }^{a, b}$ Chongsi $\mathbf{X i e}^{a}$ and $\mathrm{Yi}$-Jian Du ${ }^{a, c}$ \\ ${ }^{a}$ School of Physics and Technology, Wuhan University, \\ No. 299 Bayi Road, Wuhan 430072, China \\ ${ }^{b}$ Department of Physics and Astronomy, Uppsala University, \\ 75108 Uppsala, Sweden \\ ${ }^{c}$ Suzhou Institute of Wuhan University, \\ No. 377 Linquan Street, Suzhou 215123, China \\ E-mail: thx_world@whu.edu.cn, Enze.Gong.2675@student.uu.se, \\ chongsi.xie@whu.edu.cn, yijian.du@whu.edu.cn
}

ABSTRACT: The recursive expansion of tree level multitrace Einstein-Yang-Mills (EYM) amplitudes induces a refined graphic expansion, by which any tree-level EYM amplitude can be expressed as a summation over all possible refined graphs. Each graph contributes a unique coefficient as well as a proper combination of color-ordered Yang-Mills (YM) amplitudes. This expansion allows one to evaluate EYM amplitudes through YM amplitudes, the latter have much simpler structures in four dimensions than the former. In this paper, we classify the refined graphs for the expansion of EYM amplitudes into $\mathrm{N}^{k} \mathrm{MHV}$ sectors. Amplitudes in four dimensions, which involve $k+2$ negative-helicity particles, at most get non-vanishing contribution from graphs in $\mathrm{N}^{k^{\prime}\left(k^{\prime} \leq k\right)} \mathrm{MHV}$ sectors. By the help of this classification, we evaluate the non-vanishing amplitudes with two negative-helicity particles in four dimensions. We establish a correspondence between the refined graphs for single-trace amplitudes with $\left(g_{i}^{-}, g_{j}^{-}\right)$or $\left(h_{i}^{-}, g_{j}^{-}\right)$configuration and the spanning forests of the known Hodges determinant form. Inspired by this correspondence, we further propose a symmetric formula of double-trace amplitudes with $\left(g_{i}^{-}, g_{j}^{-}\right)$configuration. By analyzing the cancellation between refined graphs in four dimensions, we prove that any other tree amplitude with two negative-helicity particles has to vanish.

KEYwords: Scattering Amplitudes, Gauge Symmetry

ARXIV EPRINT: 2101.02962

\footnotetext{
${ }^{1}$ The unusual ordering of authors is to let authors get proper recognition of contributions under the practice in China.
} 


\section{Contents}

1 Introduction 1

2 Sectors of tree-level EYM amplitudes 4

2.1 The expansion of single-trace MHV amplitudes $\quad 7$

$\begin{array}{lll}2.2 & \text { The expansion of double-trace MHV amplitudes } & 7\end{array}$

3 Single-trace MHV amplitudes with $\left(g_{i}^{-}, g_{j}^{-}\right)$and $\left(h_{i}^{-}, g_{j}^{-}\right) \quad 8$

3.1 Single-trace amplitudes with $\left(g_{i}^{-}, g_{j}^{-}\right)$configuration $\quad 9$

$\begin{array}{ll}3.2 & \text { Single-trace amplitudes with }\left(h_{i}^{-}, g_{j}^{-}\right) \text {configuration } \\ 3.3 & \text { Comments }\end{array}$

3.3 Comments 12

4 Double-trace MHV amplitudes with $\left(g_{i}^{-}, g_{j}^{-}\right)$configuration 13

$\begin{array}{lll}4.1 \text { Symmetries of the formula } & 14\end{array}$

$\begin{array}{ll}4.2 \text { An example study } & 17\end{array}$

$\begin{array}{lll}4.3 & \text { The general proof } & 21\end{array}$

5 The vanishing configurations $\quad 25$

5.1 Helpful properties of refined graphs for amplitudes with two negative-helicity particles

5.2 The vanishing amplitudes 32

6 Conclusions and further discussions $\quad 34$

A Sectors in the graphic expansion of EYM amplitudes $\quad 35$

A.1 The construction of a graph $\mathcal{F} \in \mathcal{F}[k, m]$ in the $\mathrm{N}^{k} \mathrm{MHV}$ sector 35

A.2 Coefficient, sign and permutations associated to $\mathcal{F} \quad 38$

A.3 Graphs in the MHV sectors of single- and double-trace amplitudes 38

$\begin{array}{ll}\text { B Spinor helicity formalism and helpful identities } & 38\end{array}$

$\begin{array}{lll}\text { B.1 Spinor helicity formalism } & 38\end{array}$

$\begin{array}{lll}\text { B.2 Useful identities for Parke-Taylor factors } & 39\end{array}$

B.3 Parke-Taylor formula and Hodges formula 40

\section{Introduction}

The expansion of Einstein-Yang-Mills $(\mathrm{EYM})^{1}$ amplitudes in terms of pure Yang-Mills (YM) amplitudes was firstly suggested in [1], where a tree-level single-trace EYM amplitude with one graviton and $n$ gluons was written as a combination of $n+1$ gluon

\footnotetext{
${ }^{1}$ In this paper, EYM theory always means the theory which also involves antisymmetric B field and dilation, while the external particles of amplitudes can only be gravitons and gluons.
} 
color-ordered YM amplitudes. This observation was then extended to amplitudes with a few gravitons and/or gluon traces in many literatures, see e.g., [2-4]. A general study of the expansion of single-trace amplitudes can be found in [5-7]. As pointed in [5], a single-trace EYM amplitude could be expanded recursively in terms of EYM amplitudes with fewer gravitons. Thus, the pure-YM expansion can be obtained by applying the recursive expansion iteratively. Along this line, the general formulas for recursive expansion of an arbitrary tree-level multitrace EYM amplitude were established in [8]. Further applications and generalizations of this recursive expansion have been investigated, including the symmetry-induced identities [5, 8-10], the proof [11] of the equivalence between distinct approaches to amplitudes in nonlinear sigma model [12-14], the construction of polynomial Bern-Carrasco-Johansson (BCJ) [15] numerators [5, 14], the expansion into BCJ basis $[16,17]$ as well as the generalization to amplitudes in other theories $[18,19]$.

Among these progresses, a refined graphic rule which conveniently expands an EYM amplitude by summing over a set of so-called refined graphs has been invented [9, 10]. Each tree graph in this expansion provides a coefficient (expressed by a product of the Lorentz contractions of external polarizations and/or momenta) as well as a proper combination of color-ordered YM amplitudes. This refined graphic expansion was already shown to be a powerful tool in the study of the relationship between symmetry-induced identities and BCJ relations [9, 10].

On the other hand, EYM amplitudes with particular helicity configurations in four dimensions was also studied in many work. By substituting solutions to scattering equations directly into the Cachazo-He-Yuan formula [20-24], the single-trace EYM amplitudes with the maximally-helicity-violating (MHV) configuration, which involves only two negativehelicity particles $\left(i^{-}, j^{-}\right)(i, j$ can be either (i) two gluons or (ii) one graviton and one gluon), have been shown to satisfy a compact formula [25] that is expressed by the well known Hodges determinant [26]. Through a spanning forest expansion [27], this compact formula was shown (see [25]) to be equivalent to a generating functional formula which was proposed in earlier work [28-30]. At double-trace level, a symmetric formula for the MHV amplitudes with only external gluons was founded [23]. In addition, explicit results of fiveand six-point examples of double-trace MHV amplitudes with one and two gravitons were also provided in [24]. However, it is still lack of a general symmetric formula of double-trace MHV amplitudes with an arbitrary number of gravitons.

Since YM amplitudes have much simpler forms than EYM ones, the refined graphic expansion may provide a new approach to the EYM amplitudes in four dimensions. In this paper, we evaluate the EYM amplitudes in four dimensions by the refined graphic rule. To achieve this, we classify refined graphs into $\mathrm{N}^{k} \mathrm{MHV}$ sectors and demonstrate that the $\mathrm{N}^{k} \mathrm{MHV}$ amplitudes with $(k+2)$ negative-helicity particles at most get nonvanishing contributions from graphs in the $\mathrm{N}^{k^{\prime}\left(k^{\prime} \leq k\right)} \mathrm{MHV}$ sectors. When $k=0$, the nonvanishing MHV amplitudes can only get contribution from the refined graphs in the MHV sector and the corresponding YM amplitudes in the expansion satisfy Parke-Taylor formula [31]. For single-trace MHV amplitudes with two negative-helicity gluons or those with one-negative helicity graviton and one negative-helicity gluon, a correspondence between the refined graphs and the spanning forests of the Hodges determinant is established. Hence this approach precisely reproduces the known results [25]. Inspired by this correspondence, 
we further propose a spanning forest formula of double-trace MHV amplitudes with two negative-helicity gluons, in which the following symmetries are manifest: (i). invariance under permutations of gravitons, (ii). invariance under exchanging the two traces, (iii). the cyclic symmetry of each trace. By an analysis on the cancellation between graphs, we show that all other tree amplitudes with two negative-helicity particles have to vanish.

The structure of this paper is organized as follows. In section 2, we provide the construction rule for the MHV sector of tree level single- and double-trace EYM amplitudes. By the help of the spinor-helicity formalism [32] in four dimensions and helpful identities between Parke-Taylor factors, we establish a correspondence between the refined graphs in the MHV sector for single-trace amplitudes and the spanning forests of Hodges determinant in section 3. In section 4, a symmetric formula of double-trace MHV amplitudes with two negative-helicity gluons is derived. Other tree amplitudes with two negative helicity particles are proven to vanish in section 5 . This work is summarized in section 6 . The refined graphic rule for the $\mathrm{N}^{k} \mathrm{MHV}$ sector of an $m$-trace amplitude is provided in appendix A. Spinor-helicity formalism and helpful identities are reviewed in appendix B.

Convention of notations. Convention of notations is displayed as follows. ${ }^{2}$

Permutations. We use boldface Greek letters $\beta, \gamma, \sigma$, or boldface uppercase Latin letters $\boldsymbol{X}$ and $\boldsymbol{Y}$ to denote permutations (or in other words ordered sets). The $i$-th element in a permutation $\beta$ is usually denoted by $\beta_{i}$, while the position of an element $a \in \beta$ is expressed by $\beta^{-1}(a)$. The condition $\beta^{-1}(a)<\beta^{-1}(b)$ is written as $a \prec b$ in some places for short. The inverse permutation of $\beta$ is denoted by $\beta^{T}$. Shuffle permutations of two ordered sets $\boldsymbol{X}$ and $\boldsymbol{Y}$ are written as $\boldsymbol{X} \amalg \boldsymbol{Y}$. Number of elements in $\boldsymbol{X}$ is denoted as $|\boldsymbol{X}|$.

Gravitons, gluon traces and amplitudes. We denote gluon traces by boldface numbers $\mathbf{1}, \mathbf{2}, \ldots$, or boldface lowercase Latin letters $\boldsymbol{t}, \boldsymbol{i} \ldots$ If a trace $\boldsymbol{i}$ can be written as $\boldsymbol{i}=$ $\left\{a_{i}, \boldsymbol{X}_{i}, b_{i}, \boldsymbol{Y}_{i}\right\}$, we define $\mathrm{KK}\left[\boldsymbol{i}, a_{i}, b_{i}\right] \equiv \boldsymbol{X}_{i} ш \boldsymbol{Y}_{i}^{T}$ and $(-1)^{\left|\boldsymbol{i}, a_{i}, b_{i}\right|} \equiv(-1)^{\left|\boldsymbol{Y}_{i}\right|}$. A multitrace EYM amplitude is generally denoted by $A(1,2, \ldots, r|\mathbf{2}| \ldots \mid \boldsymbol{m} \| \mathrm{H})$, where $\mathrm{H}$ stands for the graviton set with gravitons $h_{1}, h_{2}, \ldots$, while $1,2, \ldots, \boldsymbol{m}$ are the gluon traces. Gluons in the trace 1 are explicitly denoted by $1, \ldots, r$. When we study double trace amplitudes, we use $x_{1}, \ldots, x_{r}$ and $y_{1}, \ldots, y_{s}$ to denote the gluons in traces $\mathbf{1}$ and 2 respectively. The set of all gravitons and gluon traces is denoted by $\mathcal{H}$.

Refined graphs and spanning forests. In this paper, graphs constructed by refined graphic rule are mentioned as refined graphs and denoted by $\mathcal{F}$, while graphs that describe helicity amplitudes in the final formula are mentioned as spanning forests and denoted by $\mathcal{G}$. When we fix 1 and $r$ as the first and the last elements, the set of permutations (of other nodes) established by a refined graph $\mathcal{F}$ is denoted by $\left.\mathcal{F}\right|_{1} \backslash\{1, r\}$. Components of a graph $\mathcal{F}$ are denoted by $\mathscr{C}_{i}$, while a chain of components is denoted by $\mathbb{C H}$. We use $\mathrm{R}$ to stands for the reference order of gravitons and traces in the refined graphic rule and use $\mathrm{R}_{\mathscr{C}}$ to denote the reference order of components. The set of refined graphs which involve $m$ traces and belong to the $\mathrm{N}^{k} \mathrm{MHV}$ sector is denoted by $\mathcal{F} \in \mathcal{F}[k, m]$. Disjoint union of graphs are denoted by $\oplus$.

\footnotetext{
${ }^{2}$ Most notations follow from the paper [10].
} 


\begin{tabular}{|c|c|c|c|}
\hline$b$ & $b$ & $b$ & \\
\hline $\overrightarrow{\epsilon_{a} \cdot \epsilon_{b}}$ & $\overrightarrow{\epsilon_{a} \cdot \vec{k}_{b}}$ & $\overleftrightarrow{k_{a} \cdot \vec{k}_{b}}$ & \\
\hline $\begin{array}{l}\text { Type-1 } \\
\text { (a) }\end{array}$ & $\begin{array}{l}\text { Type-2 } \\
\text { (b) }\end{array}$ & $\begin{array}{c}\text { Type-3 } \\
\text { (c) }\end{array}$ & $\begin{array}{c}\text { Type-4 } \\
\text { (d) }\end{array}$ \\
\hline
\end{tabular}

Figure 1. Four types of lines in the refined graphic rule for multitrace EYM amplitudes.

\section{Sectors of tree-level EYM amplitudes}

As pointed out in $[8,10]$, an arbitrary tree level EYM amplitude $A(1,2, \ldots, r|\mathbf{2}| \ldots \mid \boldsymbol{m} \| \mathrm{H})$ with the graviton set $\mathbf{H}$ and the gluon traces $\mathbf{1}=\{1,2, \ldots, r\}, 2, \ldots, \boldsymbol{m}$ can be generally expressed as a combination of tree level color-ordered pure YM amplitudes

$$
A(1,2, \ldots, r|\mathbf{2}| \ldots \mid \boldsymbol{m} \| \mathrm{H})=\sum_{\mathcal{F}}(-)^{\mathcal{F}} \mathcal{C}^{\mathcal{F}}\left[\sum_{\boldsymbol{\sigma} \in \mathcal{F} \mid 1 \backslash\{1, r\}} A(1, \boldsymbol{\sigma}, r)\right],
$$

where we have summed over all possible connected tree graphs $\mathcal{F}$ which are constructed according to the refined graphic rule $[8,10]$. A tree graph $\mathcal{F}$ defines a coefficient $\mathcal{C}^{\mathcal{F}}$ as well as a combination of color-ordered YM amplitudes $A(1, \sigma, r)$. The polarization tensor $\epsilon_{h}^{\mu \nu}$ of a graviton splits into two polarization vectors $\epsilon_{h}^{\mu} \epsilon_{h}^{\nu}$. One of them is absorbed into the coefficient $\mathcal{C}^{\mathcal{F}}$, the other is considered as the polarization vector of a gluon which is involved in the pure YM amplitude $A(1, \boldsymbol{\sigma}, r)$. In each graph $\mathcal{F}$, all gluons and gravitons are expressed by nodes. As shown by figure 1, distinct types of lines between nodes are introduced: (a). A type-1 line represents an $\epsilon \cdot \epsilon$ factor between two gravitons; (b). A type-2 line denotes an $\epsilon \cdot k$ factor ( $k^{\mu}$ denotes the momentum of a node) between a graviton and a graviton/gluon; (c). A type-3 line stands for a $k \cdot k$ factor between any two nodes; (d). A type- 4 line is introduced to record the relative order of two adjacent gluons in a gluon trace but does not contribute any nontrivial kinematic factor. The expansion coefficient $\mathcal{C}^{\mathcal{F}}$ is then given by the product of factors defined by all lines in $\mathcal{F}$, while a proper sign $(-)^{\mathcal{F}}$ is also defined (see appendix A). Permutations $\left.\sigma \in \mathcal{F}\right|_{1} \backslash\{1, r\}$ established by the graph $\mathcal{F}$ are given by the following two steps: (i). If $a$ and $b$ are two adjacent nodes which satisfy the condition that $a$ is nearer to the gluon 1 than $b$, we have $\sigma^{-1}(a)<\sigma^{-1}(b)$ where $\sigma^{-1}(a)$ and $\sigma^{-1}(b)$ respectively denote the positions of $a$ and $b$ in $\sigma .{ }^{3}$ (ii). If two subtree structures are attached to a same node, we shuffle the corresponding permutations together such that the permutation in each subtree is preserved.

In order to analyze helicity amplitudes with $m$ traces in four dimensions, we denote the set of all graphs involving $k$ type- 1 lines (i.e. $\epsilon \cdot \epsilon$ lines) and $m$ gluon traces by $\mathcal{F}[k, m]$. We further define the $\mathrm{N}^{k} \mathrm{MHV}$ sector $I[k, m](k \geq 0)$ of the expansion (2.1) by the total contribution of graphs in $\mathcal{F}[k, m]$ as follows:

$$
I[k, m] \equiv \sum_{\mathcal{F} \in \mathcal{F}[k, m]}(-)^{\mathcal{F}} \mathcal{C}^{\mathcal{F}} \sum_{\left.\boldsymbol{\sigma} \in \mathcal{F}\right|_{1} \backslash\{1, r\}} A(1, \boldsymbol{\sigma}, r) .
$$

\footnotetext{
${ }^{3}$ Supposing that the position of $a$ in $\boldsymbol{\sigma}$ is $j$, we have $a=\sigma_{j} \equiv \sigma(j)$, hence it is reasonable to define $j=\sigma^{-1}(a)$.
} 
The expansion (2.1) is then given by summing over all possible $k$

$$
A(1,2, \ldots, r|\mathbf{2}| \ldots \mid \boldsymbol{m} \| \mathbf{H})=\sum_{k} I[k, m] .
$$

The general refined graphic rule for constructing the $\mathrm{N}^{k} \mathrm{MHV}$ sector is provided in appendix A. In the following, we illustrate that an $N^{k} M H V$ amplitude in four dimensions can at most get nonzero contribution from graphs in the $N^{k^{\prime}\left(k^{\prime} \leq k\right)} M H V$ sector.

In four dimensions, both the momentum of a graviton/gluon and the (half) polarization of a graviton can be expressed according to the spinor-helicity formalism [32] (see appendix B). The Lorentz contractions between momenta and/or polarizations are then expressed by spinor products, see eq. (B.3). In this paper, the reference momenta of all those gravitons with the same helicity are chosen as the same one. With this choice of gauge, the contractions between the (half) polarizations of any two gravitons with the same helicity must vanish. The only possible remaining contractions between 'half' polarizations must have the form $\epsilon_{h_{i}}^{+} \cdot \epsilon_{h_{j}}^{-}$. For an $m$-trace $\mathrm{N}^{k} \mathrm{MHV}$ amplitude with $l$ negative-helicity gravitons and $k-l+2$ negative-helicity gluons, the number of factors $\epsilon_{h_{i}}^{+} \cdot \epsilon_{h_{j}}^{-}$in the coefficient $\mathcal{C}^{\mathcal{F}}$ is at most $l$. This implies only those graphs $\mathcal{F} \in \mathcal{F}\left[k^{\prime}, m\right]$, which contain $k^{\prime}\left(k^{\prime} \leq l\right)$ type-1 lines, may provide nonvanishing contributions to an $\mathrm{N}^{k} \mathrm{MHV}$ amplitude in four dimensions. The number of type-1 lines can be further reduced when the following two facts are considered:

(i). It has been known that an EYM amplitude where all the negative-helicity particles are gravitons (in other words all gluons carry the same helicity) must vanish. Thus amplitudes in the case of $l=k+2$, for which a graph at most involves $l=k+2$ nontrivial type-1 lines, have to vanish. This fact can be easily verified by either the KawaiLewellen-Tye relation [30, 33] or Britto-Carasso-Feng-Witten recursion [34, 35].

(ii). When the reference momentum of all positive-helicity gravitons are chosen as the momentum of one of the negative-helicity gravitons, say $h_{i}$, the maximal number of type-1 lines in a graph is further reduced by one because $\epsilon_{h_{i}}^{-}(p) \cdot \epsilon_{h_{j}}^{+}\left(k_{h_{i}}\right)=0$. Thus amplitudes with $l=k+1$ can only get nonzero contributions from graphs $\mathcal{F} \in \mathcal{F}\left[k^{\prime}, m\right]\left(k^{\prime} \leq k\right)$.

Therefore, the nonvanishing EYM amplitudes with $k+2$ negative-helicity particles can at most get nonzero contributions from the graphs in the $\mathrm{N}^{k^{\prime}\left(k^{\prime} \leq k\right)} \mathrm{MHV}$ sector.

The fact that an $\mathrm{N}^{k} \mathrm{MHV}$ amplitude only gets contribution from terms involving $(\epsilon$. $\epsilon)^{k^{\prime}}\left(k^{\prime} \leq k\right)$ can be conveniently understood from another point of view, by the help of Britto-Cachazo-Feng-Witten (BCFW) recursion [34, 35]. According to BCFW recursion, an EYM amplitude $A$ with $k+2$ negative-helicity particles (gravitons and gluons) can be expressed as $A \sim \sum \widehat{A}_{L} \frac{1}{P^{2}} \widehat{A}_{R}$, where $\widehat{A}_{L}$ and $\widehat{A}_{R}$ are on-shell amplitudes with fewer particles. Supposing the particles with shifted momenta are two gluons in a same trace, as shown in the paper [8], the internal particle then can only be a gluon which does not contribute to the number of type- 1 lines (i.e. $\epsilon \cdot \epsilon$ factors). Assuming that $k_{L}\left(k_{R}\right)$ of the $k+2$ external negative-helicity particles in $A$ are distributed into $\widehat{A}_{L}\left(\widehat{A}_{R}\right)$, we have $k_{L}+k_{R}=k+2$. When we expand both sub-amplitudes according to eq. (2.1) and choose 


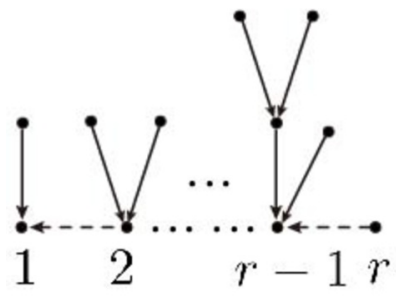

(a)

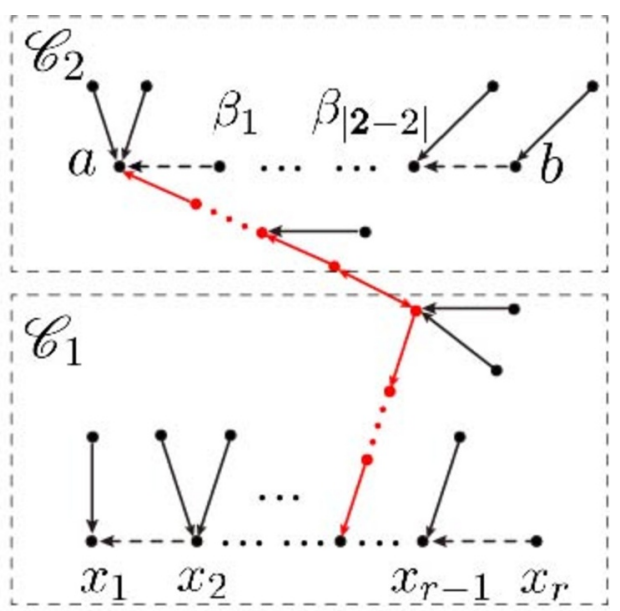

(b)

Figure 2. (a): a typical graph for the MHV sector of single-trace EYM amplitudes. (b): a typical graph for the MHV sector of double-trace EYM amplitudes.

the reference momenta of all gravitons with the same helicity as the same one (the reference momentum for positive-helicity particles is chosen as the momentum of a negative-helicity graviton in each sub-amplitude), the largest number of $(\epsilon \cdot \epsilon)$ factors in $\widehat{A}_{L}$ and $\widehat{A}_{R}$ are apparently $k_{L}-1$ and $k_{R}-1$. Consequently, the number of $(\epsilon \cdot \epsilon)$ factors in any term of the BCFW expression is at most $\left(k_{L}-1\right)+\left(k_{R}-1\right)=k$ which is the expected result. We emphasize that the choice of gauge in this BCFW approach (where the reference momenta in distinct sub-amplitudes are chosen differently) differs from that in the discussions based on graphs (where the reference momenta in the full amplitude $A$ are fixed at once).

In the current paper, we study the single- and double-trace amplitudes with only two negative helicity particles. The helicity configurations can be classified into three categories $\left(g_{i}^{-}, g_{j}^{-}\right),\left(h_{i}^{-}, g_{j}^{-}\right)$and $\left(h_{i}^{-}, h_{j}^{-}\right)$which correspond to EYM amplitudes with (i). two negative-helicity gluons, (ii). one negative-helicity gluon and one negative-helicity graviton and (iii). two negative-helicity gravitons. As stated before, the last configuration has to vanish, while the first two configurations only get contributions from graphs in the MHV sector. In the coming sections, we show that the MHV sectors of single-trace amplitudes with the $\left(g_{i}^{-}, g_{j}^{-}\right)$and $\left(h_{i}^{-}, g_{j}^{-}\right)$configurations precisely match with the corresponding spanning forests of the Hodges determinant form in four dimensions. By generalizing this discussion to double-trace amplitudes, we establish a spanning forest formula for double-trace amplitudes with the $\left(g_{i}^{-}, g_{j}^{-}\right)$configuration. Single-trace amplitudes with the $\left(h_{i}^{-}, h_{j}^{-}\right)$configuration, double-trace amplitudes with $\left(h_{i}^{-}, g_{j}^{-}\right)$and $\left(h_{i}^{-}, h_{j}^{-}\right)$configurations as well as all $m(m>3)$-trace amplitudes with two negative-helicity particles will be proven to vanish.

Before proceeding, we explicitly display the expansions of the single- and double-trace MHV amplitudes by graphs in the corresponding MHV sectors. The general construction rule of the $\mathrm{N}^{k} \mathrm{MHV}$ sector can be found in appendix A. 


\subsection{The expansion of single-trace MHV amplitudes}

Following the above discussion, a single-trace MHV amplitude $A^{(i, j)}(1,2, \ldots, r \| \mathrm{H})$ (where the two negative helicity particles $(i, j)$ can be either $\left(g_{i}, g_{j}\right)$ or $\left.\left(h_{i}, g_{j}\right)\right)$ is given by

$$
A^{(i, j)}(1,2, \ldots, r \| \mathrm{H})=\sum_{\mathcal{F} \in \mathcal{F}[0,1]}(-)^{\mathcal{F}} \mathcal{C}^{\mathcal{F}} A^{(i, j)}(1, \boldsymbol{\sigma}, r)=\sum_{\mathcal{F} \in \mathcal{F}[0,1]} \mathcal{C}^{\mathcal{F}}\left[\sum_{\left.\boldsymbol{\sigma} \in \mathcal{F}\right|_{1} \backslash\{1, r\}} \frac{\langle i, j\rangle^{4}}{(1, \boldsymbol{\sigma}, r)}\right],
$$

in which all graphs $\mathcal{F}$ in the MHV sector for the single-trace amplitude have been summed over, while each pure-YM amplitude $A^{(i, j)}(1, \boldsymbol{\sigma}, r)$ involving two negative-helicity particles $(i, j)$ is further expressed by the Parke-Taylor formula. Here, $(1,2, \ldots, r)$ denotes $\langle 1,2\rangle\langle 2,3\rangle \ldots\langle r, 1\rangle$ for short.

Graphs $\mathcal{F} \in \mathcal{F}[0,1]$ in eq. (2.4) are obtained by the general construction rule in appendix A. To be specific, the general pattern of a graph $\mathcal{F} \in \mathcal{F}[0,1]$ is given by connecting gravitons to gluons in $1 \backslash\{r\}$ via type-2 lines (i.e., $\epsilon \cdot k$ lines) whose arrows point towards the trace $\mathbf{1}$, as shown by figure 2 (a). According to appendix A, the sign $(-)^{\mathcal{F}}$ associating to any graph $\mathcal{F}$ of this pattern is +1 .

\subsection{The expansion of double-trace MHV amplitudes}

Suppose the two gluon traces are $\mathbf{1}=\left\{x_{1}, \ldots, x_{r}\right\}, \mathbf{2}=\left\{y_{1}, \ldots, y_{s}\right\},{ }^{4}$ the graviton set is $\mathbf{H}$ and the two negative-helicity particles are $(i, j)\left((i, j)\right.$ can be either $\left(g_{i}, g_{j}\right)$ or $\left.\left(h_{i}, g_{j}\right)\right)$. A double-trace amplitude $A^{(i, j)}\left(x_{1}, \ldots, x_{r} \mid y_{1}, \ldots, y_{s} \| \mathrm{H}\right)$ is expanded as

$$
A^{(i, j)}\left(x_{1}, \ldots, x_{r} \mid y_{1}, \ldots, y_{s} \| \mathrm{H}\right)=\sum_{\mathcal{F} \in \mathcal{F}[0,2]}(-)^{\mathcal{F}} \mathcal{C}^{\mathcal{F}}\left[\sum_{\boldsymbol{\sigma} \in \mathcal{F}} \sum_{x_{1} \backslash\left\{x_{1}, x_{r}\right\}} \frac{\langle i, j\rangle^{4}}{\left(x_{1}, \boldsymbol{\sigma}, x_{r}\right)}\right],
$$

where we have summed over all possible graphs $\mathcal{F}$ in the MHV sector of double-trace amplitudes.

As stated in appendix $\mathrm{A}$, graphs $\mathcal{F} \in \mathcal{F}[0,2]$ have the general pattern figure 2 (b) where two components $\mathscr{C}_{1}$ and $\mathscr{C}_{2}$, which respectively contains the two traces $\mathbf{1}$ and $\mathbf{2}$, are connected by a type-3 line (i.e. the $k \cdot k$ line). Such a graph has the following pattern:

- Gluons of the trace $\mathbf{1}$ is arranged in the normal order $x_{1}, \ldots, x_{r}$. Assuming the trace $\mathbf{2}$ can be written as $\mathbf{2}=\{a, \boldsymbol{X}, b, \boldsymbol{Y}\}$ where $a, b \in \mathbf{2}$, all gluons in $\mathbf{2}$ are arranged in an order $a, \beta_{1}, \ldots, \beta_{|2|-2}, b$ where $\left\{\beta_{1}, \ldots, \beta_{|2|-2}\right\} \in \boldsymbol{X} \amalg \boldsymbol{Y}^{T}$.

- Each of $\mathscr{C}_{1}$ and $\mathscr{C}_{2}$ in figure 2 (b) is constructed by connecting gravitons therein to gluons in the corresponding trace (except the last element $x_{r}$ in the trace $\mathbf{1}$ ) via type- 2 lines. The arrow of any type- 2 line should point towards the direction of the corresponding trace.

- The two end nodes $x$ and $y$ of the type-3 line $k_{x} \cdot k_{y}$ respectively belong to $\mathscr{C}_{1}$ and $\mathscr{C}_{2}$. The node $x$ satisfies $x \in \mathscr{C}_{1} \backslash\left\{x_{r}\right\}$ and the node $y$ is either the node $a$ or a node which is connected to $a$ via only type-3 lines.

\footnotetext{
${ }^{4}$ Here, we slightly change the labels of gluons so that the two traces are on an equal footing in the final result.
} 


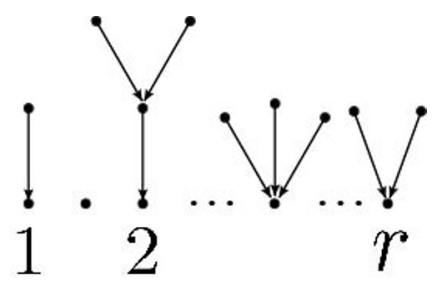

Figure 3. A typical spanning forest for single-trace MHV amplitude. All positive-helicity gravitons and gluons are treated as nodes. All trees in the forest are rooted at gluons.

- The sign $(-)^{\mathcal{F}}$ associating to a graph $\mathcal{F}$ is given by $(-)^{\mathcal{F}} \equiv(-1)^{\mathcal{N}(\mathcal{F})+|2, a, b|}$, where $\mathcal{N}(\mathcal{F})$ is the total number of arrows pointing away from $x_{1},|\mathbf{2}, a, b|$ denotes the number of elements in the $\boldsymbol{Y}$ set if the trace $\mathbf{2}$ can be written as $\mathbf{2}=\{a, \boldsymbol{X}, b, \boldsymbol{Y}\}$ for a given $a \neq b(a \in \mathbf{2})$.

The summation over all graphs $\mathcal{F} \in \mathcal{F}[0,2]$ in eq. (2.5) then means that we should sum over (i). all possible graphs constructed by the above method for a given $a \neq b(b \in \mathbf{2}$ is always fixed) and a given $\beta=\left\{\beta_{1}, \ldots, \beta_{|\mathbf{2}|-2}\right\}$, (ii). all possible $\beta$ for a given $a$, (iii). all possible $a \in \mathbf{2},(a \neq b)$.

\section{Single-trace MHV amplitudes with $\left(g_{i}^{-}, g_{j}^{-}\right)$and $\left(h_{i}^{-}, g_{j}^{-}\right)$}

In this section, we evaluate the single-trace MHV amplitudes $A^{(i, j)}(1,2, \ldots, r \| \mathrm{H})$ with the helicity configurations $\left(g_{i}^{-}, g_{j}^{-}\right)$and $\left(h_{i}^{-}, g_{j}^{-}\right)$by the expansion (2.4). We prove that eq. (2.4) precisely gives the spanning forest form

$$
A^{(i, j)}(1,2, \ldots, r \| \mathrm{H})=(-1)^{N_{h^{-}}} \frac{\langle i, j\rangle^{4}}{(1,2, \ldots, r)}\left[\sum_{\mathcal{G}} \prod_{e(x, y) \in \mathcal{E}(\mathcal{G})} \frac{\langle y, \xi\rangle\langle y, \eta\rangle[y, x]}{\langle x, \xi\rangle\langle x, \eta\rangle\langle y, x\rangle}\right],
$$

which is the spanning forest expansion [27] of the known formula (B.15) (see [25]) that is expressed by Hodges determinant [26]. In the above expression, we summed over all possible spanning forests $\mathcal{G}$ (see figure 3) where all elements in $\{1, \ldots, r\} \cup \mathrm{H}^{+}\left(\mathrm{H}^{+}\right.$is the set of positive-helicity gravitons) were treated as nodes, while the gluons $1, \ldots, r$ were considered as roots. The prefactor $(-1)^{N_{h^{-}}}$is +1 for the $\left(g_{i}^{-}, g_{j}^{-}\right)$configuration and -1 for the $\left(h_{i}^{-}, g_{j}^{-}\right)$configuration. For a given forest $\mathcal{G}, \mathcal{E}(\mathcal{G})$ denotes the set of edges and $e(x, y)$ denotes an edge between nodes $x$ and $y$. For an edge $e(x, y), y$ is always supposed to be nearer to root than $x$ and the edge is dressed by an arrow pointing towards $y$. Each $e(x, y)$ in eq. (3.1) is accompanied by a factor

$$
\frac{\langle y, \xi\rangle\langle y, \eta\rangle[y, x]}{\langle x, \xi\rangle\langle x, \eta\rangle\langle y, x\rangle}
$$

where $\xi$ and $\eta$ are two arbitrarily chosen reference spinors which cannot make the expression divergent. In the following, we prove eq. (3.1) with $\left(g_{i}^{-}, g_{j}^{-}\right)$and $\left(h_{i}^{-}, g_{j}^{-}\right)$configurations in turn. 


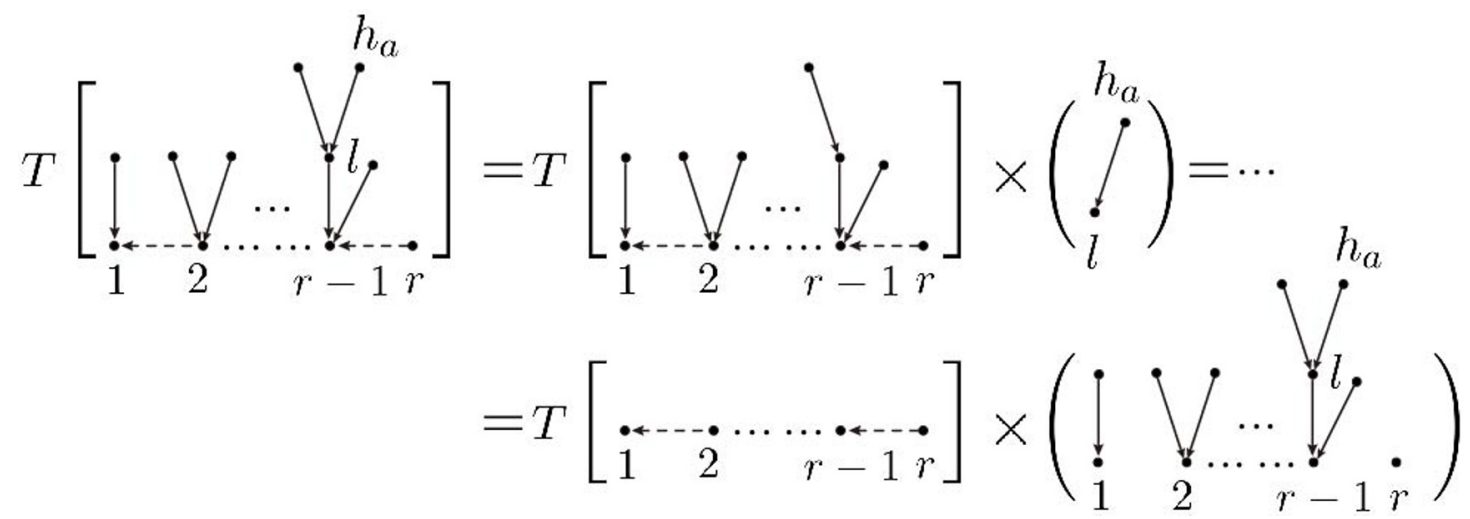

Figure 4. Evaluating a single-trace amplitude with the $\left(g_{i}^{-}, g_{j}^{-}\right)$configuration.

\subsection{Single-trace amplitudes with $\left(g_{i}^{-}, g_{j}^{-}\right)$configuration}

For a single-trace MHV amplitude with the $\left(g_{i}^{-}, g_{j}^{-}\right)$configuration, all gravitons carry positive helicity. As shown in section 2, the reference momenta of all gravitons are taken as the same one, say $\xi^{\mu}$. According to spinor helicity formalism, the Lorentz contraction $\epsilon_{h}^{+} \cdot k_{l}$ between the half polarization $\epsilon_{h}^{+\mu}$ of a graviton $h$ and an arbitrary momentum $k_{l}^{\mu}$ becomes

$$
\epsilon_{h_{a}}^{+}(\xi) \cdot k_{l}=\frac{\langle\xi, l\rangle\left[l, h_{a}\right]}{\left\langle\xi, h_{a}\right\rangle}
$$

Therefore, the coefficient $\mathcal{C}^{\mathcal{F}}$ in eq. (2.4) can be conveniently expressed as

$$
\mathcal{C}^{\mathcal{F}}=\mathcal{C}^{\mathcal{F} \backslash\left\{h_{a}\right\}} \frac{\langle\xi, l\rangle\left[l, h_{a}\right]}{\left\langle\xi, h_{a}\right\rangle}
$$

for a leaf (i.e. an outmost graviton) $h_{a} \in \mathcal{F}$. Here $\mathcal{F} \backslash\left\{h_{a}\right\}$ denotes the subgraph that is obtained from $\mathcal{F}$, by removing $h_{a}$ and the edge attached to it. The node $l$ in eq. (3.4) is supposed to be the neighbour of $h_{a}$. On another hand, the summation over all permutations $\left.\sigma \in \mathcal{F}\right|_{1} \backslash\{1, r\}$ which are established by the graph $\mathcal{F}$ in eq. (2.4) can be achieved by the following steps: (1). summing over all possible permutations $\left.\gamma \in \mathcal{F}\right|_{1} \backslash\left\{1, r, h_{a}\right\}$ established by the graph $\mathcal{F} \backslash\left\{h_{a}\right\} ;(2)$. summing over all possible permutations $\left.\gamma 山\left\{h_{a}\right\}\right|_{l \prec h_{a}}{ }^{5}$ for a given $\gamma$ in the previous step. Thus the summation in square brackets of eq. (2.4) is rearranged into

$$
\sum_{\left.\boldsymbol{\gamma} \in \mathcal{F}\right|_{1} \backslash\left\{1, r, h_{a}\right\}} \sum_{\left.\boldsymbol{\sigma} \in \boldsymbol{\gamma} \uplus\left\{h_{a}\right\}\right|_{l \prec h_{a}}} \frac{\left\langle g_{i}, g_{j}\right\rangle^{4}}{(1, \boldsymbol{\sigma}, r)},
$$

which can be further simplified by the help of the identity (B.8), as follows

$$
\left[\sum_{\left.\boldsymbol{\gamma} \in \mathcal{F}\right|_{1} \backslash\left\{1, r, h_{a}\right\}} \frac{\left\langle g_{i}, g_{j}\right\rangle^{4}}{(1, \gamma, r)}\right] \frac{\langle l, r\rangle}{\left\langle l, h_{a}\right\rangle\left\langle h_{a}, r\right\rangle} .
$$

\footnotetext{
${ }^{5}$ Here we write the constraint condition $\sigma^{-1}(l)<\sigma^{-1}\left(h_{a}\right)$ by $l \prec h_{a}$ for short.
} 
When substituting eq. (3.6) and eq. (3.4) into eq. (2.4) for a given graph $\mathcal{F}$, we arrive

$$
\begin{aligned}
T[\mathcal{F}] & \equiv \mathcal{C}^{\mathcal{F}}\left[\sum_{\left.\boldsymbol{\sigma} \in \mathcal{F}\right|_{1 \backslash\{1, r\}}} \frac{\left\langle g_{i}, g_{j}\right\rangle^{4}}{(1, \boldsymbol{\sigma}, r)}\right] \\
& =\left[\mathcal{C}^{\mathcal{F} \backslash\left\{h_{a}\right\}} \sum_{\left.\gamma \in \mathcal{F}\right|_{1} \backslash\left\{1, r, h_{a}\right\}} \frac{\left\langle g_{i}, g_{j}\right\rangle^{4}}{(1, \gamma, r)}\right] \frac{\langle l, r\rangle\langle l, \xi\rangle\left[l, h_{a}\right]}{\left\langle h_{a}, r\right\rangle\left\langle h_{a}, \xi\right\rangle\left\langle l, h_{a}\right\rangle} \\
& =T\left[\mathcal{F} \backslash\left\{h_{a}\right\}\right] \frac{\langle l, \xi\rangle\langle l, r\rangle\left[l, h_{a}\right]}{\left\langle h_{a}, \xi\right\rangle\left\langle h_{a}, r\right\rangle\left\langle l, h_{a}\right\rangle},
\end{aligned}
$$

where the expression in the square brackets on the second line is just the contribution of the graph $\mathcal{F} \backslash\left\{h_{a}\right\}$. Eq. (3.7) is shown by the first equality of figure 4, while we introduce an arrowed edge $e\left(h_{a}, l\right)$ pointing towards $l$ to denote the factor

$$
\frac{\langle l, \xi\rangle\langle l, r\rangle\left[l, h_{a}\right]}{\left\langle h_{a}, \xi\right\rangle\left\langle h_{a}, r\right\rangle\left\langle l, h_{a}\right\rangle} .
$$

Apparently, the removed line between $h_{a}$ and $l$ in the graph $\mathcal{F}$ is transformed into the edge $e\left(h_{a}, l\right)$ with the new meaning eq. (3.8) in this step.

The equation (3.7) establishes a relation between contributions of a graph $\mathcal{F}$ and the subgraph with one gravion extracted out, hence can be applied iteratively. To be specific, we can further pick out a leaf, say $h_{b}$, from the graph $\mathcal{F} \backslash\left\{h_{a}\right\}$. According to eq. (3.7), $T\left[\mathcal{F} \backslash\left\{h_{a}\right\}\right]$ can be written as

$$
T\left[\mathcal{F} \backslash\left\{h_{a}\right\}\right]=T\left[\mathcal{F} \backslash\left\{h_{a}, h_{b}\right\}\right] \frac{\left\langle l^{\prime}, \xi\right\rangle\left\langle l^{\prime}, r\right\rangle\left[l^{\prime}, h_{b}\right]}{\left\langle h_{b}, \xi\right\rangle\left\langle h_{b}, r\right\rangle\left\langle l^{\prime}, h_{b}\right\rangle},
$$

where $h_{b}$ is supposed to be connected to the node $l^{\prime}$ via a type-2 line. Repeating the above steps until all gravitons have been extracted out, we get the final expression which consists of the following two factors (see the last term in figure 4): (i). the Parke-Taylor factor corresponding to the gluon trace 1, (ii). the coefficient characterized by the forest which has the same structure with that in the original graph $\mathcal{F}$ but with the new meaning of the edges, i.e. (3.8). When all possible graphs $\mathcal{F} \in \mathcal{F}[0,1]$ are summed over, the full single-trace MHV amplitude eq. (2.4) with the $\left(g_{i}^{-}, g_{j}^{-}\right)$configuration is then written as

$$
\frac{\left\langle g_{i}, g_{j}\right\rangle^{4}}{(1,2, \ldots, r)} \sum_{\mathcal{G}^{\prime}} \prod_{e(x, y) \in \mathcal{E}\left(\mathcal{G}^{\prime}\right)} \frac{\langle y, \xi\rangle\langle y, r\rangle[y, x]}{\langle x, \xi\rangle\langle x, r\rangle\langle y, x\rangle} .
$$

In the above expression, we have summed over all possible spanning forests $\mathcal{G}^{\prime}$ where leaves and internal nodes are gravitons, gluons $1,2, \ldots, r-1$ are roots (recalling that in a graph $\mathcal{F}$ (see eq. (2.4)) the last gluon $r$ cannot be connected by a type-2 line). The summation over $\mathcal{G}^{\prime}$ can be further extended to a summation over all forests $\mathcal{G}$ rooted at gluons $1,2, \ldots, r$ because

$$
\frac{\langle r, \xi\rangle\langle r, r\rangle\left[r, h_{a}\right]}{\left\langle h_{a}, \xi\right\rangle\left\langle h_{a}, r\right\rangle\left\langle l, h_{a}\right\rangle}=0 .
$$

Therefore, we get the expression (3.1) with the choice of reference spinor $\eta=r$. Since the r.h.s. of eq. (3.1) can be expressed by the Hodges determinant which is independent of the choice of the reference spinors $\xi$ and $\eta[25,27]$ ), the proof of eq. (3.1) has been completed. 


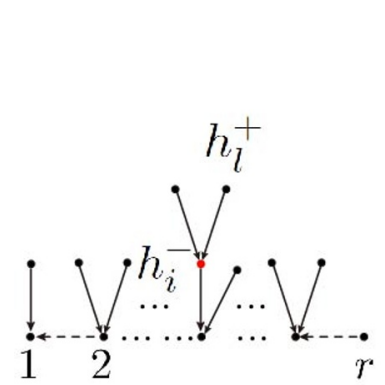

(a)

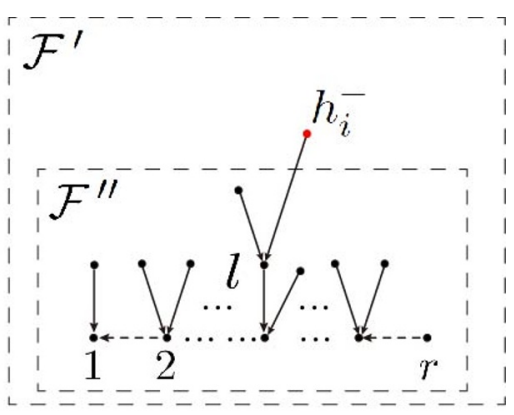

(b)

Figure 5. Typical graphs for the $\left(h_{i}^{-}, g_{j}^{-}\right)$configuration. In (a) the negative-helicity graviton $h_{i}$ is the end node of a type-2 line. In (b), $h_{i}$ plays as a leaf (i.e. an outmost node).

\subsection{Single-trace amplitudes with $\left(h_{i}^{-}, g_{j}^{-}\right)$configuration}

For the $\left(h_{i}^{-}, g_{j}^{-}\right)$configuration, there is one negative-helicity graviton $h_{i}$ and one negativehelicity gluon $g_{j}$. As stated in section 2, the reference momenta of all positive-helicity gravitons are chosen as $k_{h_{i}}^{\mu}$. Consequently,

$$
\epsilon_{h_{l}}^{+}\left(k_{h_{i}}\right) \cdot k_{h_{i}}=0 \text {. }
$$

This indicates those graphs $\mathcal{F}$, where the negative-helicity graviton $h_{i}$ is the ending node of a type-2 line (as shown by figure 5 (a)), have to vanish. In other words, $h_{i}$ in a nonvanishing graph can only be a leaf, as shown by figure 5 (b). Thus the summation over graphs $\mathcal{F} \in \mathcal{F}[0,1]$ in eq. (2.4) becomes a summation $\sum_{\mathcal{F}^{\prime}}$ over all graphs $\mathcal{F}^{\prime}$ with the structure figure 5 (b). This summation can be further arranged as $\sum_{\mathcal{F}^{\prime}} \rightarrow \sum_{\mathcal{F}^{\prime \prime}} \sum_{l \in \mathcal{F}^{\prime \prime} \backslash\{r\}}$, where $\mathcal{F}^{\prime \prime}$ denote those subgraphs (of graphs $\mathcal{F}^{\prime}$ ) which involve only positive-helicity gravitons and all gluons, while $l$ denotes the neighbour of $h_{i}$ in the original graph $\mathcal{F}^{\prime}$ (see figure 5 (b)). Meanwhile, the coefficient $\mathcal{C}^{\mathcal{F}^{\prime}}$ is factorized as

$$
\mathcal{C}^{\mathcal{F}^{\prime}}=\mathcal{C}^{\mathcal{F}^{\prime \prime}}\left(\epsilon_{h_{i}}^{-} \cdot k_{l}\right)=\mathcal{C}^{\mathcal{F}^{\prime \prime}} \frac{[\eta, l]\left\langle l, h_{i}\right\rangle}{\left[\eta, h_{i}\right]},
$$

where $\eta^{\mu}$ is the reference momentum of the negative-helicity graviton $h_{i}$. The permutations $\left.\boldsymbol{\sigma} \in \mathcal{F}^{\prime}\right|_{1} \backslash\{1, r\}$ can be reexpressed as

$$
\left.\boldsymbol{\sigma} \in \boldsymbol{\gamma} \amalg\left\{h_{i}\right\}\right|_{l \prec h_{i}}, \text { where }\left.\boldsymbol{\gamma} \in \mathcal{F}^{\prime \prime}\right|_{1} \backslash\{1, r\} .
$$

Altogether, the single-trace MHV amplitude $A^{\left(h_{i}, g_{j}\right)}(1,2, \ldots, r \| \mathrm{H})$ in eq. (2.4) becomes

$$
\sum_{\mathcal{F}^{\prime \prime}} \mathcal{C}^{\mathcal{F}^{\prime \prime}} \sum_{l \in \mathcal{F}^{\prime \prime} \backslash\{r\}} \frac{[\eta, l]\left\langle l, h_{i}\right\rangle}{\left[\eta, h_{i}\right]}\left[\sum_{\left.\gamma \in \mathcal{F}^{\prime \prime}\right|_{1} \backslash\{1, r\}} \sum_{\left.\boldsymbol{\sigma} \in \boldsymbol{\gamma} \uplus\left\{h_{i}\right\}\right|_{l \prec h_{i}}} \frac{\left\langle h_{i}, g_{j}\right\rangle^{4}}{(1, \boldsymbol{\sigma}, r)}\right] .
$$

When the identity (B.8) is applied, the last summation in the square brackets gives

$$
\sum_{\left.\boldsymbol{\sigma} \in \boldsymbol{\gamma} \uplus\left\{h_{i}\right\}\right|_{l \prec h_{i}}} \frac{\left\langle h_{i}, g_{j}\right\rangle^{4}}{(1, \boldsymbol{\sigma}, r)}=\frac{\left\langle h_{i}, g_{j}\right\rangle^{4}}{(1, \boldsymbol{\gamma}, r)} \frac{\langle l, r\rangle}{\left\langle l, h_{i}\right\rangle\left\langle h_{i}, r\right\rangle},
$$


Here, the first factor is a Parke-Taylor factor which does not involve the graviton $h_{i}$ in the denominator, while the second factor, $\frac{\langle l, r\rangle}{\left\langle l, h_{i}\right\rangle\left\langle h_{i}, r\right\rangle}$, depends on the choice of $l$ and is independent of $\gamma$. Thus the summation of the factors depending on $l$ in eq. (3.15) is given by

$$
\sum_{l \in \mathcal{F}^{\prime \prime} \backslash\{r\}} \frac{[\eta, l]\left\langle l, h_{i}\right\rangle}{\left[\eta, h_{i}\right]} \cdot \frac{\langle l, r\rangle}{\left\langle l, h_{i}\right\rangle\left\langle h_{i}, r\right\rangle}=\sum_{l \in \mathcal{F}^{\prime \prime} \backslash\{r\}} \frac{\langle l, r\rangle[\eta, l]}{\left\langle h_{i}, r\right\rangle\left[\eta, h_{i}\right]}=-\frac{\langle r, r\rangle[\eta, r]}{\left\langle h_{i}, r\right\rangle\left[\eta, h_{i}\right]}-\frac{\left\langle h_{i}, r\right\rangle\left[\eta, h_{i}\right]}{\left\langle h_{i}, r\right\rangle\left[\eta, h_{i}\right]}=-1,
$$

where momentum conservation and the antisymmetry of spinor products have been applied. After this simplification, the amplitude (3.15) turns into

$$
A^{\left(h_{i}, g_{j}\right)}(1,2, \ldots, r \| \mathrm{H})=(-1) \sum_{\mathcal{F}^{\prime \prime}} \mathcal{C}^{\mathcal{F}^{\prime \prime}}\left[\sum_{\left.\gamma \in \mathcal{F}^{\prime \prime}\right|_{1} \backslash\{1, r\}} \frac{\left\langle h_{i}, g_{j}\right\rangle^{4}}{(1, \gamma, r)}\right],
$$

in which, $\mathcal{F}^{\prime \prime}$ are those tree graphs in the MHV sector with the negative-helicity graviton $h_{i}$ deleted. Up to an overall factor, the above expression exactly has the same pattern with the $\left(g_{i}^{-}, g_{j}^{-}\right)$case (i.e. eq. (2.4) with the $\left(g_{i}^{-}, g_{j}^{-}\right)$configuration) in which all gravitons have positive helicity. Thus, following a parallel discussion with the $\left(g_{i}^{-}, g_{j}^{-}\right)$case, we immediately arrive

$$
A^{\left(h_{i}, g_{j}\right)}(1,2, \ldots, r \| \mathrm{H})=(-1) \frac{\left\langle h_{i}, g_{j}\right\rangle^{4}}{(1,2, \ldots, r)}\left[\sum_{\mathcal{G}} \prod_{e(x, y) \in \mathcal{E}(\mathcal{G})} \frac{\left\langle y, h_{i}\right\rangle\langle y, r\rangle[y, x]}{\left\langle x, h_{i}\right\rangle\langle x, r\rangle\langle y, x\rangle}\right],
$$

where we have summed over all spanning forests $\mathcal{G}$ with the node set $\{1, \ldots, r\} \cup \mathrm{H}^{+}$and roots $1, \ldots, r$. This expression is identical with eq. (3.1) when choosing $\xi=h_{i}$ and $\eta=r$. Since eq. (3.1) is independent of the choice of $\xi$ and $\eta[25]$, the proof for the $\left(h_{i}^{-}, g_{j}^{-}\right)$ configuration has been completed.

\subsection{Comments}

Now we summarize some critical features of the above evaluations, which will inspire a symmetric formula of double-trace amplitude with the $\left(g_{i}^{-}, g_{j}^{-}\right)$configuration in the coming section:

- (i). There is a one-to-one correspondence between the refined graphs which contain positive-helicity gravitons and gluons as nodes, and the spanning forests in four dimensions.

- (ii). Although, gravitons are involved in the color-ordered YM amplitudes in the expansion (2.1), they do not appear in the Parke-Taylor factor (except the numerator $\left\langle h_{i}, g_{j}\right\rangle^{4}$ for the $\left(h_{i}^{-}, g_{j}^{-}\right)$configuration) in the spanning forest formula (3.1). Thus the cyclic symmetry of gluons in eq. (3.1) is manifest.

- (iii). The spanning forest formula is independent of the choice of gauge $\xi$ and $\eta$.

In the coming section, we generalize the spanning forest formula (3.1) to double-trace MHV amplitudes with the $\left(g_{i}^{-}, g_{j}^{-}\right)$configuration. 


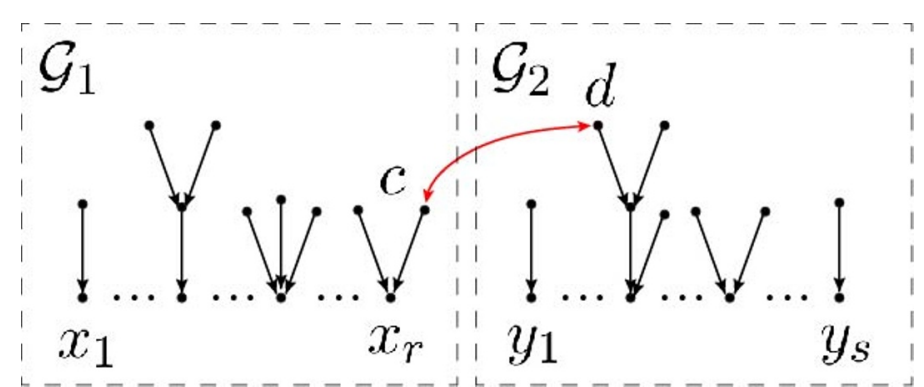

Figure 6. A typical spanning forest for double-trace amplitude with the $\left(g_{i}^{-}, g_{j}^{-}\right)$configuration.

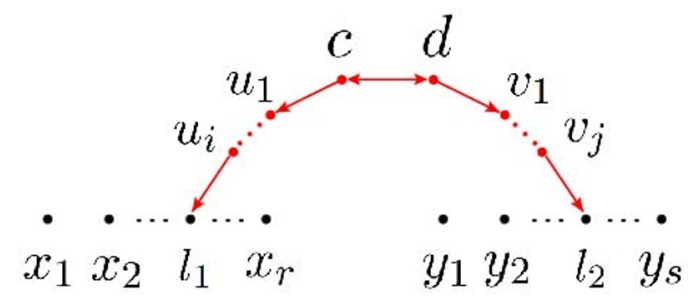

Figure 7. A bridge between the two traces in a spanning forest for a double-trace amplitude with the $\left(g_{i}^{-}, g_{j}^{-}\right)$configuration.

\section{Double-trace MHV amplitudes with $\left(g_{i}^{-}, g_{j}^{-}\right)$configuration}

In this section, we generalize the pattern of single-trace MHV amplitudes (3.1) to doubletrace ones with the $\left(g_{i}^{-}, g_{j}^{-}\right)$configuration. We prove that the double-trace MHV amplitude $A^{\left(g_{i}, g_{j}\right)}\left(x_{1}, \ldots, x_{r} \mid y_{1}, \ldots, y_{s} \| \mathrm{H}\right)$ (where the two gluon traces are $\mathbf{1}=\left\{x_{1}, \ldots, x_{r}\right\}, \mathbf{2}=$ $\left\{y_{1}, \ldots, y_{s}\right\}$ and the graviton set is $\mathbf{H}$ ) satisfies the following spanning forest formula

$$
\begin{aligned}
& A^{\left(g_{i}, g_{j}\right)}\left(x_{1}, \ldots, x_{r} \mid y_{1}, \ldots, y_{s} \| \mathrm{H}\right) \\
& =\frac{\left\langle g_{i}, g_{j}\right\rangle^{4}}{\left(x_{1}, \ldots, x_{r}\right)\left(y_{1}, \ldots, y_{s}\right)}\left[\sum_{\mathcal{G}=\mathcal{G}_{1} \oplus \mathcal{G}_{2}} \sum_{\substack{c \in \mathcal{G}_{1} \\
d \in \mathcal{G}_{2}}}\left(-k_{c} \cdot k_{d}\right) \frac{\langle c, \zeta\rangle\langle d, \chi\rangle}{\langle c, d\rangle\langle\zeta, \chi\rangle} \prod_{e(x, y) \in \mathcal{E}(\mathcal{G})} \frac{\langle\xi, y\rangle\left\langle\lambda_{e}, y\right\rangle[y, x]}{\langle\xi, x\rangle\left\langle\lambda_{e}, x\right\rangle\langle y, x\rangle}\right] .
\end{aligned}
$$

In the above expression, all possible spanning forests $\mathcal{G}$ with the pattern figure 6 are summed over. In each forest $\mathcal{G}$, the node set is given by $\mathbf{1} \cup \mathbf{2} \cup \mathrm{H}$ and all gluons in $\mathbf{1} \cup \mathbf{2}$ are considered as roots. Edges in a forest $\mathcal{G}$ are presented by arrow lines pointing towards roots. Each forest $\mathcal{G}$ is composed of two sub-forests $\mathcal{G}_{1}$ and $\mathcal{G}_{2}$ whose roots live in $\mathbf{1}$ and 2 correspondingly. We further sum over all possible choices of $c \in \mathcal{G}_{1}$ and $d \in \mathcal{G}_{2}$. For a given $\mathcal{G}=\mathcal{G}_{1} \oplus \mathcal{G}_{2}$ and a given choice of $c \in \mathcal{G}_{1}$ and $d \in \mathcal{G}_{2}$, a crossing factor

$$
\left(-k_{c} \cdot k_{d}\right) \frac{\langle c, \zeta\rangle\langle d, \chi\rangle}{\langle c, d\rangle\langle\zeta, \chi\rangle}
$$

is implied where $\zeta$ and $\chi$ are two reference spinors which respectively reflect the cyclic symmetries of the traces $\mathbf{1}$ and $\mathbf{2}$. For convenience, we express this crossing factor by connecting $c$ and $d$ via a double-arrow line, as shown by figure 6 . Any other edge in the graph is either an inner edge or an outer edge, which depends on whether it lives on the 
bridge (see figure 7) between traces 1 and $\mathbf{2}$ or not. Each edge $e(x, y)$ where the arrow points from $x$ to $y$ is associated with a factor

$$
\frac{\langle\xi, y\rangle\left\langle\lambda_{e}, y\right\rangle[y, x]}{\langle\xi, x\rangle\left\langle\lambda_{e}, x\right\rangle\langle y, x\rangle}
$$

where $\xi$ is the common reference spinor of all $e(x, y)$ which reflects the choice of gauge of the half-polarizations in the refined graphs. The $\lambda_{e}$ for each edge $e(x, y)$ is a reference spinor reflecting the cyclic symmetries of the gluon traces. In particular, it is defined by

$$
\lambda_{e}=\left\{\begin{array}{cc}
\eta & (\text { if } e(x, y) \text { is an outer edge ) } \\
\zeta & \left(\text { if } e(x, y) \text { is an inner edge and } e(x, y) \in \mathcal{G}_{1}\right), \\
\chi & \left(\text { if } e(x, y) \text { is an inner edge and } e(x, y) \in \mathcal{G}_{2}\right)
\end{array}\right.
$$

where the reference spinors $\zeta$ and $\chi$ were already introduced before, while $\eta$ is a new reference spinor. It is worth pointing that the summations in the expression (4.1) can be rearranged by (i). first summing over all splits of the graviton set $\mathrm{H} \rightarrow \mathrm{H}_{A}, \mathrm{H}_{B}$; (ii). for a given split, summing over all possible bridges $\mathcal{B}\left(\mathrm{H}_{A}\right)$, which involve all the gravitons in $\mathrm{H}_{A}$ as nodes, between the two traces $\mathbf{1}$ and $\mathbf{2}$, (iii). for a given split and a given bridge, summing over all possible spanning forests $\mathcal{G}^{\prime}$, in which all gravitons and gluons are considered as nodes and all elements in $\mathbf{1} \cup \mathbf{2} \cup \mathrm{H}_{A}$ are considered as roots. The formula (4.1) is then rewritten as an equivalent formula

$$
\begin{aligned}
A^{\left(g_{i}^{-}, g_{j}^{-}\right)}\left(x_{1}, \ldots, x_{r} \mid y_{1}, \ldots, y_{s} \| \mathrm{H}\right) & \frac{\left\langle g_{i}, g_{j}\right\rangle}{4}=\frac{\left\langle y^{\prime}\right.}{\left(x_{1}, \ldots, x_{r}\right)\left(y_{1}, \ldots, y_{s}\right)} \sum_{\mathrm{H}_{\rightarrow} \mathrm{H}_{A}, \mathrm{H}_{B} \mathcal{B}\left(\mathrm{H}_{A}\right)}\left(-k_{c} \cdot k_{d}\right) \frac{\langle c, \zeta\rangle\langle d, \chi\rangle}{\langle c, d\rangle\langle\zeta, \chi\rangle} \prod_{e(x, y) \in \mathcal{E}_{1}\left(\mathcal{B}\left(\mathrm{H}_{A}\right)\right)} \frac{\langle\xi, y\rangle\langle\zeta, y\rangle[y, x]}{\langle\xi, x\rangle\langle\zeta, x\rangle\langle y, x\rangle} \\
& \left.\times \prod_{e(x, y) \in \mathcal{E}_{2}\left(\mathcal{B}\left(\mathrm{H}_{A}\right)\right)} \frac{\langle\xi, y\rangle\langle\chi, y\rangle[y, x]}{\langle\xi, x\rangle\langle\chi, x\rangle\langle y, x\rangle} \sum_{\mathcal{G}^{\prime}} \prod_{e(x, y) \in \mathcal{E}\left(\mathcal{G}^{\prime}\right)} \frac{\langle\xi, y\rangle\langle\eta, y\rangle[y, x]}{\langle\xi, x\rangle\langle\eta, x\rangle\langle y, x\rangle}\right]
\end{aligned}
$$

where $c$ and $d$ are the two gravitons connected by the double arrow line on the bridge $\mathcal{B}\left(\mathrm{H}_{A}\right)$. The $\mathcal{E}_{1}\left(\mathcal{B}\left(\mathrm{H}_{A}\right)\right)$ and $\mathcal{E}_{2}\left(\mathcal{B}\left(\mathrm{H}_{A}\right)\right)$ are the set of single-arrowed edges whose arrows points towards the traces 1 and $\mathbf{2}$, respectively. Graphs $\mathcal{G}^{\prime}$ denote those spanning forests rooted at elements in $\mathbf{1} \cup \mathbf{2} \cup \mathrm{H}_{A}$. Thus all edges of $\mathcal{G}^{\prime}$ are apparently those outer edges in eq. (4.1) and eq. (4.4).

In the following, we first investigate the symmetries of eq. (4.1). After that, we provide an example for eq. (4.1) and then the general proof by the refined graphic rule.

\subsection{Symmetries of the formula}

The spanning forest formula (4.1) is much more symmetric than the expansion (2.5) given by refined graphic rule.

First, permutations in the Parke-Taylor factors of (2.5) involve all external gluons and gravitons. On the contrary, gravitons and gluon traces in (4.1) are disentangled from one another: each trace is expressed by a Parke-Taylor factor with its own gluons in the original permutation, while gravitons and the other trace do not participate in. Therefore, 
eq. (4.1) is explicitly symmetric under the exchanging of the two gluon traces. Moreover, the invariance of eq. (4.1) under exchanging any two gravitons seems more transparent because gravitons are already extracted out from the Parke-Taylor factors and only involved in the summation over spanning forests.

Second, there are "gauge symmetries" corresponding to the arbitrariness of the reference spinors $\xi, \zeta, \chi$ and $\eta$. In the coming subsections, we will see $\xi$ comes from the reference spinor of all 'half' polarizations, thus its arbitrariness is essentially the gauge symmetry of amplitudes. Nevertheless, the arbitrariness of the choices of $\lambda_{e}$ (i.e., $\zeta, \chi$ and $\eta$ ) that encode the cyclic symmetries of the two traces is not so clear. Now let us understand these symmetries of the expression (4.1).

(i). The invariance of (4.1) under the change of $\eta$ This symmetry is easily understood when the amplitude is expressed by eq. (4.5): for a given split $\mathrm{H} \rightarrow \mathrm{H}_{A}, \mathrm{H}_{B}$ and a given bridge between the two traces, the summation over all spanning forests rooted at elements in $\mathbf{1} \cup \mathbf{2} \cup \mathrm{H}_{A}$ exactly produces the determinant of the Hodges matrix (B.16) [25, 27]

$$
\sum_{\mathcal{G}^{\prime}} \prod_{e(x, y) \in \mathcal{E}\left(\mathcal{G}^{\prime}\right)} \frac{\langle\xi, y\rangle\langle\eta, y\rangle[y, x]}{\langle\xi, x\rangle\langle\eta, x\rangle\langle y, x\rangle}=\operatorname{det}\left[\phi_{\mathrm{H}_{B}}\right]
$$

which is independent of the choice of $\eta$. Since each bridge is associated by a Hodges determinant that is independent of $\eta, \eta$ can even be chosen differently for distinct configurations of bridge. Nevertheless, in this paper, we choose all $\eta$ 's corresponding to different bridges as the same one for convenience.

(ii). The invariance of (4.1) under the change of $\zeta$ Since $\eta$ can be chosen arbitrarily, we just set $\eta=\chi$, which does not bring any divergency to eq. (4.1). Now we proceed our discussion by classifying the terms inside the square brackets of eq. (4.1) according to whether $c \in \mathcal{G}_{1}$ is a gluon or a graviton (i.e. $c \in 1$ or $c \in \mathcal{G}_{1} \backslash 1$ ), for given $\mathcal{G}_{1}$ and $\mathcal{G}_{2}$. In the latter case, there exist single-arrowed edges pointing towards $\mathbf{1}$ on the bridge between 1 and $\mathbf{2}$ (see figure 7), while in the former there is no such line on the bridge. The contribution of terms with $c \in 1$ is given by

$$
T_{a}=\sum_{\substack{c \in 1 \\ d \in \mathcal{G}_{2}}}\left(-k_{c} \cdot k_{d}\right) \frac{\langle c, \zeta\rangle\langle d, \chi\rangle}{\langle c, d\rangle\langle\zeta, \chi\rangle}\left[\prod_{e(x, y) \in \mathcal{E}\left(\mathcal{G}=\mathcal{G}_{1} \oplus \mathcal{G}_{2}\right)} \frac{\langle\xi, y\rangle\langle\chi, y\rangle[y, x]}{\langle\xi, x\rangle\langle\chi, x\rangle\langle y, x\rangle}\right]
$$

and the difference between $T_{a}^{\prime}$ and $T_{a}$ corresponding to $\zeta^{\prime}$ and $\zeta$ is evaluated as

$$
\Delta T_{a}=\sum_{\substack{c \in 1 \\ d \in \mathcal{G}_{2}}} \frac{1}{2}[c, d]\langle d, \chi\rangle\langle\chi, c\rangle \frac{\left\langle\zeta^{\prime}, \zeta\right\rangle}{\left\langle\zeta^{\prime}, \chi\right\rangle\langle\zeta, \chi\rangle}\left[\prod_{e(x, y) \in \mathcal{E}\left(\mathcal{G}=\mathcal{G}_{1} \oplus \mathcal{G}_{2}\right)} \frac{\langle\xi, y\rangle\langle\chi, y\rangle[y, x]}{\langle\xi, x\rangle\langle\chi, x\rangle\langle y, x\rangle}\right],
$$


where Schouten identity (B.5) has been applied. On another hand, the expression inside the square brackets of eq. (4.1) when $c \in \mathcal{G}_{1} \backslash \mathbf{1}$ reads

$$
\begin{aligned}
& T_{b}=\sum_{\substack{c \in \mathcal{G}_{1} \backslash 1 \\
d \in \mathcal{G}_{2}}}\left(-k_{c} \cdot k_{d}\right) \frac{\langle c, \zeta\rangle\langle d, \chi\rangle}{\langle c, d\rangle\langle\zeta, \chi\rangle} \\
& \times \prod_{e(x, y) \in \mathcal{E}_{1}(c)} \frac{\langle\xi, y\rangle\langle\zeta, y\rangle[y, x]}{\langle\xi, x\rangle\langle\zeta, x\rangle\langle y, x\rangle} \prod_{\begin{array}{c}
e(x, y) \in \mathcal{E}\left(\mathcal{G}_{1}\right) \\
e(x, y) \notin \mathcal{E}_{1}(c)
\end{array}} \frac{\langle\xi, y\rangle\langle\chi, y\rangle[y, x]}{\langle\xi, x\rangle\langle\chi, x\rangle\langle y, x\rangle} \prod_{e(x, y) \in \mathcal{E}\left(\mathcal{G}_{2}\right)} \frac{\langle\xi, y\rangle\langle\chi, y\rangle[y, x]}{\langle\xi, x\rangle\langle\chi, x\rangle\langle y, x\rangle}
\end{aligned}
$$

where $\mathcal{E}_{1}(c)$ denotes the set of single-arrowed inner edges that are pointing towards the trace $\mathbf{1}$ for a given $c$. Supposing the bridge between the two traces $\mathbf{1}$ and $\mathbf{2}$ is given by figure 7 , the first product in eq. (4.9) for a given $c \in \mathcal{G}_{1} \backslash \mathbf{1}$ and $d \in \mathcal{G}_{2}$ reads

$$
\begin{aligned}
& \prod_{e(x, y) \in \mathcal{E}_{1}(c)} \frac{\langle\xi, y\rangle\langle\zeta, y\rangle[y, x]}{\langle\xi, x\rangle\langle\zeta, x\rangle\langle y, x\rangle} \\
= & \frac{\left\langle\xi, u_{1}\right\rangle\left\langle\zeta, u_{1}\right\rangle\left[u_{1}, c\right]}{\langle\xi, c\rangle\langle\zeta, c\rangle\left\langle u_{1}, c\right\rangle} \frac{\left\langle\xi, u_{2}\right\rangle\left\langle\zeta, u_{2}\right\rangle\left[u_{2}, u_{1}\right]}{\left\langle\xi, u_{1}\right\rangle\left\langle\zeta, u_{1}\right\rangle\left\langle u_{2}, u_{1}\right\rangle} \cdots \frac{\left\langle\xi, l_{1}\right\rangle\left\langle\zeta, l_{1}\right\rangle\left[l_{1}, u_{i}\right]}{\left\langle\xi, u_{i}\right\rangle\left\langle\zeta, u_{i}\right\rangle\left\langle l_{1}, u_{i}\right\rangle} \\
= & {\left[\frac{\left\langle\xi, u_{1}\right\rangle\left\langle\chi, u_{1}\right\rangle\left[u_{1}, c\right]}{\langle\xi, c\rangle\langle\chi, c\rangle\left\langle u_{1}, c\right\rangle} \frac{\left\langle\xi, u_{2}\right\rangle\left\langle\chi, u_{2}\right\rangle\left[u_{2}, u_{1}\right]}{\left\langle\xi, u_{1}\right\rangle\left\langle\chi, u_{1}\right\rangle\left\langle u_{2}, u_{1}\right\rangle} \cdots \frac{\left\langle\xi, l_{1}\right\rangle\left\langle\chi, l_{1}\right\rangle\left[l_{1}, u_{i}\right]}{\left\langle\xi, u_{i}\right\rangle\left\langle\chi, u_{i}\right\rangle\left\langle l_{1}, u_{i}\right\rangle}\right] \frac{\langle\chi, c\rangle}{\left\langle\chi, l_{1}\right\rangle} \frac{\left\langle\zeta, l_{1}\right\rangle}{\langle\zeta, c\rangle} } \\
= & {\left[\prod_{e(x, y) \in \mathcal{E}_{1}(c)} \frac{\langle\xi, y\rangle\langle\chi, y\rangle[y, x]}{\langle\xi, x\rangle\langle\chi, x\rangle\langle y, x\rangle}\right] \frac{\langle\chi, c\rangle}{\left\langle\chi, l_{1}\right\rangle} \frac{\left\langle\zeta, l_{1}\right\rangle}{\langle\zeta, c\rangle} . }
\end{aligned}
$$

The expression (4.9) is therefore arranged as

$$
T_{b}=\left[\sum_{\substack{c \in \mathcal{G}_{1} \backslash \mathbf{1} \\ d \in \mathcal{G}_{2}}} \frac{1}{2}[c, d]\langle\chi, d\rangle \frac{\langle\chi, c\rangle}{\left\langle\chi, l_{1}\right\rangle} \frac{\left\langle\zeta, l_{1}\right\rangle}{\langle\zeta, \chi\rangle}\right]\left[\prod_{e(x, y) \in \mathcal{E}\left(\mathcal{G}=\mathcal{G}_{1} \oplus \mathcal{G}_{2}\right)} \frac{\langle\xi, y\rangle\langle\chi, y\rangle[y, x]}{\langle\xi, x\rangle\langle\chi, x\rangle\langle y, x\rangle}\right]
$$

Apparently, only the first factor involves $\zeta$. When $\zeta$ is replaced by $\zeta^{\prime}$, the first factor differs from the original one (the one with $\zeta$ ) by

$$
\sum_{\substack{c \in \mathcal{G}_{1} \backslash \mathbf{1} \\ d \in \mathcal{G}_{2}}} \frac{1}{2}[c, d]\langle\chi, d\rangle \frac{\langle\chi, c\rangle}{\left\langle\chi, l_{1}\right\rangle}\left(\frac{\left\langle\zeta^{\prime}, l_{1}\right\rangle}{\left\langle\zeta^{\prime}, \chi\right\rangle}-\frac{\left\langle\zeta, l_{1}\right\rangle}{\langle\zeta, \chi\rangle}\right)=\left[\sum_{\substack{c \in \mathcal{G}_{1} \backslash \mathbf{1} \\ d \in \mathcal{G}_{2}}} \frac{1}{2}[c, d]\langle\chi, d\rangle\langle\chi, c\rangle\right] \frac{\left\langle\zeta^{\prime}, \zeta\right\rangle}{\left\langle\chi, \zeta^{\prime}\right\rangle\langle\chi, \zeta\rangle}
$$

where we have applied Schouten identity (B.5). Thus

$$
\Delta T_{b}=\sum_{\substack{c \in \mathcal{G}_{1} \backslash \backslash \\ d \in \mathcal{G}_{2}}} \frac{1}{2}[c, d]\langle d, \chi\rangle\langle\chi, c\rangle \frac{\left\langle\zeta^{\prime}, \zeta\right\rangle}{\left\langle\chi, \zeta^{\prime}\right\rangle\langle\chi, \zeta\rangle}\left[\prod_{e(x, y) \in \mathcal{E}\left(\mathcal{G}=\mathcal{G}_{1} \oplus \mathcal{G}_{2}\right)} \frac{\langle\xi, y\rangle\langle\chi, y\rangle[y, x]}{\langle\xi, x\rangle\langle\chi, x\rangle\langle y, x\rangle}\right]
$$




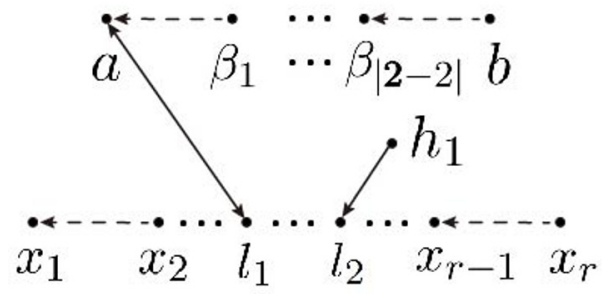

(a)

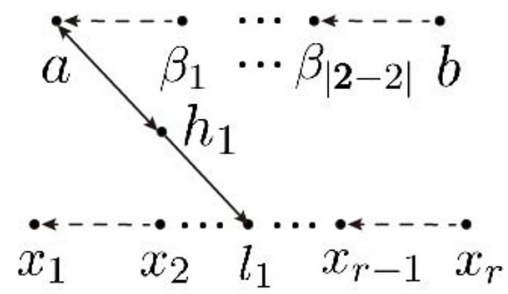

(c)

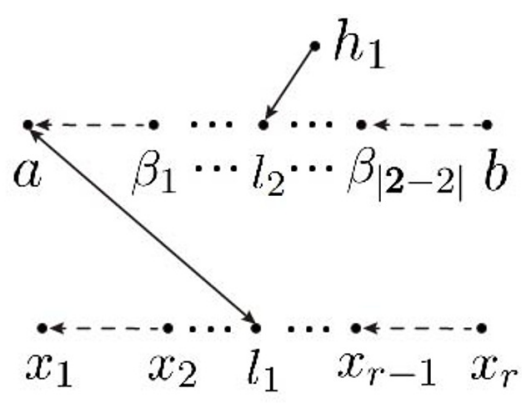

(b)

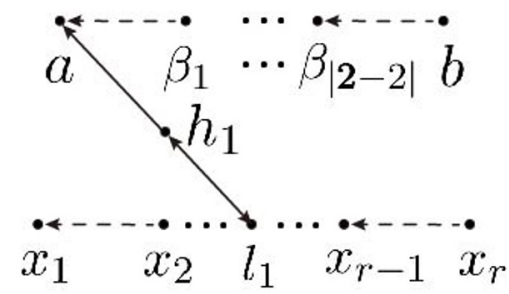

(d)

Figure 8. Typical refined graphs for the double-trace amplitude $A_{m=2}^{\mathrm{MHV}}\left(x_{1}, \ldots, x_{r} \mid y_{1}, \ldots, y_{s} \| h_{1}\right)$.

The sum of $\Delta T_{a}$ and $\Delta T_{b}$ is then given by

$$
\begin{aligned}
& \Delta T_{a}+\Delta T_{b}=\sum_{\substack{c \in \mathcal{G}_{1} \\
d \in \mathcal{G}_{2}}} \frac{1}{2}[c, d]\langle d, \chi\rangle\langle\chi, c\rangle \frac{\left\langle\zeta^{\prime}, \zeta\right\rangle}{\left\langle\chi, \zeta^{\prime}\right\rangle\langle\chi, \zeta\rangle}\left[\prod_{e(x, y) \in \mathcal{E}\left(\mathcal{G}=\mathcal{G}_{1} \oplus \mathcal{G}_{2}\right)} \frac{\langle\xi, y\rangle\langle\chi, y\rangle[y, x]}{\langle\xi, x\rangle\langle\chi, x\rangle\langle y, x\rangle}\right] \\
& =-\sum_{c, d \in \mathcal{G}_{1}} \frac{1}{2}[c, d]\langle d, \chi\rangle\langle\chi, c\rangle \frac{\left\langle\zeta^{\prime}, \zeta\right\rangle}{\left\langle\chi, \zeta^{\prime}\right\rangle\langle\chi, \zeta\rangle}\left[\prod_{e(x, y) \in \mathcal{E}\left(\mathcal{G}=\mathcal{G}_{1} \oplus \mathcal{G}_{2}\right)} \frac{\langle\xi, y\rangle\langle\chi, y\rangle[y, x]}{\langle\xi, x\rangle\langle\chi, x\rangle\langle y, x\rangle}\right], \quad
\end{aligned}
$$

where momentum conservation has been applied. For given $c, d \in \mathcal{G}_{1}$, the last line of the above equation is antisymmetric about $c$ and $d$, thus has to vanish when all nodes $c, d \in \mathcal{G}_{1}$ are summed over. We then conclude that eq. (4.5) (thus eq. (4.1)) is invariant under the change of $\zeta$.

(iii). The invariance of (4.1) under the change of $\chi$ When we choose $\eta=\zeta$, this symmetry naturally follows from a discussion parallel with (ii) because (4.1) has a symmetric form under the exchange of the roles of $\mathbf{1}$ and $\mathbf{2}$.

\subsection{An example study}

Before showing the general proof of the formula (4.1), we explicitly evaluate the doubletrace amplitude $A^{\left(g_{i}^{-}, g_{j}^{-}\right)}\left(x_{1}, \ldots, x_{r} \mid y_{1}, \ldots, y_{s} \| h_{1}\right)$ involving only one graviton. According to the refined graphic rule given in section 2.2, this amplitude can be written as eq. (2.5), in which all typical refined graphs $\mathcal{F} \in \mathcal{F}[0,2]$ are presented by figure 8 . 
We first evaluate the graphs with the structures figure 8 (a) and (b), where the graviton $h_{1}$ plays as an outer node. The contribution of all graphs with the structure figure 8 (a) is given by

$$
\begin{aligned}
T_{1} & =\sum_{\gamma} \sum_{\boldsymbol{\sigma} \in \boldsymbol{\gamma} \amalg\left\{h_{1}\right\}}\left(-k_{a} \cdot k_{l_{1}}\right)\left(\epsilon_{h_{1}} \cdot k_{l_{2}}\right) A\left(x_{1}, \boldsymbol{\sigma}, x_{r}\right) \\
& =\sum_{\gamma}\left(-k_{a} \cdot k_{l_{1}}\right) \sum_{\boldsymbol{\sigma} \in \boldsymbol{\gamma} \amalg\left\{h_{1}\right\}} \frac{\left\langle\xi, l_{2}\right\rangle\left[l_{2}, h_{1}\right]}{\left\langle\xi, h_{1}\right\rangle} \frac{\left\langle g_{i}, g_{j}\right\rangle^{4}}{\left(x_{1}, \boldsymbol{\sigma}, x_{r}\right)} \\
& =\sum_{\gamma}\left(-k_{a} \cdot k_{l_{1}}\right) \frac{\left\langle g_{i}, g_{j}\right\rangle^{4}}{\left(x_{1}, \boldsymbol{\gamma}, x_{r}\right)} \frac{\left\langle\xi, l_{2}\right\rangle\left\langle x_{r}, l_{2}\right\rangle\left[l_{2}, h_{1}\right]}{\left\langle\xi, h_{1}\right\rangle\left\langle x_{r}, h_{1}\right\rangle\left\langle l_{2}, h_{1}\right\rangle} .
\end{aligned}
$$

where $l_{2} \in \mathbf{1} \backslash\left\{x_{r}\right\}=\left\{x_{1}, \ldots, x_{r-1}\right\}$ and the permutations $\gamma$ obeys

$$
\gamma \in\left\{x_{2}, \ldots, l_{1}=x_{a},\left\{x_{a+1}, \ldots, x_{r-1}\right\} ш\left\{a, \beta_{1}, \ldots, \beta_{|\mathbf{2}|-2}, b\right\}\right\} .
$$

On the second line of eq. (4.15), we have written $\epsilon_{h_{1}}(\xi) \cdot k_{l_{2}}$ by spinor products and the MHV Yang-Mills amplitudes $A\left(x_{1}, \boldsymbol{\sigma}, x_{r}\right)$ by Parke-Taylor formula. On the third line of eq. (4.15), the identity (B.8) was applied. Following a similar discussion, the contribution of figure 8 (b) reads

$$
T_{2}=\sum_{\gamma}\left(-k_{a} \cdot k_{l_{1}}\right) \frac{\left\langle g_{i}, g_{j}\right\rangle^{4}}{\left(x_{1}, \gamma, x_{r}\right)} \frac{\left\langle\xi, l_{2}\right\rangle\left\langle x_{r}, l_{2}\right\rangle\left[l_{2}, h_{1}\right]}{\left\langle\xi, h_{1}\right\rangle\left\langle x_{r}, h_{1}\right\rangle\left\langle l_{2}, h_{1}\right\rangle} . \quad\left(l_{2} \in 2\right) .
$$

When summed over all possible $l_{2}$ in $T_{1}$ and $T_{2}$, we arrive the total contribution of graphs with structures figure 8 (a) and (b):

$$
I_{1}=\sum_{l_{2} \in \mathbf{1} \backslash\left\{x_{r}\right\}} T_{1}+\sum_{l_{2} \in \mathbf{2}} T_{2}=\left[\sum_{\gamma}\left(-k_{a} \cdot k_{l_{1}}\right) \frac{\left\langle g_{i}, g_{j}\right\rangle^{4}}{\left(x_{1}, \gamma, x_{r}\right)}\right]\left[\sum_{l_{2} \in \mathbf{1} \cup \mathbf{2}} \frac{\left\langle\xi, l_{2}\right\rangle\left\langle x_{r}, l_{2}\right\rangle\left[l_{2}, h_{1}\right]}{\left\langle\xi, h_{1}\right\rangle\left\langle x_{r}, h_{1}\right\rangle\left\langle l_{2}, h_{1}\right\rangle}\right],
$$

where the case $l_{2}=x_{r}$ is already involved in the second equality since $\left\langle x_{r}, x_{r}\right\rangle=0$. The first factor in the above expression is explicitly written as

$$
\begin{aligned}
& \sum_{\gamma}\left(-k_{a} \cdot k_{l_{1}}\right) \frac{\left\langle g_{i}, g_{j}\right\rangle^{4}}{\left(x_{1}, \ldots, l_{1}=x_{a}, \boldsymbol{\gamma} \in\left\{x_{a+1}, \ldots, x_{r-1}\right\} \amalg\{a, \boldsymbol{\beta}, b\}, x_{r}\right)} \\
& =\left(-k_{a} \cdot k_{l_{1}}\right) \frac{\left\langle g_{i}, g_{j}\right\rangle^{4}}{\left(x_{1}, \ldots, x_{r}\right)} \frac{\left\langle l_{1}, x_{r}\right\rangle}{\left\langle l_{1}, a, \boldsymbol{\beta}, b, x_{r}\right\rangle} \\
& =\frac{\left\langle g_{i}, g_{j}\right\rangle^{4}}{\left(x_{1}, \ldots, x_{r}\right)} \frac{1}{(a, \boldsymbol{\beta}, b)}\left(-k_{a} \cdot k_{l_{1}}\right) \frac{\langle b, a\rangle\left\langle l_{1}, x_{r}\right\rangle}{\left\langle l_{1}, a\right\rangle\left\langle b, x_{r}\right\rangle},
\end{aligned}
$$

in which, the identity (B.9) has been applied on the second line. When all possible $\beta$ (with the sign $(-1)^{|\mathbf{2}, a, b|}$ ) are summed over, the factor $\frac{1}{(a, \boldsymbol{\beta}, b)}$ turns into $\frac{1}{\left(y_{1}, \ldots, y_{s}\right)}$, according to the Kleiss-Kuijf (KK) relation [36] (B.13) between Parke-Taylor factors. Therefore all contributions of graphs with the structures figure 8 (a) and (b) are collected as

$$
I_{1}=\frac{\left\langle g_{i}, g_{j}\right\rangle^{4}}{\left(x_{1}, \ldots, x_{r}\right)\left(y_{1}, \ldots, y_{s}\right)}\left[\sum_{l_{1} \in \mathbf{1}, a \in \mathbf{2}}\left(-k_{a} \cdot k_{l_{1}}\right) \frac{\langle a, b\rangle\left\langle l_{1}, x_{r}\right\rangle}{\left\langle l_{1}, a\right\rangle\left\langle x_{r}, b\right\rangle}\right]\left[\sum_{l_{2} \in \mathbf{1} \cup \mathbf{2}} \frac{\left\langle\xi, l_{2}\right\rangle\left\langle x_{r}, l_{2}\right\rangle\left[l_{2}, h_{1}\right]}{\left\langle\xi, h_{1}\right\rangle\left\langle x_{r}, h_{1}\right\rangle\left\langle l_{2}, h_{1}\right\rangle}\right] .
$$


Each graph with the structure figure 8 (c) contributes

$$
T_{3}=\left(-k_{a} \cdot k_{h_{1}}\right)\left(\epsilon_{h_{1}} \cdot k_{l_{1}}\right) \sum_{\sigma} \frac{\left\langle g_{i}, g_{j}\right\rangle^{4}}{\left(x_{1}, x_{2}, \ldots, l_{1}=x_{a}, \boldsymbol{\sigma} \in\left\{x_{a+1}, \ldots, x_{r-1}\right\} \amalg\left\{h_{1}, a, \beta, b\right\}, x_{r}\right)} .
$$

Noting that permutation $\boldsymbol{\sigma}$ in each Parke-Taylor factor can be rewritten as follows

$$
\left.\boldsymbol{\sigma} \in\left\{x_{a+1}, \ldots, x_{r-1}\right\} \uplus\left\{h_{1}\right\} \amalg\{a, \boldsymbol{\beta}, b\}\right|_{h_{1} \prec a},
$$

we are able to apply the identity (B.9) to extract the trace $\mathbf{2}$ and the graviton $h_{1}$ from the Parke-Taylor factor in turn. The $T_{3}$ is then expressed as

$$
\begin{aligned}
T_{3} & =\left(-k_{a} \cdot k_{h_{1}}\right)\left(\epsilon_{h_{1}} \cdot k_{l_{1}}\right) \sum_{\gamma} \frac{\left\langle g_{i}, g_{j}\right\rangle^{4}}{\left(x_{1}, \ldots, l_{1}=x_{a}, \boldsymbol{\gamma} \in\left\{x_{a+1}, \ldots, x_{r-1}\right\} ш\left\{h_{1}\right\}, x_{r}\right)} \frac{1}{(a, \beta, b)} \frac{\left\langle h_{1}, x_{r}\right\rangle\langle b, a\rangle}{\left\langle h_{1}, a\right\rangle\left\langle b, x_{r}\right\rangle} \\
& =\frac{\left\langle g_{i}, g_{j}\right\rangle^{4}}{\left(x_{1}, \ldots, x_{r}\right)} \frac{1}{(a, \beta, b)}\left[\left(-k_{a} \cdot k_{h_{1}}\right) \frac{\left\langle h_{1}, x_{r}\right\rangle\langle b, a\rangle}{\left\langle h_{1}, a\right\rangle\left\langle b, x_{r}\right\rangle}\right]\left[\frac{\left\langle\xi, l_{1}\right\rangle\left\langle x_{r}, l_{1}\right\rangle\left[l_{1}, h_{1}\right]}{\left\langle\xi, h_{1}\right\rangle\left\langle x_{r}, h_{1}\right\rangle\left\langle l_{1}, h_{1}\right\rangle}\right] .
\end{aligned}
$$

When all possible $\boldsymbol{\beta}$ (with the sign $(-1)^{|\mathbf{2}, a, b|}$ ), $a \in \mathbf{2} \backslash\{b\}, l_{1} \in \mathbf{1} \backslash\left\{x_{r}\right\}$ are summed over and the KK relation [36] is applied to the trace $\mathbf{2}$, the contribution of all graphs with the structure figure 8 (c) becomes

$$
I_{2}=\frac{\left\langle g_{i}, g_{j}\right\rangle^{4}}{\left(x_{1}, \ldots, x_{r}\right)\left(y_{1}, \ldots, y_{s}\right)}\left[\sum_{a \in \mathbf{2}}\left(-k_{a} \cdot k_{h_{1}}\right) \frac{\left\langle h_{1}, x_{r}\right\rangle\langle b, a\rangle}{\left\langle h_{1}, a\right\rangle\left\langle b, x_{r}\right\rangle}\right]\left[\sum_{l_{1} \in \mathbf{1}} \frac{\left\langle\xi, l_{1}\right\rangle\left\langle x_{r}, l_{1}\right\rangle\left[l_{1}, h_{1}\right]}{\left\langle\xi, h_{1}\right\rangle\left\langle x_{r}, h_{1}\right\rangle\left\langle l_{1}, h_{1}\right\rangle}\right] .
$$

Here, the terms with $a=b$ and $l_{1}=x_{r}$ were also included in the corresponding summations since $\langle b, b\rangle=\left\langle x_{r}, x_{r}\right\rangle=0$.

Each graph with the structure figure 8 (d) provides a contribution

$T_{4}=\left(-k_{h_{1}} \cdot k_{l_{1}}\right)\left(-\epsilon_{h_{1}} \cdot k_{a}\right) \sum_{\gamma} \frac{\left\langle g_{i}, g_{j}\right\rangle^{4}}{\left(x_{1}, x_{2}, \ldots, l_{1}=x_{a}, \boldsymbol{\gamma} \in\left\{x_{a+1}, \ldots, x_{r-1}\right\} \amalg\left\{h_{1}, a, \boldsymbol{\beta}, b\right\}, x_{r}\right)}$.

When the identity (B.9) is applied, $T_{4}$ turns into

$$
\begin{aligned}
T_{4} & =\left(-k_{h_{1}} \cdot k_{l_{1}}\right)\left(-\epsilon_{h_{1}} \cdot k_{a}\right) \frac{\left\langle g_{i}, g_{j}\right\rangle^{4}}{\left(x_{1}, \ldots, x_{r}\right)} \frac{\left\langle l_{1}, x_{r}\right\rangle}{\left\langle l_{1}, h_{1}, a, \beta, b, x_{r}\right\rangle} \\
& =\left(-\epsilon_{h_{1}} \cdot k_{a}\right) \frac{\left\langle g_{i}, g_{j}\right\rangle^{4}}{\left(x_{1}, \ldots, x_{r}\right)} \frac{1}{\left(h_{1}, a, \beta, b\right)}\left[\left(-k_{h_{1}} \cdot k_{l_{1}}\right) \frac{\left\langle l_{1}, x_{r}\right\rangle\left\langle b, h_{1}\right\rangle}{\left\langle l_{1}, h_{1}\right\rangle\left\langle b, x_{r}\right\rangle}\right],
\end{aligned}
$$

where $\left\langle a_{1}, a_{2}, \ldots, a_{i}\right\rangle \equiv\left\langle a_{1}, a_{2}\right\rangle\left\langle a_{2}, a_{3}\right\rangle \ldots\left\langle a_{i-1}, a_{i}\right\rangle$. We further apply the identity (B.8) to extract $h_{1}$ from the trace 2 (i.e. the Parke-Taylor factor $\left.1 /\left(h_{1}, a, \boldsymbol{\beta}, b\right)\right)$ and write $\epsilon_{h_{1}} \cdot k_{a}$ by spinor products, then obtain

$$
T_{4}=\frac{\left\langle g_{i}, g_{j}\right\rangle^{4}}{\left(x_{1}, \ldots, x_{r}\right)} \frac{1}{(a, \beta, b)}\left[\left(-k_{h_{1}} \cdot k_{l_{1}}\right) \frac{\left\langle l_{1}, x_{r}\right\rangle\left\langle b, h_{1}\right\rangle}{\left\langle l_{1}, h_{1}\right\rangle\left\langle b, x_{r}\right\rangle}\right]\left[\frac{\langle\xi, a\rangle\langle b, a\rangle\left[a, h_{1}\right]}{\left\langle\xi, h_{1}\right\rangle\left\langle b, h_{1}\right\rangle\left\langle a, h_{1}\right\rangle}\right] .
$$




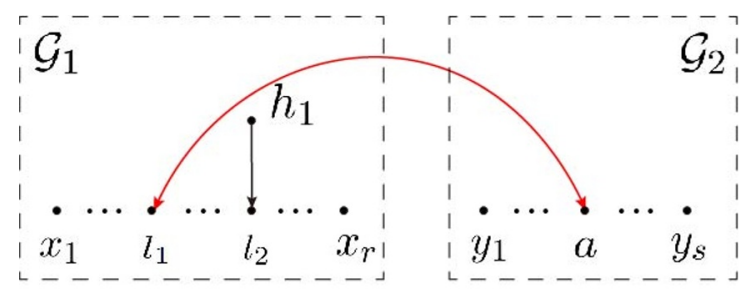

(a)

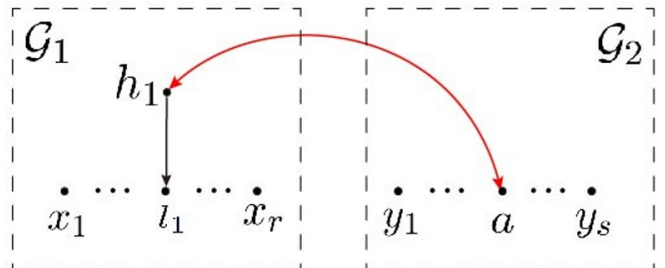

(c)

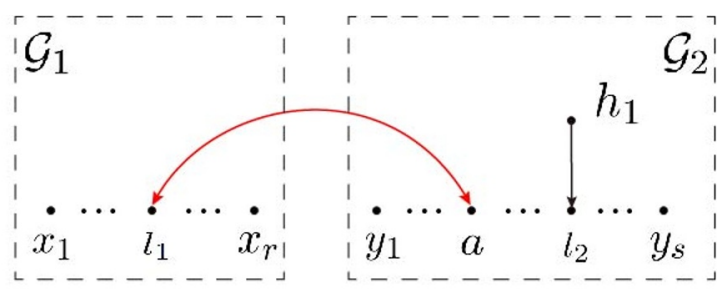

(b)

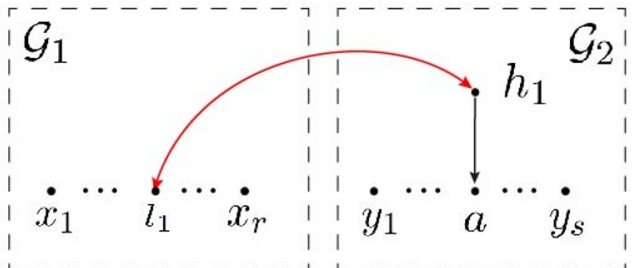

(d)

Figure 9. Spanning forests for the double-trace amplitude $A^{\left(g_{i}, g_{j}\right)}\left(x_{1}, \ldots, x_{r} \mid y_{1}, \ldots, y_{s} \| h_{1}\right)$.

Summing over all $\boldsymbol{\beta} \in \mathrm{KK}[\mathbf{2}, a, b]$ (with the sign $\left.(-1)^{|\mathbf{2}, a, b|}\right), a \in \mathbf{2} \backslash\{b\}, l_{1} \in \mathbf{1} \backslash\left\{x_{r}\right\}$ and applying the KK relation on the Parke-Taylor factors of trace 2 , we get

$$
I_{3}=\frac{\left\langle g_{i}, g_{j}\right\rangle^{4}}{\left(x_{1}, \ldots, x_{r}\right)\left(y_{1}, \ldots, y_{s}\right)}\left[\sum_{l_{1} \in \mathbf{1}}\left(-k_{h_{1}} \cdot k_{l_{1}}\right) \frac{\left\langle l_{1}, x_{r}\right\rangle\left\langle b, h_{1}\right\rangle}{\left\langle l_{1}, h_{1}\right\rangle\left\langle b, x_{r}\right\rangle}\right]\left[\sum_{a \in \mathbf{2}} \frac{\langle\xi, a\rangle\langle b, a\rangle\left[a, h_{1}\right]}{\left\langle\xi, h_{1}\right\rangle\left\langle b, h_{1}\right\rangle\left\langle a, h_{1}\right\rangle}\right],
$$

where $a=b$ and $l_{1}=x_{r}$ are included again due to the antisymmetry of the spinor products.

The contribution of all refined graphs with the structures figure 8 are obtained by summing $I_{1}, I_{2}$ and $I_{3}$ together:

$$
\begin{aligned}
A^{\left(g_{i}, g_{j}\right)}\left(x_{1}, \ldots, x_{r} \mid y_{1}, \ldots, y_{s} \| h_{1}\right) \\
=\frac{\left\langle g_{i}, g_{j}\right\rangle}{\left(x_{1}, \ldots, x_{r}\right)\left(y_{1}, \ldots, y_{s}\right)} \\
\quad \times\left[\sum_{l_{1} \in \mathbf{1}, a \in \mathbf{2}}\left(-k_{a} \cdot k_{l_{1}}\right) \frac{\left\langle l_{1}, x_{r}\right\rangle\langle a, b\rangle}{\left\langle l_{1}, a\right\rangle\left\langle x_{r}, b\right\rangle} \sum_{l_{2} \in \mathbf{1} \cup \mathbf{2}} \frac{\left\langle\xi, l_{2}\right\rangle\left\langle l_{2}, x_{r}\right\rangle\left[l_{2}, h_{1}\right]}{\left\langle\xi, h_{1}\right\rangle\left\langle h_{1}, x_{r}\right\rangle\left\langle l_{2}, h_{1}\right\rangle}\right. \\
\quad+\sum_{a \in \mathbf{2}}\left(-k_{a} \cdot k_{h_{1}}\right) \frac{\left\langle h_{1}, x_{r}\right\rangle\langle a, b\rangle}{\left\langle h_{1}, a\right\rangle\left\langle x_{r}, b\right\rangle} \sum_{l_{1} \in \mathbf{1}} \frac{\left\langle\xi, l_{1}\right\rangle\left\langle l_{1}, x_{r}\right\rangle\left[l_{1}, h_{1}\right]}{\left\langle\xi, h_{1}\right\rangle\left\langle h_{1}, x_{r}\right\rangle\left\langle l_{1}, h_{1}\right\rangle} \\
\left.\quad+\sum_{l_{1} \in \mathbf{1}}\left(-k_{h_{1}} \cdot k_{l_{1}}\right) \frac{\left\langle l_{1}, x_{r}\right\rangle\left\langle h_{1}, b\right\rangle}{\left\langle l_{1}, h_{1}\right\rangle\left\langle x_{r}, b\right\rangle} \sum_{a \in \mathbf{2}} \frac{\langle\xi, a\rangle\langle a, b\rangle\left[a, h_{1}\right]}{\left\langle\xi, h_{1}\right\rangle\left\langle h_{1}, b\right\rangle\left\langle a, h_{1}\right\rangle}\right] .
\end{aligned}
$$

which precisely reproduces the formula (4.1) with the choice of gauge $\zeta=x_{r}$ and $\chi=b$. In this example, all possible graphs expressed by the spanning forests in eq. (4.1) are given by 

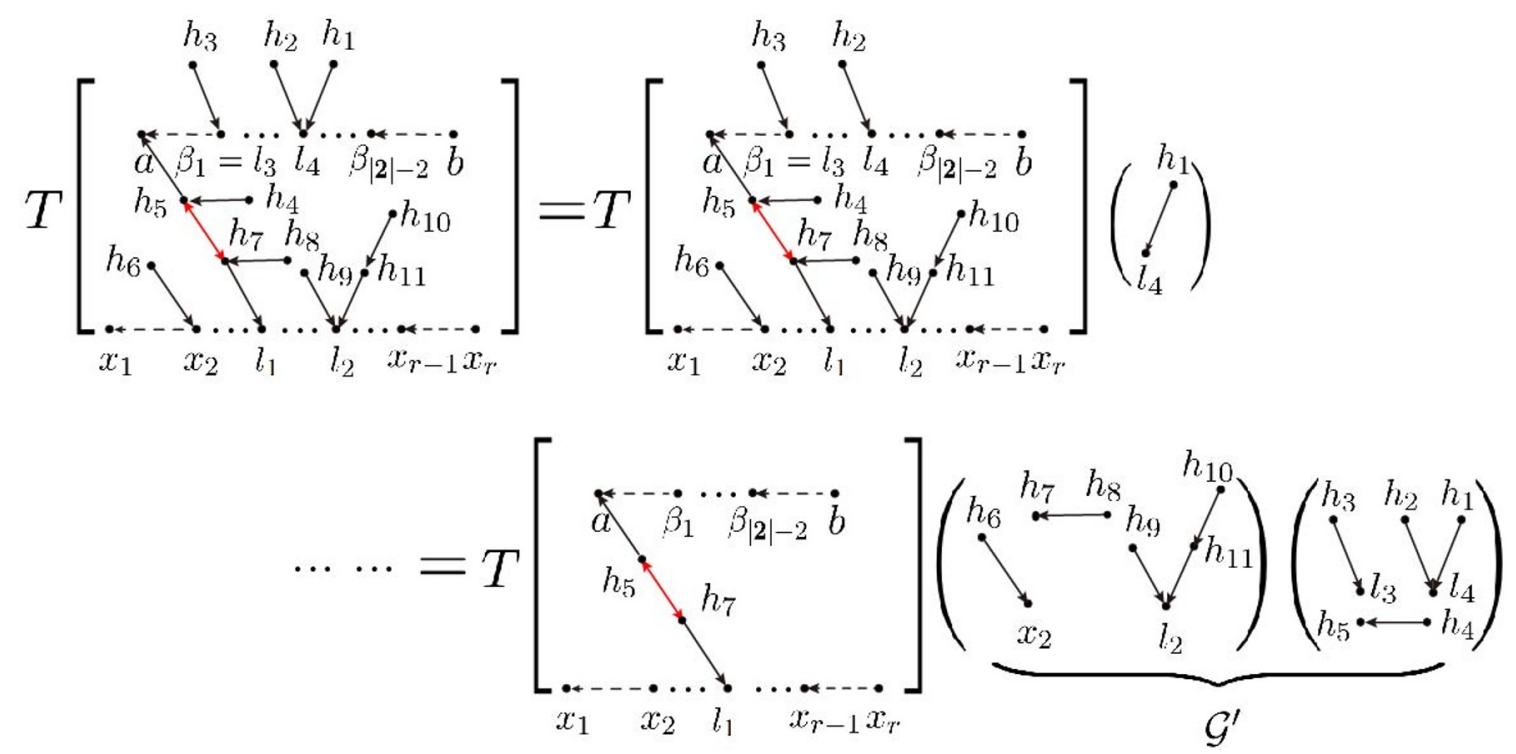

Figure 10. An example for step-1 in the general proof of the formula (4.1). Here, the permutation $\beta$ satisfies $\beta \in \mathrm{KK}[\mathbf{2}, a, b]$. The graviton sets $\left\{h_{1}, h_{2}, h_{3}\right\},\left\{h_{4}, h_{8}\right\}$ and $\left\{h_{6}, h_{9}, h_{10}, h_{11}\right\}$ are the sets of outer gravitons which belong to those trees planted at gluons in the trace 2 (i.e. $l_{3}$ and $l_{4}$ ), gluons in the trace 1 (i.e. $l_{1}$ and $l_{2}$ ) and the inner gravitons (i.e. $h_{5}$ and $h_{7}$ ), respectively. After manipulations in this step, only the two gluon traces as well as the inner gravitons $h_{5}, h_{7}$ remain in the refined graph. All other gravitons (and the tree structures associated to them) have been extracted out and described by the spanning forest $\mathcal{G}^{\prime}$.

figure 9, where both figure 9 (a) and (b) contribute to the first term, figure 9 (c) and (d) correspond to the second and the third term. The cyclic symmetry of the traces demand that the above expression must be preserved when we consider any other gluons $x_{u} \in 1$ and $y_{v} \in \mathbf{2}$ as the last ones instead of $x_{r}$ and $b$ respectively. This has already been guaranteed by the symmetry under choosing different $\zeta$ and $\chi$ in (4.1).

\subsection{The general proof}

Inspired by the above example and the study of single-trace amplitudes, we now prove the general formula (4.1) by three steps: step-1. extracting all outer gravitons from the Parke-Taylor factors in eq. (2.5), step-2. separating the two traces, which may be attached by inner gravitons, from one another, step-3. extracting the inner gravitons from the corresponding Parke-Taylor factor. The explicit proof is following.

Step-1. For a given refined graph $\mathcal{F}$ in eq. (2.5), we pick a leaf (i.e. an outermost graviton) $h_{a}$. Permutations established by $\mathcal{F}$ can be written as $\left\{x_{1},\left(\left.\mathcal{F}\right|_{x_{1}} \backslash\left\{x_{1}, h_{a}, x_{r}\right\}\right) ш\right.$ $\left.\left.\left\{h_{a}\right\}\right|_{l \prec h_{a}}, x_{r}\right\}$, where $l$ is supposed to be the node adjacent to $h_{a}$. The coefficient corresponding to the graph $\mathcal{F}$ then reads

$$
\mathcal{C}^{\mathcal{F}}=\mathcal{C}^{\mathcal{F} \backslash\left\{h_{a}\right\}}\left(\epsilon_{h_{a}} \cdot k_{l}\right) .
$$


The YM amplitudes in eq. (2.5) corresponding to the graph $\mathcal{F}$ are collected as

$$
\begin{aligned}
\sum_{\left.\boldsymbol{\sigma} \in \mathcal{F}\right|_{x_{1}} \backslash\left\{x_{1}, x_{r}\right\}} \frac{\left\langle g_{i}, g_{j}\right\rangle^{4}}{\left(x_{1}, \boldsymbol{\sigma}, x_{r}\right)} & =\sum_{\gamma \in \mathcal{F}||_{x_{1}} \backslash\left\{x_{1}, h_{a}, x_{r}\right\}} \sum_{\left.\boldsymbol{\sigma} \in\left(\boldsymbol{\gamma} \uplus\left\{h_{a}\right\}\right)\right|_{l \prec h_{a}}} \frac{\left\langle g_{i}, g_{j}\right\rangle^{4}}{\left(x_{1}, \boldsymbol{\sigma}, x_{r}\right)} \\
& =\sum_{\left.\gamma \in \mathcal{F}\right|_{x_{1}} \backslash\left\{x_{1}, h_{a}, x_{r}\right\}} \frac{\left\langle g_{i}, g_{j}\right\rangle^{4}}{\left(x_{1}, \gamma, x_{r}\right)} \frac{\left\langle l, x_{r}\right\rangle}{\left\langle l, h_{a}\right\rangle\left\langle h_{a}, x_{r}\right\rangle},
\end{aligned}
$$

where the identity (B.8) has been applied. Hence the total contribution of the graph $\mathcal{F}$ is recursively expressed as

$$
\begin{aligned}
T^{\mathcal{F}} & =\left[\begin{array}{ll}
\mathcal{C}^{\mathcal{F} \backslash\left\{h_{a}\right\}} & \sum_{\gamma \in \mathcal{F} \mid x_{1} \backslash\left\{x_{1}, h_{a}, x_{r}\right\}} \frac{\left\langle g_{i}, g_{j}\right\rangle^{4}}{\left(x_{1}, \gamma, x_{r}\right)}
\end{array}\right]\left[\left(\epsilon_{h_{a}} \cdot k_{l}\right) \frac{\left\langle l, x_{r}\right\rangle}{\left\langle l, h_{a}\right\rangle\left\langle h_{a}, x_{r}\right\rangle}\right] \\
& =\left[\begin{array}{ll}
\mathcal{C}^{\mathcal{F} \backslash\left\{h_{a}\right\}} & \sum_{\left.\gamma \in \mathcal{F}\right|_{x_{1}} \backslash\left\{x_{1}, h_{a}, x_{r}\right\}} \frac{\left\langle g_{i}, g_{j}\right\rangle^{4}}{\left(x_{1}, \gamma, x_{r}\right)}
\end{array}\right] \frac{\langle\xi, l\rangle\left\langle x_{r}, l\right\rangle\left[l, h_{a}\right]}{\left\langle\xi, h_{a}\right\rangle\left\langle x_{r}, h_{a}\right\rangle\left\langle l, h_{a}\right\rangle} \\
& =T^{\mathcal{F} \backslash\left\{h_{a}\right\}} \frac{\langle\xi, l\rangle\left\langle x_{r}, l\right\rangle\left[l, h_{a}\right]}{\left\langle\xi, h_{a}\right\rangle\left\langle x_{r}, h_{a}\right\rangle\left\langle l, h_{a}\right\rangle} .
\end{aligned}
$$

On the second line, the explicit expression of $\epsilon_{h_{a}} \cdot k_{l}$ has been substituted and the expression in the square brackets is nothing but the contribution of the graph when the node $h_{a}$ is deleted. Applying the above relation iteratively until all elements in the outer-graviton set $\mathrm{H}_{B}$ have been removed from the graph $\mathcal{F}$, we arrive

$$
T^{\mathcal{F}}=T^{\mathcal{F} \backslash \mathrm{H}_{B}} \prod_{e\left(h_{a}, l\right) \in \mathcal{E}\left(\mathcal{G}^{\prime}\right)} \frac{\langle\xi, l\rangle\left\langle x_{r}, l\right\rangle\left[l, h_{a}\right]}{\left\langle\xi, h_{a}\right\rangle\left\langle x_{r}, h_{a}\right\rangle\left\langle l, h_{a}\right\rangle},
$$

where $\mathcal{E}\left(\mathcal{G}^{\prime}\right)$ denotes the set of edges belonging to the spanning forest $\mathcal{G}^{\prime}$ that is the collection of trees planted at nodes in $\left(\mathbf{1} \backslash\left\{x_{r}\right\}\right) \cup \mathbf{2} \cup \mathrm{H}_{A}$. These trees in $\mathcal{G}^{\prime}$ have the same structures with those in the corresponding refined graph $\mathcal{F}$, but the edges have the new meaning. After this step, the remaining refined graph $\mathcal{F} \backslash \mathrm{H}_{B}$ involves only the two traces and the inner gravitons (i.e. gravitons on the bridge between the two traces). Manipulations in this step is shown by figure 10 .

Step-2. Now we separate the two traces $\mathbf{1}$ and $\mathbf{2}$ (and the inner gravitons attached to each) from one another. Assume that the bridge between $\mathbf{1}$ and $\mathbf{2}$ in the remaining refined graph has the following structure

$$
l_{1}=x_{k} \in \mathbf{1} \backslash\left\{x_{r}\right\} \leftarrow c_{1} \leftarrow \cdots \leftarrow c_{u-1} \leftarrow c\left(=c_{u}\right) \leftrightarrow d\left(=d_{1}\right) \rightarrow \cdots \rightarrow d_{v} \rightarrow a \in \mathbf{2} \backslash\{b\},
$$

where each type- 2 line is expressed by $\leftarrow$ or $\rightarrow$, the type- 3 line is denoted by $\leftrightarrow$, the gluon $l_{1} \in \mathbf{1}$ is supposed to be $x_{k}$. According to the refined graphic rule, $T^{\mathcal{F} \backslash \mathrm{H}_{B}}$ in eq. (4.33) is explicitly written as

$$
\begin{aligned}
T^{\mathcal{F} \backslash \mathrm{H}_{B}}= & {\left[\sum_{\uplus} \frac{\left\langle g_{i}, g_{j}\right\rangle^{4}}{\left(x_{1}, \ldots, x_{k},\left\{x_{k+1}, \ldots, x_{r-1}\right\} \amalg\left\{c_{1}, \ldots, c=c_{u}, d=d_{1}, \ldots, d_{v}, a, \beta, b\right\}, x_{r}\right)}\right] } \\
& \times\left(-k_{c} \cdot k_{d}\right) \prod_{p=1}^{u} \epsilon_{c_{p}} \cdot k_{c_{p-1}} \prod_{q=1}^{v}\left(-\epsilon_{d_{q}} \cdot k_{d_{q+1}}\right)
\end{aligned}
$$




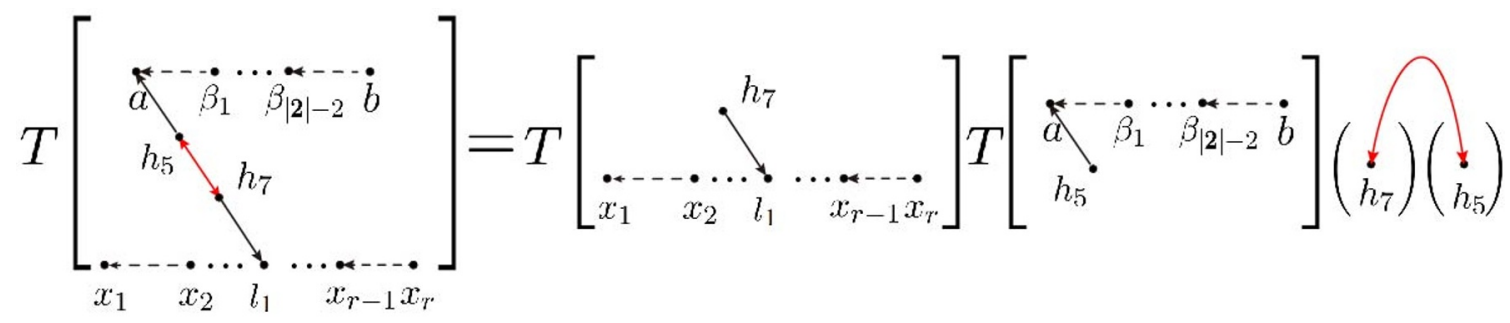

Figure 11. An example for step-2 in the general proof of the formula (4.1). In this step, the contribution of the remaining refined graph in figure 10 is further decomposed such that the two traces and the inner gravitons attached to them are separated. After this step, the double-arrow line between $h_{5}$ and $h_{7}$ now stands for the crossing factor $\left(-k_{h_{5}} \cdot k_{h_{7}}\right) \frac{\left\langle h_{5}, b\right\rangle\left\langle x_{r}, h_{7}\right\rangle}{\left\langle h_{7}, h_{5}\right\rangle\left\langle b, x_{r}\right\rangle}$.

where $c_{0} \equiv x_{k}$ and $d_{v+1} \equiv a$. Note that the permutations $\left\{x_{k+1}, \ldots, x_{r-1}\right\} \sqcup\left\{c_{1}, \ldots, c=\right.$ $\left.c_{u}, d=d_{1}, \ldots, d_{v}, a, \beta, b\right\}$ can be reexpressed as

$$
\left.\left(\gamma \in\left\{x_{k+1}, \ldots, x_{r-1}\right\} \amalg\left\{c_{1}, \ldots, c=c_{u}\right\}\right) \amalg\left\{d=d_{1}, \ldots, d_{v}, a, \beta, b\right\}\right|_{c \prec d} .
$$

When the identity (B.9) is applied, the expression in the square brackets of eq. (4.35) turns into

$$
\begin{aligned}
& \sum_{\gamma} \frac{\left\langle g_{i}, g_{j}\right\rangle^{4}}{\left(x_{1}, \ldots, x_{k}, \gamma, x_{r}\right)} \frac{\left\langle c, x_{r}\right\rangle}{\left\langle c, d=d_{1}, \ldots, d_{v}, a, \beta, b, x_{r}\right\rangle} \\
& =\sum_{\gamma} \frac{\left\langle g_{i}, g_{j}\right\rangle^{4}}{\left(x_{1}, \ldots, x_{k}, \gamma, x_{r}\right)\left(d=d_{1}, \ldots, d_{v}, a, \beta, b\right)} \frac{\langle b, d\rangle\left\langle c, x_{r}\right\rangle}{\langle c, d\rangle\left\langle b, x_{r}\right\rangle}
\end{aligned}
$$

Thus the contribution of two traces (and inner gravitons attached to them) in eq. (4.35) are separated as follows

$$
\begin{aligned}
T^{\mathcal{F} \backslash \mathbf{H}_{B}=} & \left(-k_{c} \cdot k_{d}\right) \frac{\left\langle c, x_{r}\right\rangle\langle d, b\rangle}{\langle c, d\rangle\left\langle x_{r}, b\right\rangle} \\
& \times\left[\sum_{\gamma} \frac{\left\langle g_{i}, g_{j}\right\rangle^{4}}{\left(x_{1}, \ldots, x_{k}, \gamma, x_{r}\right)} \prod_{p=1}^{u} \epsilon_{c_{p}} \cdot k_{c_{p-1}}\right]\left[\frac{1}{\left(d=d_{1}, \ldots, d_{v}, a, \beta, b\right)} \prod_{q=1}^{v}\left(-\epsilon_{d_{q}} \cdot k_{d_{q+1}}\right)\right] .
\end{aligned}
$$

Here, without loss of generality, the factor $\left\langle g_{i}, g_{j}\right\rangle^{4}$ is assumed to be absorbed into the Parke-Taylor factor involving the trace 1 . This step is demonstrated by figure 11 .

Step-3. We now extract the gravitons $c_{1}, \ldots, c_{u}$ and $d_{1}, \ldots, d_{v}$ from the traces 1 and $\mathbf{2}$ respectively. The contribution of the trace $\mathbf{1}$ (and the inner gravitons attached to it) is given by the expression inside the first pair of square brackets in eq. (4.38), which is further 


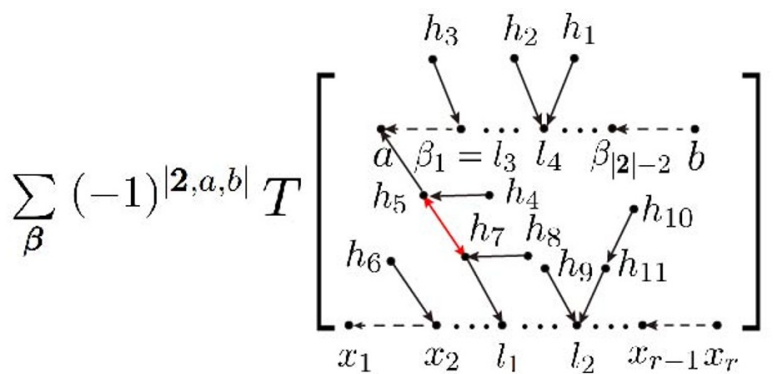

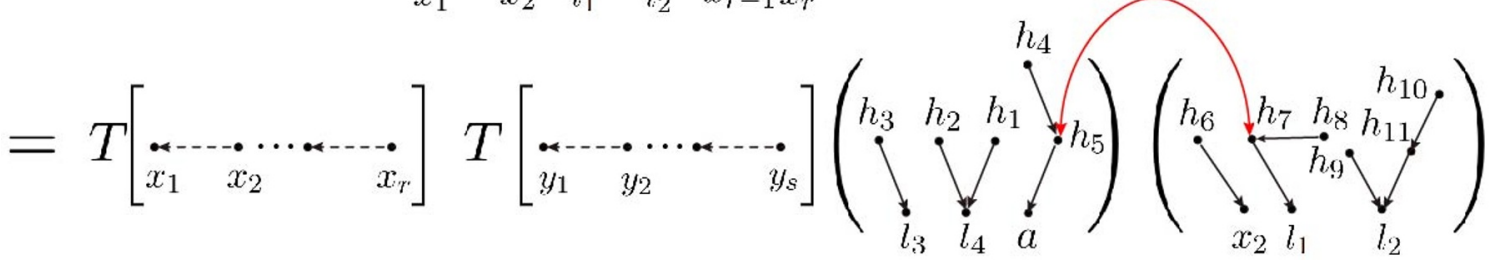

Figure 12. An example for step-3 in the general proof of the formula (4.1). In this step, the inner gravitons $h_{7}$ and $h_{5}$ in figure 12 are further extracted from the traces $\mathbf{1}$ and $\mathbf{2}$ respectively. After summing over all $\beta \in \mathrm{KK}[\mathbf{2}, a, b]$ with the proper sign $(-1)^{|\mathbf{2}, a, b|}$, the second trace becomes a ParkeTaylor factor with the standard permutation $y_{1}, y_{2}, \ldots, y_{s}$. Therefore, we get the structure (4.42). Here the gluons in $\mathbf{1}$ and $\mathbf{2}$ are considered as roots, while gravitons $h_{5}$ and $h_{7}$ play as the inner gravitons. The bridge between the two traces is $l_{1} \leftarrow h_{7} \leftrightarrow h_{5} \rightarrow a$.

simplified as

$$
\begin{gathered}
\sum_{\gamma} \frac{\left\langle g_{i}, g_{j}\right\rangle^{4}}{\left(x_{1}, \ldots, x_{k}, \boldsymbol{\gamma}, x_{r}\right)} \prod_{p=1}^{u} \epsilon_{c_{p}} \cdot k_{c_{p-1}} \\
=\frac{\left\langle g_{i}, g_{j}\right\rangle^{4}}{\left(x_{1}, \ldots, x_{r}\right)}\left(\prod_{p=1}^{u} \frac{\left\langle c_{p-1}, x_{r}\right\rangle}{\left\langle c_{p-1}, c_{p}\right\rangle\left\langle c_{p}, x_{r}\right\rangle}\right)\left(\prod_{p=1}^{u} \epsilon_{c_{p}} \cdot k_{c_{p-1}}\right) \\
=\frac{\left\langle g_{i}, g_{j}\right\rangle^{4}}{\left(x_{1}, \ldots, x_{r}\right)} \prod_{p=1}^{u} \frac{\left\langle\xi, c_{p-1}\right\rangle\left\langle x_{r}, c_{p-1}\right\rangle\left[c_{p-1}, c_{p}\right]}{\left\langle\xi, c_{p}\right\rangle\left\langle x_{r}, c_{p}\right\rangle\left\langle c_{p-1}, c_{p}\right\rangle},
\end{gathered}
$$

where permutations $\boldsymbol{\gamma}$ satisfy $\gamma \in\left\{x_{k+1}, \ldots, x_{r-1}\right\} \amalg\left\{c_{1}, \ldots, c=c_{u}\right\}$. For a given $c_{p}$ in eq. (4.39), the identity (B.8) has been applied and the explicit expression of $\epsilon_{c_{p}} \cdot k_{c_{p-1}}$ has been inserted. For the trace 2 in eq. (4.38), we extract gravitons $d_{1}, \ldots, d_{v}$ out from the corresponding Parke-Taylor factor (noting that the Parke-Taylor factor has cyclic symmetry) as follows

$$
\begin{gathered}
\frac{1}{\left(d=d_{1}, \ldots, d_{v}, a, \beta, b\right)} \prod_{q=1}^{v}\left(-\epsilon_{d_{q}} \cdot k_{d_{q+1}}\right) \\
=\frac{1}{(a, \beta, b)}\left(\prod_{q=1}^{v} \frac{\left\langle b, d_{q+1}\right\rangle}{\left\langle b, d_{q}\right\rangle\left\langle d_{q}, d_{q+1}\right\rangle}\right) \prod_{q=1}^{v}\left(-\epsilon_{d_{q}} \cdot k_{d_{q+1}}\right) \\
=\frac{1}{(a, \boldsymbol{\beta}, b)} \prod_{q=1}^{v} \frac{\left\langle\xi, d_{q+1}\right\rangle\left\langle b, d_{q+1}\right\rangle\left[d_{q+1}, d_{q}\right]}{\left\langle\xi, d_{q}\right\rangle\left\langle b, d_{q}\right\rangle\left\langle d_{q+1}, d_{q}\right\rangle} .
\end{gathered}
$$

When we sum over all possible permutations $\beta \in \mathrm{KK}[2, a, b]$, each of which is dressed by the sign $(-1)^{|2, a, b|}$, the Parke-Taylor factors $1 \backslash(a, \beta, b)$ turn into the one with the standard 
permutation $y_{1}, \ldots, y_{s}$, because of the KK relation (B.13). We thus get

$$
\frac{1}{\left(y_{1}, \ldots, y_{s}\right)} \prod_{q=1}^{v} \frac{\left\langle\xi, d_{q+1}\right\rangle\left\langle b, d_{q+1}\right\rangle\left[d_{q+1}, d_{q}\right]}{\left\langle\xi, d_{q}\right\rangle\left\langle b, d_{q}\right\rangle\left\langle d_{q+1}, d_{q}\right\rangle} .
$$

Eq. (4.38), eq. (4.39) and eq. (4.41) together imply that eq. (4.33) can be reexpressed as (shown by figure 12)

$$
\begin{array}{r}
\frac{\left\langle g_{i}, g_{j}\right\rangle^{4}}{\left(x_{1}, \ldots, x_{r}\right)\left(y_{1}, \ldots, y_{s}\right)}\left(-k_{c} \cdot k_{d}\right) \frac{\left\langle c, x_{r}\right\rangle\langle d, b\rangle}{\langle c, d\rangle\left\langle x_{r}, b\right\rangle} \prod_{p=1}^{u} \frac{\left\langle\xi, c_{p-1}\right\rangle\left\langle x_{r}, c_{p-1}\right\rangle\left[c_{p-1}, c_{p}\right]}{\left\langle\xi, c_{p}\right\rangle\left\langle x_{r}, c_{p}\right\rangle\left\langle c_{p-1}, c_{p}\right\rangle} \\
\times \prod_{q=1}^{v} \frac{\left\langle\xi, d_{q+1}\right\rangle\left\langle b, d_{q+1}\right\rangle\left[d_{q+1}, d_{q}\right]}{\left\langle\xi, d_{q}\right\rangle\left\langle b, d_{q}\right\rangle\left\langle d_{q+1}, d_{q}\right\rangle} \prod_{e\left(h_{a}, l\right) \in \mathcal{E}\left(\mathcal{G}^{\prime}\right)} \frac{\langle\xi, l\rangle\left\langle x_{r}, l\right\rangle\left[l, h_{a}\right]}{\left\langle\xi, h_{a}\right\rangle\left\langle x_{r}, h_{a}\right\rangle\left\langle l, h_{a}\right\rangle}
\end{array}
$$

when all possible $\boldsymbol{\beta} \in \mathrm{KK}[\mathbf{2}, a, b]$ are summed over. This is exactly one term of eq. (4.5), while the inner graviton set is $\mathrm{H}_{A}=\left\{c_{1}, \cdots, c_{u}, d_{1}, \cdots, d_{v}\right\}$, the bridge $\mathcal{B}\left(H_{A}\right)$ between the two traces is given by (4.34) and the choice of gauge is

$$
\chi=b, \quad \zeta=\eta=x_{r} .
$$

After summing over all splits $\mathrm{H} \rightarrow \mathrm{H}_{A}, \mathrm{H}_{B}$, all possible bridges (4.34) for a given split $\mathrm{H} \rightarrow \mathrm{H}_{A}, \mathrm{H}_{B}$ and all spanning forests rooted at $\left(\mathbf{1} \backslash\left\{x_{r}\right\}\right) \cup \mathbf{2} \cup \mathrm{H}_{A}$ for a given bridge, we finally get eq. (4.5) (thus eq. (4.1)) with the choice of gauge (4.43) (noting that the terms in eq. (4.5) which invlove trees planted on $x_{r}$ and/or the bridge (4.34) with $a=b$ must vanish under this choice of gauge, due to the antisymmetry of spinor products). Since we have already proven that eq. (4.5) is independent of the choice of gauge, the general proof of eq. (4.5) (thus eq. (4.1)) has been completed.

\section{The vanishing configurations}

In this section, we investigate the vanishing amplitudes with two negative-helicity particles. We first introduce several properties of refined graphs when the corresponding $Y M$ amplitudes in the expansion (2.1) are MHV amplitudes (which are expressed by ParkeTaylor formula). Using these properties, we prove that the double-trace amplitudes with the $\left(h_{i}^{-}, g_{j}^{-}\right)$configuration, the single- and double- trace amplitudes with the $\left(h_{i}^{-}, h_{j}^{-}\right)$configuration as well as all amplitudes with arbitrary two negative-helicity particles and more than two traces have to vanish.

\subsection{Helpful properties of refined graphs for amplitudes with two negative- helicity particles}

Now we present several usful properties of the refined graphs when the amplitude has two negative-helicity particles. Our choice of gauge is always the standard one: i.e. all the reference momenta of positive-helicity gravitons are chosen as $k_{h_{i}}^{\mu}$ ( $h_{i}$ is a negative-helicity graviton), while the reference momenta of all negative-helicity gravitons, i.e. the $h_{i}$ for the $\left(h_{i}^{-}, g_{j}^{-}\right)$configuration and $h_{i}, h_{j}$ for the $\left(h_{i}^{-}, h_{j}^{-}\right)$configuration, are chosen as $\xi^{\mu}$. With this choice of gauge, the refined graphs satisfy the following properties. 


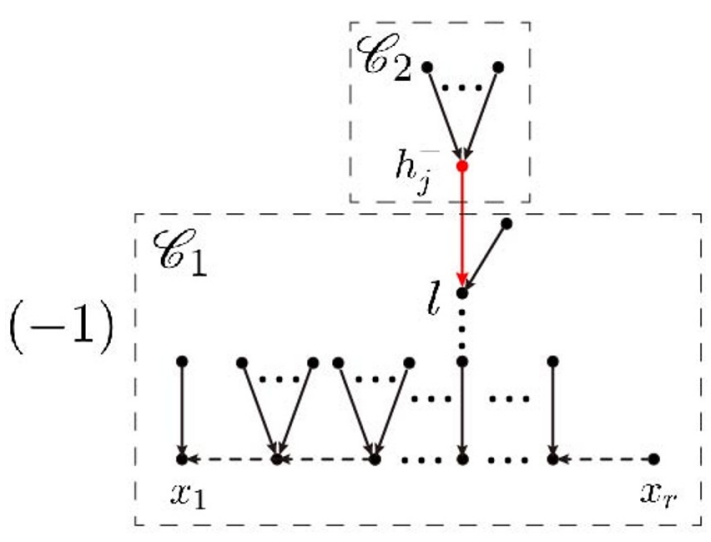

(a)

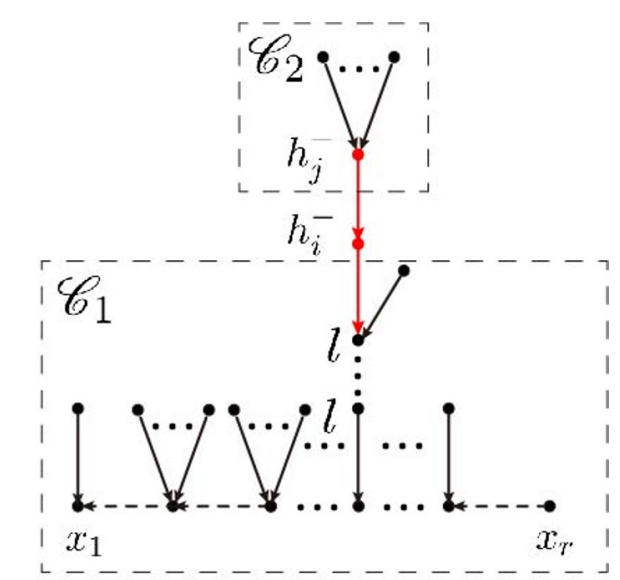

(b)

Figure 13. Graphs with the structures (a) and (b) cancel with each other if the corresponding amplitudes are described by Parke-Taylor factors.

Property-1. Suppose $h_{i}$ is a negative-helicity graviton and $\left\{\mathcal{F}^{\prime}\right\}$ is the set of graphs satisfying the following two conditions: (i). The $h_{i}$ is a leaf which starts a type-2 line $\epsilon_{h_{i}} \cdot k_{l}$, (ii). All graphs in $\left\{\mathcal{F}^{\prime}\right\}$ are reduced to an identical graph $\mathcal{F}$ when $h_{i}$ is deleted. The sum of contributions of all graphs in $\left\{\mathcal{F}^{\prime}\right\}$ is reduced to the contribution of $\mathcal{F}=\mathcal{F}^{\prime} \backslash\left\{h_{a}\right\}$ with an extra minus. This property, when the graphs $\mathcal{F}^{\prime}$ involve only one component (see figure 5), has already been proven in section 3.2. Proof for the cases with more general structure follows from a similar statement.

Property-2. Graphs of the patterns figure 13 (a) and (b) must cancel with each other. This is shown as follows. When we extract the gravitons from the Parke-Taylor factors, the contributions of $\mathscr{C}_{1}\left(\mathscr{C}_{2}\right)$ are the same for distinct graphs figure 13 (a) and (b). Thus we only need to compare the contribution of the substructures colored red. In figure 13 (a), the red part contributes a factor

$$
(-1)\left(\epsilon_{h_{j}}^{-} \cdot k_{l}\right)\left(\frac{\left\langle l, x_{r}\right\rangle}{\left\langle l, h_{j}\right\rangle\left\langle h_{j}, x_{r}\right\rangle}\right)
$$

where the first factor comes from the definition of the type-2 line and the second factor is obtained when extracting $h_{j}$ out from the Parke-Taylor factor, $(-1)$ is the overall sign in figure 13 (a). When the first factor is expressed by spinor products, the above expression is reduced into

$$
-\frac{\left\langle l, h_{j}\right\rangle[\xi, l]}{\left[\xi, h_{j}\right]} \frac{\left\langle l, x_{r}\right\rangle}{\left\langle l, h_{j}\right\rangle\left\langle h_{j}, x_{r}\right\rangle}=-\frac{\left\langle l, x_{r}\right\rangle[\xi, l]}{\left\langle h_{j}, x_{r}\right\rangle\left[\xi, h_{j}\right]} .
$$




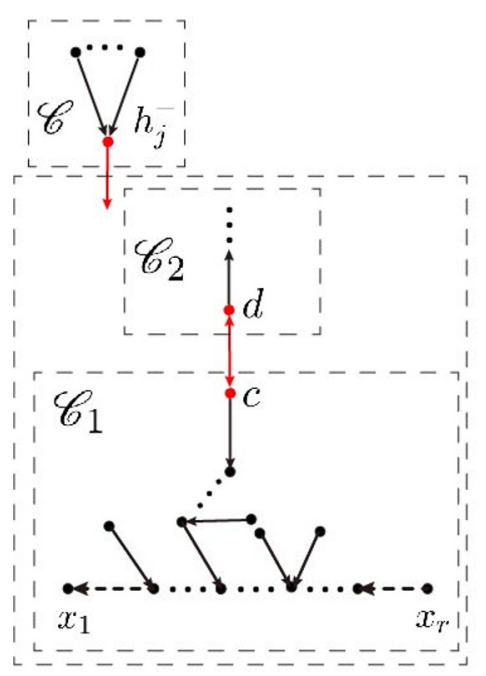

(a)

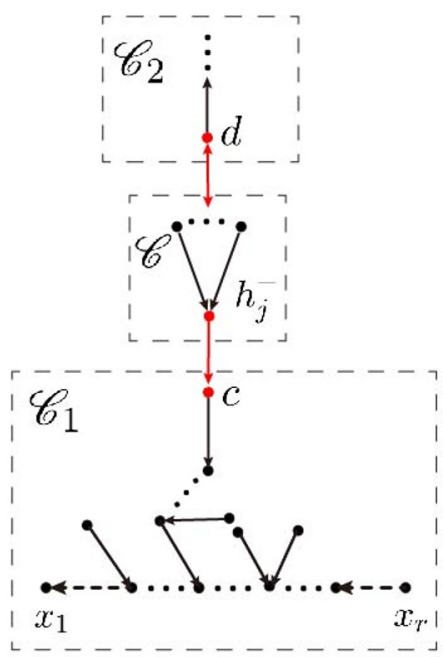

(b)

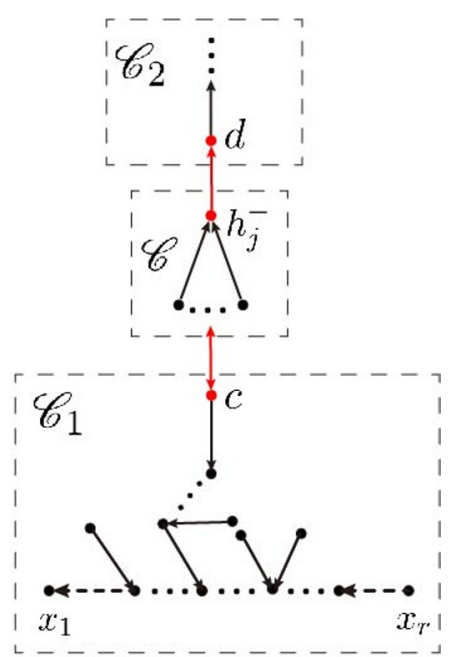

(c)

Figure 14. Contributions of graphs with the typical structures (a), (b) and (c) cancel with each other.

The red part of figure 13 (b) contributes the following factor

$$
\begin{aligned}
\left(\epsilon_{h_{j}}^{-} \cdot k_{h_{i}}\right) & \frac{\left\langle h_{i}, x_{r}\right\rangle}{\left\langle h_{i}, h_{j}\right\rangle\left\langle h_{j}, x_{r}\right\rangle}\left(\epsilon_{h_{i}}^{-} \cdot k_{l}\right) \frac{\left\langle l, x_{r}\right\rangle}{\left\langle l, h_{i}\right\rangle\left\langle h_{i}, x_{r}\right\rangle} \\
& =\frac{\left\langle h_{i}, h_{j}\right\rangle\left[\xi, h_{i}\right]}{\left[\xi, h_{j}\right]} \frac{\left\langle h_{i}, x_{r}\right\rangle}{\left\langle h_{i}, h_{j}\right\rangle\left\langle h_{j}, x_{r}\right\rangle} \frac{\left\langle l, h_{i}\right\rangle[\xi, l]}{\left[\xi, h_{i}\right]} \frac{\left\langle l, x_{r}\right\rangle}{\left\langle l, h_{i}\right\rangle\left\langle h_{i}, x_{r}\right\rangle} \\
& =\frac{\left\langle l, x_{r}\right\rangle[\xi, l]}{\left\langle h_{j}, x_{r}\right\rangle\left[\xi, h_{j}\right]},
\end{aligned}
$$

which is just the corresponding factor in figure 13 (a) with an opposite sign. Thus figure 13 (a) and (b) cancel with each other.

Property-3. Let $\mathscr{C}$ be a tree structure where all lines are type-2 lines whose arrows point towards the negative-helicity graviton $h_{j}, \mathscr{C}_{1}$ and $\mathscr{C}_{2}$ be other two tree structures. Suppose the trace $\mathbf{1}=\left\{x_{1}, \ldots, x_{r}\right\}$ belongs to $\mathscr{C}_{1}$. Graphs with the structures figure 14 (a), (b) and (c) must cancel with each other. In figure 14, a line connected to a boxed region but not a concrete node always means we sum over graphs where the line is connected to all nodes (except $x_{r} \in 1$ ) in that region. To prove the cancellation between these three graphs, we only need to consider the $\mathscr{C}$ part and the red lines in each graph because either the $\mathscr{C}_{1}$ or the $\mathscr{C}_{2}$ part provides an identical expression in all three graphs.

- (a). In figure 14 (a), when extracting the $\mathscr{C}$ part from the Parke-Taylor factors we get a factor of the following form

$$
\left[\left(\epsilon_{h_{j}} \cdot k_{l}\right) \frac{\left\langle l, x_{r}\right\rangle}{\left\langle l, h_{j}\right\rangle\left\langle h_{j}, x_{r}\right\rangle}\right]\left[\prod_{e(x, y) \in \mathcal{E}(\mathscr{C})}\left(\epsilon_{x} \cdot k_{y}\right) \frac{\left\langle y, x_{r}\right\rangle}{\langle y, x\rangle\left\langle x, x_{r}\right\rangle}\right],
$$


where $l \in\left(\mathscr{C}_{1} \backslash\left\{x_{r}\right\}\right) \cup \mathscr{C}_{2}$ is supposed to be the end node of the type-2 line which starts at $h_{j}$. We extract the node $d$ after extracting all other nodes in $\mathscr{C}_{2}$ part from the Parke-Taylor factors. Then the node $d$ is associated with the following factor

$$
\left(-k_{d} \cdot k_{c}\right) \frac{\left\langle c, x_{r}\right\rangle}{\langle c, d\rangle\left\langle d, x_{r}\right\rangle}
$$

Putting the above factors together and summing over $l \in\left(\mathscr{C}_{1} \backslash\left\{x_{r}\right\}\right) \cup \mathscr{C}_{2}$ we get

$$
\begin{aligned}
T_{1}= & \sum_{l \in\left(\mathscr{C}_{1} \backslash\left\{x_{r}\right\}\right) \cup \mathscr{C}_{2}}\left[\left(\epsilon_{h_{j}} \cdot k_{l}\right) \frac{\left\langle l, x_{r}\right\rangle}{\left\langle l, h_{j}\right\rangle\left\langle h_{j}, x_{r}\right\rangle}\right] \\
& \times\left[\prod_{e(x, y) \in \mathcal{E}(\mathscr{C})}\left(\epsilon_{x} \cdot k_{y}\right) \frac{\left\langle y, x_{r}\right\rangle}{\langle y, x\rangle\left\langle x, x_{r}\right\rangle}\right]\left(-k_{d} \cdot k_{c}\right) \frac{\left\langle c, x_{r}\right\rangle}{\langle c, d\rangle\left\langle d, x_{r}\right\rangle} \\
= & \sum_{l \in \mathscr{C}_{1} \cup \mathscr{C}_{2}} \frac{1}{2} \frac{[\xi, l]\left\langle l, x_{r}\right\rangle}{\left[\xi, h_{j}\right]\left\langle h_{j}, x_{r}\right\rangle}\left[\prod_{e(x, y) \in \mathcal{E}(\mathscr{C})}\left(\epsilon_{x} \cdot k_{y}\right) \frac{\left\langle y, x_{r}\right\rangle}{\langle y, x\rangle\left\langle x, x_{r}\right\rangle}\right] \frac{[c, d]\left\langle c, x_{r}\right\rangle}{\left\langle d, x_{r}\right\rangle} \\
= & -\sum_{l \in \mathscr{C}} \frac{1}{2} \frac{[\xi, l][c, d]}{\left[\xi, h_{j}\right]}\left[\frac{\left\langle l, x_{r}\right\rangle\left\langle c, x_{r}\right\rangle}{\left\langle h_{j}, x_{r}\right\rangle\left\langle d, x_{r}\right\rangle}\right]\left[\prod_{e(x, y) \in \mathcal{E}(\mathscr{C})}\left(\epsilon_{x} \cdot k_{y}\right) \frac{\left\langle y, x_{r}\right\rangle}{\langle y, x\rangle\left\langle x, x_{r}\right\rangle}\right],
\end{aligned}
$$

in which, $e(x, y) \in \mathcal{E}(\mathscr{C})$ denoted the set of (type-2) lines in $\mathscr{C}$ and $e(x, y)$ denoted the type-2 line starting at $x$ and ending at $y$, the term with $l=x_{r}$ was included in the second line because of $\left\langle x_{r}, x_{r}\right\rangle=0$, momentum conservation was applied on the third line.

- (b). In figure 14 (b), when nodes in the $\mathscr{C}$ part and the node $d$ are extracted out from the Parke-Taylor factors, we get the following factor

$$
\begin{aligned}
T_{2} & =\left[\sum_{l \in \mathscr{C}}\left(-k_{d} \cdot k_{l}\right) \frac{\left\langle l, x_{r}\right\rangle}{\langle l, d\rangle\left\langle d, x_{r}\right\rangle}\right]\left[\left(\epsilon_{h_{j}} \cdot k_{c}\right) \frac{\left\langle c, x_{r}\right\rangle}{\left\langle c, h_{j}\right\rangle\left\langle h_{j}, x_{r}\right\rangle}\right]\left[\prod_{e(x, y) \in \mathcal{E}(\mathscr{C})}\left(\epsilon_{x} \cdot k_{y}\right) \frac{\left\langle y, x_{r}\right\rangle}{\langle y, x\rangle\left\langle x, x_{r}\right\rangle}\right] \\
& =\sum_{l \in \mathscr{C}} \frac{1}{2} \frac{[l, d][\xi, c]}{\left[\xi, h_{j}\right]}\left[\frac{\left\langle l, x_{r}\right\rangle\left\langle c, x_{r}\right\rangle}{\left\langle d, x_{r}\right\rangle\left\langle h_{j}, x_{r}\right\rangle}\right]\left[\prod_{e(x, y) \in \mathcal{E}(\mathscr{C})}\left(\epsilon_{x} \cdot k_{y}\right) \frac{\left\langle y, x_{r}\right\rangle}{\langle y, x\rangle\left\langle x, x_{r}\right\rangle}\right]
\end{aligned}
$$

where all possible $l \in \mathscr{C}$ were summed over.

- (c). To evaluate figure 14 (c), we suppose the node $l \in \mathscr{C}$ is connected to $c$ via the type-3 line. Assuming that nodes on the path from $l$ to $h_{j}$ are $u_{1}, u_{2}, \ldots, u_{k}$, respectively, all the type- 2 lines between adjacent nodes on this path point away from the trace 1 . When extracting the nodes $u_{1}, \ldots, u_{k}, h_{j}$, from the Parke-Taylor factors 
according to eq. (B.9), we get a factor

$$
\begin{gathered}
\left(-\epsilon_{u_{k}} \cdot k_{h_{j}}\right)\left(-\epsilon_{u_{k-1}} \cdot k_{u_{k}}\right) \ldots\left(-\epsilon_{u_{1}} \cdot k_{u_{2}}\right)\left(-\epsilon_{l} \cdot k_{u_{1}}\right) \frac{\left\langle l, x_{r}\right\rangle}{\left\langle l, u_{1}, \ldots, u_{k}, h_{j}, x_{r}\right\rangle} \\
=\left(\epsilon_{u_{k}} \cdot k_{h_{j}}\right)\left(\epsilon_{u_{k-1}} \cdot k_{u_{k}}\right) \ldots\left(\epsilon_{u_{1}} \cdot k_{u_{2}}\right)\left(\epsilon_{l} \cdot k_{u_{1}}\right) \frac{\left\langle h_{j}, x_{r}\right\rangle}{\left\langle h_{j}, u_{k}, \ldots, u_{1}, l, x_{r}\right\rangle}\left(\frac{\left\langle l, x_{r}\right\rangle}{\left\langle h_{j}, x_{r}\right\rangle}\right)^{2} \\
=\left[\left(\epsilon_{u_{k}} \cdot k_{h_{j}}\right) \frac{\left\langle h_{j}, x_{r}\right\rangle}{\left\langle h_{j}, u_{k}\right\rangle\left\langle u_{k}, x_{r}\right\rangle}\left(\epsilon_{u_{k-1}} \cdot k_{u_{k}}\right) \frac{\left\langle u_{k}, x_{r}\right\rangle}{\left\langle u_{k}, u_{k-1}\right\rangle\left\langle u_{k-1}, x_{r}\right\rangle}\right. \\
\left.\quad \ldots\left(\epsilon_{u_{1}} \cdot k_{u_{2}}\right) \frac{\left\langle u_{2}, x_{r}\right\rangle}{\left\langle u_{2}, u_{1}\right\rangle\left\langle u_{1}, x_{r}\right\rangle}\left(\epsilon_{l} \cdot k_{u_{1}}\right) \frac{\left\langle u_{1}, x_{r}\right\rangle}{\left\langle u_{1}, l\right\rangle\left\langle l, x_{r}\right\rangle}\right]\left(\frac{\left\langle l, x_{r}\right\rangle}{\left\langle h_{j}, x_{r}\right\rangle}\right)^{2}
\end{gathered}
$$

where the antisymmetry of spinor products has been applied. The expression in the square brackets on the last line would have the form as if arrows on this path pointed towards the trace 1 . Arrows of lines in $\mathscr{C}$ which do not on the path from $l$ to $h_{j}$ already point towards the trace $\mathbf{1}$. Hence full contribution of lines in the $\mathscr{C}$ part reads

$$
\left[\prod_{e(x, y) \in \mathcal{E}(\mathscr{C})}\left(\epsilon_{x} \cdot k_{y}\right) \frac{\left\langle y, x_{r}\right\rangle}{\langle y, x\rangle\left\langle x, x_{r}\right\rangle}\right]\left(\frac{\left\langle l, x_{r}\right\rangle}{\left\langle h_{j}, x_{r}\right\rangle}\right)^{2} .
$$

The node $d$ and the node $l$ respectively provide the following factors when they are extracted out

$$
\left(-\epsilon_{h_{j}} \cdot k_{d}\right) \frac{\left\langle h_{j}, x_{r}\right\rangle}{\left\langle h_{j}, d\right\rangle\left\langle d, x_{r}\right\rangle}, \quad\left(-k_{c} \cdot k_{l}\right) \frac{\left\langle c, x_{r}\right\rangle}{\langle c, l\rangle\left\langle l, x_{r}\right\rangle} .
$$

Therefore, the contributions of lines in $\mathscr{C}$ together with the red parts in figure 14 (c) are collected as

$$
\left(-\epsilon_{h_{j}} \cdot k_{d}\right) \frac{\left\langle h_{j}, x_{r}\right\rangle}{\left\langle h_{j}, d\right\rangle\left\langle d, x_{r}\right\rangle}\left(-k_{c} \cdot k_{l}\right) \frac{\left\langle c, x_{r}\right\rangle}{\langle c, l\rangle\left\langle l, x_{r}\right\rangle}\left[\prod_{e(x, y) \in \mathcal{E}(\mathscr{C})}\left(\epsilon_{x} \cdot k_{y}\right) \frac{\left\langle y, x_{r}\right\rangle}{\langle y, x\rangle\left\langle x, x_{r}\right\rangle}\right]\left(\frac{\left\langle l, x_{r}\right\rangle}{\left\langle h_{j}, x_{r}\right\rangle}\right)^{2} .
$$

When spinor expressions are applied and $l \in \mathscr{C}$ is summed over, the above expression turns into

$$
T_{3}=\sum_{l \in \mathscr{C}} \frac{1}{2} \frac{[c, l][\xi, d]}{\left[\xi, h_{j}\right]}\left[\frac{\left\langle l, x_{r}\right\rangle\left\langle c, x_{r}\right\rangle}{\left\langle d, x_{r}\right\rangle\left\langle h_{j}, x_{r}\right\rangle}\right]\left[\prod_{e(x, y) \in \mathcal{E}(\mathscr{C})}\left(\epsilon_{x} \cdot k_{y}\right) \frac{\left\langle y, x_{r}\right\rangle}{\langle y, x\rangle\left\langle x, x_{r}\right\rangle}\right] .
$$

The sum of $T_{1}, T_{2}$ and $T_{3}$ for any $l$ precisely cancel out due to Schouten identity (B.5).

Property-4. Graphs with the structures figure 15 (a), (b) and (c) cancel with each other. Before proving this property, let us first explain the meaning of dashed lines in the graphs: (1) the dashed arrow lines inherit the definition of the type-4 line (see figure $1(\mathrm{~d})$ ) but the two ends of these lines can be gravitons and/or gluons, (2). the dashed line with no arrow in figure 15 (colored red) also reflects the relative position between its two end nodes, but it does not bring any sign. The cancellation between graphs figure 15 (a), (b) and (c) only relies on the relative positions between nodes and possible signs caused by the arrows (just as the property-3). Let us now evaluate the different parts in the three graphs in turn. 


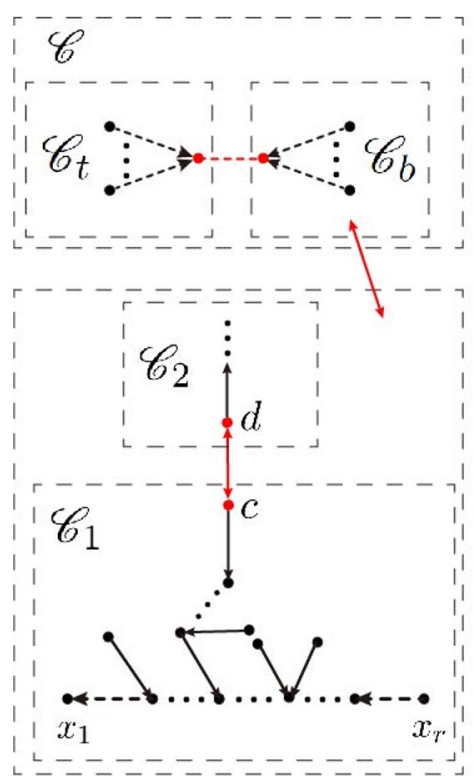

(a)

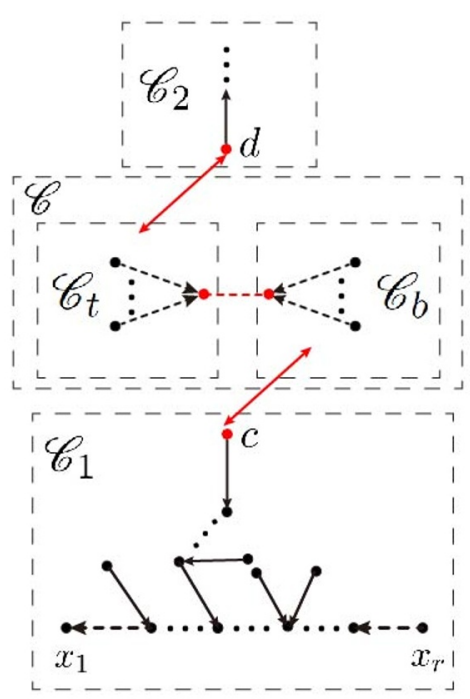

(b)

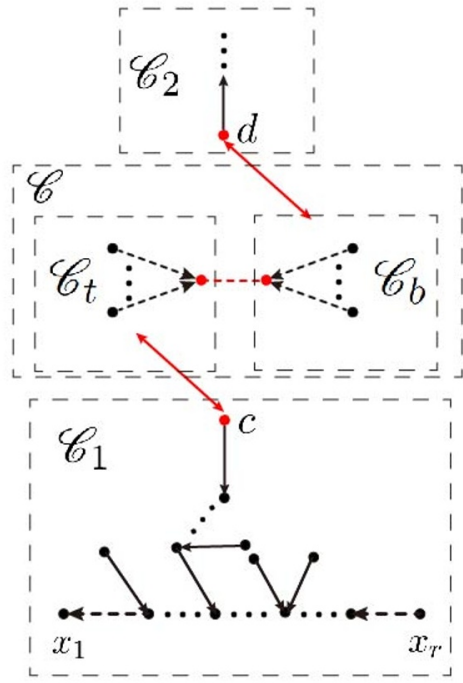

(c)

Figure 15. Contributions of graphs with the above typical structures cancel out. Here, the substructure $\mathscr{C}$ is divided into two parts $\mathscr{C}_{t}$ and $\mathscr{C}_{b}$ by a dashed line with no arrow.

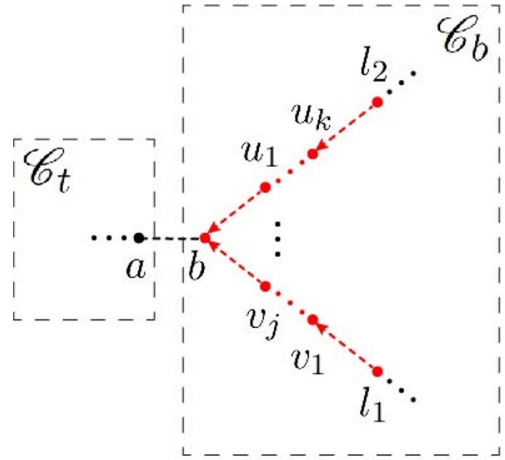

(a)

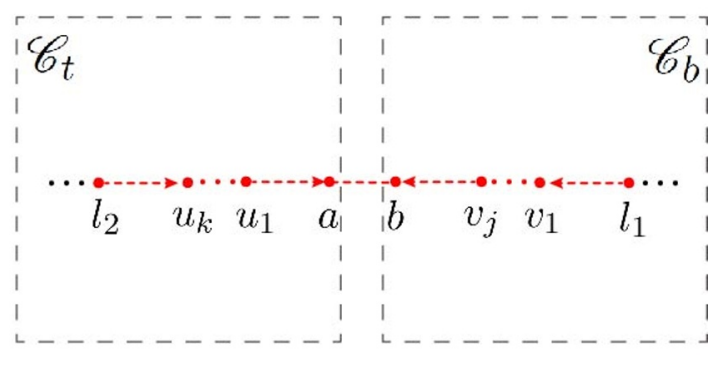

(b)

Figure 16. (a): the path between $l_{1}$ and $l_{2}$ in eq. (5.13), (b): the path between $l_{2}$ and $l_{1}$ in figure 15 (c).

- (a) Suppose that the two end nodes of the type-3 line between $\mathscr{C}_{b}$ and $\mathscr{C}_{1} \cup \mathscr{C}_{2}$ in figure 15 (a) are respectively $l_{1} \in \mathscr{C}_{b}$ and $l_{2} \in\left(\mathscr{C}_{1} \backslash\left\{x_{r}\right\}\right) \cup \mathscr{C}_{2}$. Then all lines in $\mathscr{C}$, the type-3 line between $l_{1}, l_{2}$ and the type-3 line between $c, d$ together produce the following factor

$$
\sum_{l_{2} \in\left(\mathscr{C}_{1} \backslash\left\{x_{r}\right\}\right) \cup \mathscr{C}_{2}} \sum_{l_{1} \in \mathscr{C}_{b}} T\left[\mathscr{C}\left(l_{1}\right)\right]\left[\left(-k_{l_{1}} \cdot k_{l_{2}}\right) \frac{\left\langle l_{2}, x_{r}\right\rangle}{\left\langle l_{2}, l_{1}\right\rangle\left\langle l_{1}, x_{r}\right\rangle}\right]\left[\left(-k_{d} \cdot k_{c}\right) \frac{\left\langle c, x_{r}\right\rangle}{\langle c, d\rangle\left\langle d, x_{r}\right\rangle}\right],
$$

where $T\left[\mathscr{C}\left(l_{1}\right)\right]$ denotes the full contributions of lines in the region $\mathscr{C}$ when $l_{1}$ is the node nearest to the trace 1 . Momentum conservation allows one to convert the 
summation over $l_{2} \in\left(\mathscr{C}_{1} \backslash\left\{x_{r}\right\}\right) \cup \mathscr{C}_{2}$ into a summation over $l_{2} \in \mathscr{C}_{t} \cup \mathscr{C}_{b}$ with an extra sign $(-1)$, which further splits into the summations over $l_{2} \in \mathscr{C}_{b}$ and $l_{2} \in \mathscr{C}_{t}$. The sum of terms with $l_{2} \in \mathscr{C}_{b}$ is explicitly written as

$$
-\sum_{l_{1}, l_{2} \in \mathscr{C}_{b}} T\left[\mathscr{C}\left(l_{1}\right)\right]\left[\left(-k_{l_{1}} \cdot k_{l_{2}}\right) \frac{\left\langle l_{2}, x_{r}\right\rangle}{\left\langle l_{2}, l_{1}\right\rangle\left\langle l_{1}, x_{r}\right\rangle}\right]\left[\left(-k_{d} \cdot k_{c}\right) \frac{\left\langle c, x_{r}\right\rangle}{\langle c, d\rangle\left\langle d, x_{r}\right\rangle}\right] .
$$

For a given $l_{1}, l_{2} \in \mathscr{C}_{b}$ in the above expression, one can always find out another term in the summation by exchanging the roles of $l_{1}$ and $l_{2}$. The only difference between $T\left[\mathscr{C}\left(l_{1}\right)\right]$ and $T\left[\mathscr{C}\left(l_{2}\right)\right]$ is the factor corresponding to the path between $l_{1}$ and $l_{2}$ (see figure 16 (a)). Supposing the nodes between $l_{1}$ and $l_{2}$ are in turn $l_{1}, v_{1}, \ldots, v_{j}, b, u_{1}, \ldots, u_{k}, l_{2}$, the lines between $l_{1}$ and $l_{2}$ contribute the following factor to $T\left[\mathscr{C}\left(l_{1}\right)\right]$ :

$$
(-1)^{j+1} \frac{\left\langle l_{1}, x_{r}\right\rangle}{\left\langle l_{1}, v_{1}, \ldots, v_{j}, b, u_{1}, \ldots, u_{k}, l_{2}, x_{r}\right\rangle}=\frac{\left\langle l_{1}, x_{r}\right\rangle}{\left\langle b, v_{j}, \ldots, v_{1}, l_{1}\right\rangle\left\langle b, u_{1}, \ldots, u_{k}, l_{2}, x_{r}\right\rangle},
$$

when eq. (B.9) is been applied. Together with the second factor in eq. (5.13), the above factor provides

$$
\begin{aligned}
& \frac{\left\langle l_{1}, x_{r}\right\rangle}{\left\langle b, v_{j}, \ldots, v_{1}, l_{1}\right\rangle\left\langle b, u_{1}, \ldots, u_{k}, l_{2}, x_{r}\right\rangle}\left(-k_{l_{1}} \cdot k_{l_{2}}\right) \frac{\left\langle l_{2}, x_{r}\right\rangle}{\left\langle l_{2}, l_{1}\right\rangle\left\langle l_{1}, x_{r}\right\rangle} \\
& =\frac{1}{2} \frac{\left[l_{2}, l_{1}\right]}{\left\langle b, v_{j}, \ldots, v_{1}, l_{1}\right\rangle\left\langle b, u_{1}, \ldots, u_{k}, l_{2}\right\rangle} .
\end{aligned}
$$

While exchanging the roles of $l_{1}$ and $l_{2}$, we get the same expression with an opposite sign. This antisymmetry indicates that eq. (5.13) vanishes when all $l_{1}, l_{2} \in \mathscr{C}_{b}$ are summed over. The expression (5.12) is then reduced into

$$
\begin{aligned}
T_{1} & =-\sum_{l_{2} \in \mathscr{C}_{b}} \sum_{l_{1} \in \mathscr{C}_{b}} T\left[\mathscr{C}\left(l_{1}\right)\right]\left[\left(-k_{l_{1}} \cdot k_{l_{2}}\right) \frac{\left\langle l_{2}, x_{r}\right\rangle}{\left\langle l_{2}, l_{1}\right\rangle\left\langle l_{1}, x_{r}\right\rangle}\right]\left[\left(-k_{d} \cdot k_{c}\right) \frac{\left\langle c, x_{r}\right\rangle}{\langle c, d\rangle\left\langle d, x_{r}\right\rangle}\right] \\
& =\sum_{l_{2} \in \mathscr{C}_{t}} \sum_{l_{1} \in \mathscr{C}_{b}} \frac{1}{2}\left[l_{1}, l_{2}\right][c, d] \frac{\left\langle l_{2}, x_{r}\right\rangle\left\langle c, x_{r}\right\rangle}{\left\langle l_{1}, x_{r}\right\rangle\left\langle d, x_{r}\right\rangle} T\left[\mathscr{C}\left(l_{1}\right)\right] .
\end{aligned}
$$

- (b) In figure 15 (b), the two type-3 lines and the $\mathscr{C}$ part together contribute a factor

$$
\begin{aligned}
T_{2} & =\sum_{l_{2} \in \mathscr{C}_{t}} \sum_{l_{1} \in \mathscr{C}_{b}}\left(-k_{d} \cdot k_{l_{2}}\right) \frac{\left\langle l_{2}, x_{r}\right\rangle}{\left\langle l_{2}, d\right\rangle\left\langle d, x_{r}\right\rangle}\left(-k_{c} \cdot k_{l_{1}}\right) \frac{\left\langle c, x_{r}\right\rangle}{\left\langle c, l_{1}\right\rangle\left\langle l_{1}, x_{r}\right\rangle} T\left[\mathscr{C}\left(l_{1}\right)\right] \\
& =\sum_{l_{2} \in \mathscr{C}_{t}} \sum_{l_{1} \in \mathscr{C}_{b}} \frac{1}{2}\left[l_{2}, d\right]\left[c, l_{1}\right] \frac{\left\langle l_{2}, x_{r}\right\rangle\left\langle c, x_{r}\right\rangle}{\left\langle l_{1}, x_{r}\right\rangle\left\langle d, x_{r}\right\rangle} T\left[\mathscr{C}\left(l_{1}\right)\right] .
\end{aligned}
$$

- (c) In figure 15 (c), the two type-3 lines and the $\mathscr{C}$ part contribute the following factor

$$
\begin{aligned}
T_{3} & =\sum_{l_{2} \in \mathscr{C}_{t}} \sum_{l_{1} \in \mathscr{C}_{b}}\left(-k_{d} \cdot k_{l_{1}}\right) \frac{\left\langle l_{1}, x_{r}\right\rangle}{\left\langle l_{1}, d\right\rangle\left\langle d, x_{r}\right\rangle}\left(-k_{c} \cdot k_{l_{2}}\right) \frac{\left\langle c, x_{r}\right\rangle}{\left\langle c, l_{2}\right\rangle\left\langle l_{2}, x_{r}\right\rangle} T\left[\mathscr{C}\left(l_{2}\right)\right] \\
& =\sum_{l_{2} \in \mathscr{C}_{t}} \sum_{l_{1} \in \mathscr{C}_{b}} \frac{1}{2}\left[l_{1}, d\right]\left[c, l_{2}\right] \frac{\left\langle l_{1}, x_{r}\right\rangle\left\langle c, x_{r}\right\rangle}{\left\langle l_{2}, x_{r}\right\rangle\left\langle d, x_{r}\right\rangle} T\left[\mathscr{C}\left(l_{2}\right)\right] .
\end{aligned}
$$


To relate the $T\left[\mathscr{C}\left(l_{2}\right)\right]$, which is the expression of $\mathscr{C}$ part while $l_{2}$ is the node nearest to the trace 1 , to $T\left[\mathscr{C}\left(l_{1}\right)\right]$, we note that the only difference between $T\left[\mathscr{C}\left(l_{1}\right)\right]$ and $T\left[\mathscr{C}\left(l_{2}\right)\right]$ is the path between the nodes $l_{1} \in \mathscr{C}_{b}$ and $l_{2} \in \mathscr{C}_{t}$ (see figure $16(\mathrm{~b})$ ). In $T\left[\mathscr{C}\left(l_{2}\right)\right]$, this path has the form

$$
(-1)^{k+1} \frac{\left\langle l_{2}, x_{r}\right\rangle}{\left\langle l_{2}, u_{k}, \ldots, u_{1}, a, b, v_{1}, \ldots, v_{j}, l_{1}, x_{r}\right\rangle}=\frac{\left\langle l_{2}, x_{r}\right\rangle}{\left\langle a, u_{1}, \ldots, u_{k}, l_{2}\right\rangle\langle a, b\rangle\left\langle b, v_{1}, \ldots, v_{j}, l_{1}, x_{r}\right\rangle},
$$

where nodes on this path were supposed to be $l_{2}, u_{k}, \ldots, u_{1}, a, b, v_{1}, \ldots, v_{j}, l_{1}$ in turn. Similarly, in $T\left[\mathscr{C}\left(l_{1}\right)\right]$, the path from $l_{1}$ to $l_{2}$ contributes a factor

$$
\frac{\left\langle l_{1}, x_{r}\right\rangle}{\left\langle b, v_{1}, \ldots, v_{j}, l_{1}\right\rangle\langle b, a\rangle\left\langle a, u_{1}, \ldots, u_{k}, l_{2}, x_{r}\right\rangle} .
$$

Therefore, we arrive $T\left[\mathscr{C}\left(l_{2}\right)\right]=\left(-\frac{\left\langle l_{2}, x_{r}\right\rangle^{2}}{\left\langle l_{1}, x_{r}\right\rangle^{2}}\right) T\left[\mathscr{C}\left(l_{1}\right)\right]$ and $T_{3}$ is immediately rewritten as

$$
T_{3}=\sum_{l_{2} \in \mathscr{C}_{t}} \sum_{l_{1} \in \mathscr{C}_{b}} \frac{1}{2}\left[d, l_{1}\right]\left[c, l_{2}\right] \frac{\left\langle l_{2}, x_{r}\right\rangle\left\langle c, x_{r}\right\rangle}{\left\langle l_{1}, x_{r}\right\rangle\left\langle d, x_{r}\right\rangle} T\left[\mathscr{C}\left(l_{1}\right)\right] .
$$

When summing $T_{1}, T_{2}$ and $T_{3}$ together, we get

$$
T_{1}+T_{2}+T_{3}=\sum_{l_{2} \in \mathscr{C}_{t}} \sum_{l_{1} \in \mathscr{C}_{b}} \frac{1}{2}\left(\left[l_{1}, l_{2}\right][c, d]+\left[l_{2}, d\right]\left[c, l_{1}\right]+\left[d, l_{1}\right]\left[c, l_{2}\right]\right) \frac{\left\langle l_{2}, x_{r}\right\rangle\left\langle c, x_{r}\right\rangle}{\left\langle l_{1}, x_{r}\right\rangle\left\langle d, x_{r}\right\rangle} T\left[\mathscr{C}\left(l_{1}\right)\right],
$$

which precisely vanishes due to the Schouten identity (B.5). Thus we conclude that the graphs figure 15 (a), (b) and (c) cancel with each other.

\subsection{The vanishing amplitudes}

Having the above properties, we are able to prove the vanishing of all the remaining amplitudes with two negative-helicity particles.

Single-trace amplitudes with the $\left(\boldsymbol{h}_{\boldsymbol{i}}^{-}, \boldsymbol{h}_{j}^{-}\right)$configuration. When evaluating a single-trace amplitude with the $\left(h_{i}^{-}, h_{j}^{-}\right)$configuration, we have to investigate not only (i). graphs in the $M H V$ sector which do not involve the type-3 line but also (ii). graphs with one type-1 line (i.e. graphs in the NMHV sector). This comes from our choice of gauge: those graphs involving the type- 1 line $\epsilon_{h_{j}}^{-} \cdot \epsilon^{+}$do not vanish. We now show cancellations occur in each class of graphs.

(i). The cancellation of graphs in the $M H V$ sector. In a graph with no type- 1 component, the graviton $h_{i}$ cannot be the end node of any type-2 line that starts at a positive-helicity graviton because $\epsilon^{+} \cdot k_{h_{i}}=0$ under our choice of gauge. Hence $h_{i}$ can only be (1). a leaf or (2). an end node of a type-2 line that starts at the other negative-helicity graviton $h_{j}$. According to property-1, graphs in the former case are further reduced to graphs with $h_{i}$ removed which have the pattern figure 13 (a). Graphs in the latter case have the pattern figure 13 (b). Therefore, all graphs cancel in pairs due to property-2. 


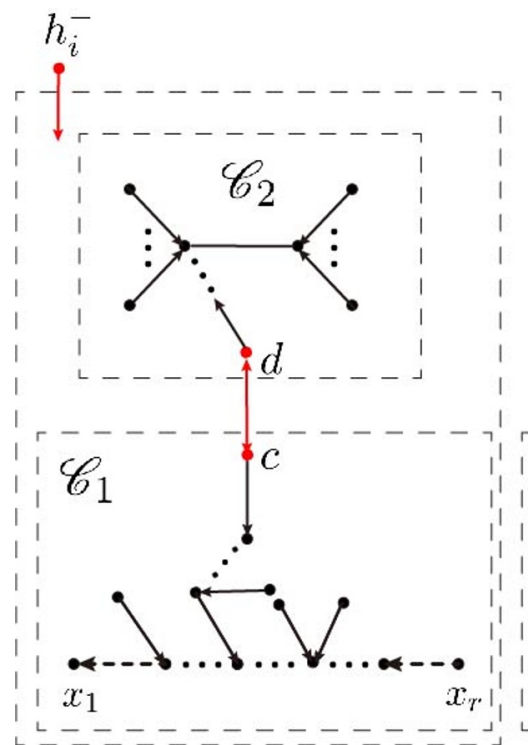

(a)

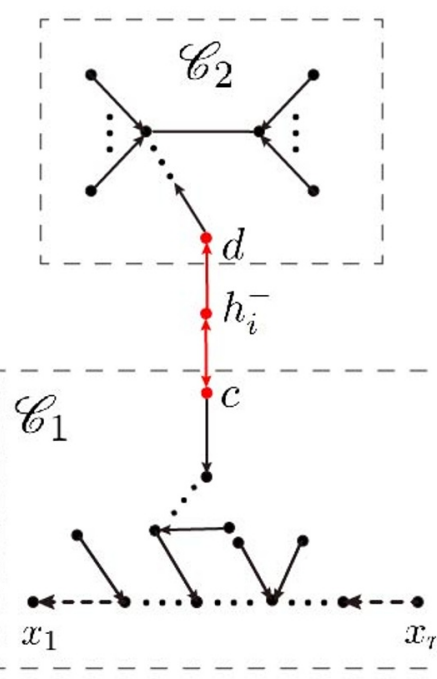

(b)

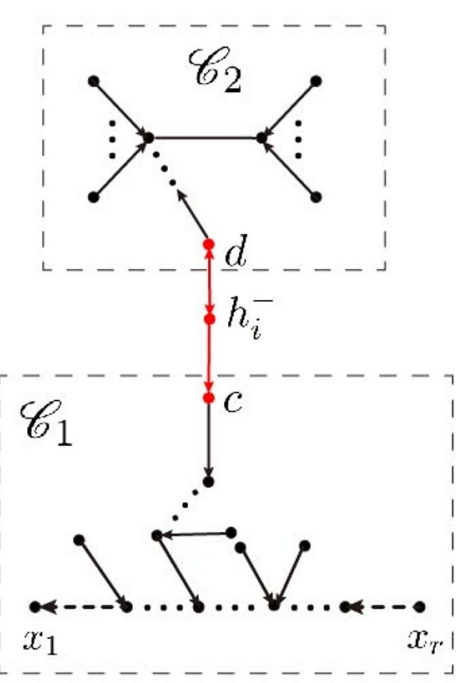

(c)

Figure 17. Typical graphs involving a type-1 line for a single-trace amplitude with the $\left(h_{i}^{-}, h_{j}^{-}\right)$ configuration.

(ii). The cancellation of graphs with one type-1 line $\left(\epsilon_{h_{j}}^{-} \cdot \epsilon^{+}\right)$. In this case, we have two components that are separated by a type-3 $(k \cdot k)$ line (see appendix A): the component invloving the type-1 line $\left(\epsilon_{h_{j}}^{-} \cdot \epsilon^{+}\right)$and the component involving the trace. According to our choice of gauge, the graviton $h_{i}$ can be either a leaf (see figure 17 (a)) or an end node of the type-3 line between the two components (see figure 17 (b) and (c)). The node $h_{i}$ cannot be the end node of a type-2 line that starts at $h_{j}$ because $\epsilon_{h_{j}}$ is already occupied by the type-1 line $\left(\epsilon_{h_{j}}^{-} \cdot \epsilon^{+}\right)$in this case. Graphs figure $17(\mathrm{a}),(\mathrm{b})$ and (c) agree with the pattern of graphs figure 14 (a), (b) and (c) (when $\mathscr{C}$ in figure 14 includes only the graviton $h_{j}$ and $\mathscr{C}_{2}$ denotes the component involving the type-1 line), thus they cancel out due to property-3.

Double-trace amplitudes with the $\left(\boldsymbol{h}_{\boldsymbol{i}}^{-}, \boldsymbol{g}_{j}^{-}\right)$configuration. Now we turn to doubletrace amplitudes with the $\left(h_{i}^{-}, g_{j}^{-}\right)$configuration, all graphs for this case are graphs in the MHV sector, as shown by figure 2 (b). According to our choice of gauge, $h_{i}$ cannot be the end node of a type-2 line which starts at a positive-helicity graviton. Hence $h_{i}$ can only be a leaf or an end node of the type-3 line. The typical graphs can be obtained via replacing the $\mathscr{C}_{2}$ in figure 17 (a), (b) and (c) by the component (which is defined by figure 18 (b) in appendix A) involving the trace 2 . Since the patterns of graphs figure 17 (a), (b) and (c) are preserved, they must cancel out due to property-3. Thus we conclude that an amplitude with the $\left(h_{i}^{-}, g_{j}^{-}\right)$configuration has to vanish.

Double-trace amplitudes with the $\left(\boldsymbol{h}_{\boldsymbol{i}}^{-}, \boldsymbol{h}_{\boldsymbol{j}}^{-}\right)$configuration. When we evaluate a double-trace amplitude with the $\left(h_{i}^{-}, h_{j}^{-}\right)$configuration, a graph may or may not involve a type- 1 line of the form $\left(\epsilon_{h_{j}}^{-} \cdot \epsilon^{+}\right)$. This is analogue to the single-trace case with the $\left(h_{i}^{-}, h_{j}^{-}\right)$ configuration. We state that graphs in each case cancel out. 
(i). The cancellation of graphs with no type-1 line (i.e. graphs in the MHV sector, as shown by figure 2 (b)). All graphs in this case can be classified according to positions of the negative-helicity graviton $h_{j}$ (and the tree structure attached to it). Specifically, $h_{j}$ can be an outer node or an inner node which lives on the bridge between the two traces. The former case is described by figure 14 (a) while the latter case has the pattern figure 14 (b) or (c). Here, the $\mathscr{C}_{2}$ component is the component which involves the trace 2 (see figure 18 (b)). According to property-3, all these graphs cancel with each other.

(ii). The cancellation of graphs involving a type-1 line of the form $\epsilon_{h_{j}}^{-} \cdot \epsilon^{+}$(i.e. graphs in the NMHV sector). According to the refined graphic rule in appendix A, each graph in this case involves three components with the patterns figure 18 (a), (b) and (c) which respectively contain the type- 1 line $\epsilon_{h_{j}}^{-} \cdot \epsilon^{+}$, the trace 2 and the trace 1 . These graphs can be classified according to whether the type- 1 line is on the bridges between the two traces or not. When the common kinematic factors $\epsilon \cdot k$ and $\epsilon_{h_{j}}^{-} \cdot \epsilon^{+}$in the component containing the type- 1 line are extracted out, graphs in this case precisely match with the patterns figure 15 (a), (b) and (c) (with the correct arrows), while $\mathscr{C}_{1}$ and $\mathscr{C}_{2}$ respectively denote the components containing traces 1 and 2 . Then we conclude that these graphs cancel with each other due to property- 4 .

Amplitudes with more than two traces. For amplitudes with more than two traces and arbitrary two negative-helicity particles, there exist at least three components which are connected together by type-3 lines. In order to avoid a tedious discussion, we just sketch the main pattern of the cancellation of amplitudes in this case. As stated in [10], an amplitude with more than two traces can be obtained by (i). constructing so-called the upper and lower blocks, the latter involves the trace 1, (ii). attaching a substructure $\mathscr{C}$, which is divided by the kernel of either a type-IA or a type-IB component (see figure 18 (a), (b)), to either the upper or the lower block constructed in the previous step, (iii). connecting the two disconnected subgraphs obtained in the previous step via a type-3 line. In a graph constructed by (i)-(iii), the kernel of the substructure $\mathscr{C}$ may be or may not be on the bridge between the upper and lower blocks. Correspondingly, when the kinematic factor of $\mathscr{C}$ is extracted out, such a graph has the pattern figure 15 (b), (c) or (a), accompanied by the correct sign. Therefore, all graphs with the same $\mathscr{C}$ and the same configuration of upper and lower blocks must cancel out due to property-4.

\section{Conclusions and further discussions}

In this work, we evaluated all tree level EYM amplitudes with two negative-helicity particles and an arbitrary number of traces in four dimensions, by the use of refined graphic rule. According to the number of $\epsilon \cdot \epsilon$ lines in the graphs, all graphs were classified into $\mathrm{N}^{k} \mathrm{MHV}$ sectors. We pointed that the nonvanishing amplitudes with $k+2$ negative-helicity particles could at most get contributions from graphs in the $\mathrm{N}^{k^{\prime}\left(k^{\prime} \leq k\right)} \mathrm{MHV}$ sectors, under a proper choice of gauge. For single-trace amplitudes with the $\left(g_{i}^{-}, g_{j}^{-}\right)$and the $\left(h_{i}^{-}, g_{j}^{-}\right)$ configurations, we established the correspondence between the refined graphs and the spanning forests in four dimensions. A symmetric formula of double-trace amplitudes with the 
$\left(g_{i}^{-}, g_{j}^{-}\right)$configuration was further provided. In this formula, the two gluon traces are on an equal footing while all gravitons are disentangled from the Parke-Taylor factors corresponding to gluon traces. Other amplitudes with two negative-helicity particles were proven to vanish, due to properties of the graphs. In the end of this paper, we provide further discussions on related topics:

(i). When we take the soft limit of a graviton in eq. (3.1) or eq. (4.1), the correct soft factor (which was first proposed in [37]) is obtained. The inverse process of soft limit (ISL), as pointed in [38-41] has been used to reconstruct the full amplitude. Especially, the graphic representation [39] of graviton MHV amplitudes can be reproduced by the same soft factor given in [37]. This inspires that the formulas (3.1) and (4.1) for MHV amplitudes in EYM could also be constructed by the ISL. We have verified this point by reconstructing single-trace amplitudes with one and two gravitons as well as the double-trace amplitude with one graviton. We leave the general discussion in future work.

(ii). How to extend the discussions to amplitudes with more than two negative-helicity particles? Since the refined graphic rule is independent of dimensions, the discussion of this paper can be extended to configurations with more negative-helicity particles. Nevertheless, the general formula of $\mathrm{N}^{k(k>2)} \mathrm{MHV}$ amplitudes in YM depends on the position of negative-helicity particles [42]. This may require more hidden properties of the refined graphs in four dimensions.

(iii). It is worth studying amplitudes with gravitons coupling to matters in other theories. As pointed in $[18,19]$, the recursive expansion of EYM amplitudes could be generalized to other theories with gravitons coupling to matters. Hence we expect that the calculations in the current paper, by the use of refined graphic rule (which is based on the recursive expansion of EYM amplitudes), can also be extended to these theories.

\section{Acknowledgments}

We would like to thank Linghui Hou, Jingpei Liu and Konglong Wu for helpful discussions. We also thank the referee for valuable suggestions. This work is supported by NSFC under Grant No. 11875206, Jiangsu Ministry of Science and Technology under contract BK20170410 as well as the "Fundamental Research Funds for the Central Universities".

\section{A Sectors in the graphic expansion of EYM amplitudes}

In this part, we present the refined graphic rule for the $\mathrm{N}^{k} \mathrm{MHV}$ sector in the expansion (2.3). This rule follows from a discussion parallel with those in $[8,10]$.

\section{A.1 The construction of a graph $\mathcal{F} \in \mathcal{F}[k, m]$ in the $\mathrm{N}^{k} \mathrm{MHV}$ sector}

To construct a graph $\mathcal{F} \in \mathcal{F}[k, m]$ in the $\mathrm{N}^{k} \mathrm{MHV}$ sector, we introduce the set of all gravitons and the gluon traces $\mathbf{2}, \ldots, \boldsymbol{m}, \boldsymbol{H}=\left\{h_{1}, h_{2}, \ldots, h_{s}, \mathbf{2}, \ldots, \boldsymbol{m}\right\}$. Each element in 


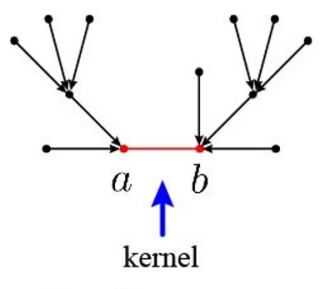

Type-IA component

(a)

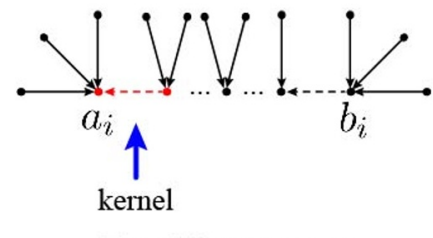

(b)

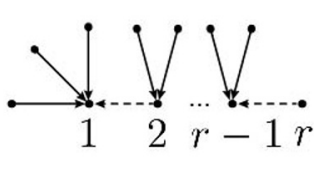

Type-II component

(c)

Figure 18. Three types of components defined in the refined graphic rule.

this set is denoted by $\mathcal{H}_{i}(i=1, \ldots, m+s-1)$. We further define a reference order $\mathrm{R}$ by the ordered set

$$
\mathrm{R}=\left\{\mathcal{H}_{\rho(1)}, \mathcal{H}_{\rho(2)}, \ldots, \mathcal{H}_{\rho(l=m+s-1)}\right\}
$$

where each gluon trace is considered as a single object. The position of an element in the reference order $\mathrm{R}$ is called its weight. When all gravitons and gluons are treated as nodes, a graph $\mathcal{F}[k, m]$ is obtained by connecting lines (see figure 1 ) between these nodes in an appropriate way. This can be achieved by the following two steps. (i). Construct a skeleton that does not involve any type-3 line and may contain more than one mutually disjoint maximally connected subgraphs (which are defined as components). (ii). Connect these components of a skeleton via type-3 lines such that the skeleton becomes a fully connected tree graph $\mathcal{F}[k, m]$. Now we look into details of these two steps respectively.

Step-1. Constructing a skeleton. To obtain a skeleton, we first connect type-4 lines between adjacent nodes in each trace. For the trace $\mathbf{1}$, the nodes are arranged in the relative order $1,2, \ldots, r$ and all type- 4 lines in 1 point towards the direction of the node 1 . For the trace $\boldsymbol{i}(\boldsymbol{i} \neq \mathbf{1})$, the gluons therein are arranged in a relative order

$$
a_{i}, \beta_{1}, \ldots, \beta_{|\boldsymbol{i}|-2}, b_{i}, \quad\left(\left\{\beta_{1}, \ldots, \beta_{\left|\boldsymbol{t}_{i}\right|-2}\right\} \equiv \boldsymbol{\beta} \in \mathrm{KK}\left[\boldsymbol{i}, a_{i}, b_{i}\right]\right) .
$$

where $\mathrm{KK}\left[\boldsymbol{i}, a_{i}, b_{i}\right]=\boldsymbol{X} ш \boldsymbol{Y}^{T}$ when the trace $\boldsymbol{i}$ is supposed to have the form $a_{i}, \boldsymbol{X}, b_{i}, \boldsymbol{Y}$. All the type- 4 lines in $\boldsymbol{i}$ point towards the gluon $a_{i}$. We then pick out $k$ pairs of gravitons and connect a type- 1 line between each pair. A type- 1 line between two gravitons or the type- 4 line which is attached to the ending gluon $a_{i}$ of a trace is called a kernel. All other gravitons are connected to either gluons (the gluon $r \in \mathbf{1}$ is excluded) in traces or the graviton pairs with a type- 1 line between them, through type- 2 lines. Respectively, the arrows of these type- 2 lines point towards the trace or the graviton pairs. Then a skeleton generally consists of the following two types of components:

- Type-I components. Components which do not involve the trace $\mathbf{1}$. This type of components can be further classified into type-IA and type-IB components. A typeIA component is defined by a component consisting of only gravitons (as shown by figure 18 (a)), while a type-IB component is defined by a component with a gluon trace $\boldsymbol{i}(\boldsymbol{i} \neq \mathbf{1})$ in it (as shown by figure $18(\mathrm{~b}))$. We define the weight of each type-IA or -IB component by the weight of its highest-weight node (the weight of a trace $\boldsymbol{i}$ is always 
carried by its fixed gluon $a_{i}$ in (A.2)). Noting that each type-IA or -IB component is divided into two parts by the kernel, we further define the part containing the highest-weight node as the top side, while the opposite part the bottom side.

- Type-II component. Components involving the trace 1. The typical structure of this component is shown by figure 18 (c). Note that the gluon $r$ cannot be connected by any type-2 line.

Since a graph $\mathcal{F}$ in the $\mathrm{N}^{k} \mathrm{MHV}$ sector for an $m$-trace amplitude has $k$ type- 1 lines, its skeleton must involve $k$ type-IA components, $m-1$ type-IB components and one type-II component.

Step-2. Constructing graphs corresponding to a skeleton. Having a skeleton with $k$ type-IA components, $m-1$ type-IB components and one type-II component, we need to connect these components into a fully connected graph $\mathcal{F}$. This can be achieved as follows:

(i). For a given skeleton, we define the reference order $\mathrm{R}_{\mathscr{C}}$ of all type-I components (including type-IA and type-IB components) by the relative order of the highest-weight nodes therein. We further define the root set $\mathcal{R} \backslash\{r\}$ by the nodes (except the gluon $r \in \mathbf{1})$ in the type-II component.

(ii). Supposing the reference order of components is $\mathrm{R}_{\mathscr{C}}=\left\{\mathscr{C}_{1}, \mathscr{C}_{2}, \ldots, \mathscr{C}_{N}\right\}$, we pick out the highest-weight component $\mathscr{C}_{N}$ and some other components $\mathscr{C}_{i_{1}}, \mathscr{C}_{i_{2}}, \ldots, \mathscr{C}_{i_{j}}$ (which are not necessarily in the relative order in $R_{\mathscr{C}}$ ), then construct a chain that starts from $\mathscr{C}_{N}$, passes through $\mathscr{C}_{i_{j}}, \mathscr{C}_{i_{j-1}}, \ldots, \mathscr{C}_{i_{1}}$ and ends at a node belonging to $\mathcal{R} \backslash\{r\}$ as follows:

$$
\mathbb{C H}=\left[\left(\mathscr{C}_{N}\right)_{t},\left(\mathscr{C}_{N}\right)_{b} \leftrightarrow\left(\mathscr{C}_{i_{j}}\right)_{t(\text { orb })},\left(\mathscr{C}_{i_{j}}\right)_{b(\text { or } t)} \leftrightarrow \cdots \leftrightarrow\left(\mathscr{C}_{i_{1}}\right)_{t(\text { or } b)},\left(\mathscr{C}_{i_{1}}\right)_{b(\text { or } t)} \leftrightarrow \mathcal{R} \backslash\{r\}\right],
$$

where we have used the subscripts $t, b$ to denote the top and bottom sides respectively. The notation $\leftrightarrow$ stands for the type-3 line between two nodes living in the corresponding regions. Redefine the reference order by removing those components which have been used

$$
\mathrm{R}_{\mathscr{C}} \rightarrow \mathrm{R} \mathscr{C} \backslash\left\{\mathscr{C}_{N}, \mathscr{C}_{i_{1}}, \mathscr{C}_{i_{2}}, \ldots, \mathscr{C}_{i_{j}}\right\}
$$

and redefine the root set by

$$
\mathcal{R} \backslash\{r\} \rightarrow(\mathcal{R} \backslash\{r\}) \cup \mathscr{C}_{i_{1}} \cup \cdots \cup \mathscr{C}_{i_{j}} \cup \mathscr{C}_{N}
$$

(iii). Repeating the above step iteratively until the ordered set $\mathrm{R}_{\mathscr{C}}$ becomes empty, we get a fully connected tree graph $\mathcal{F} \in \mathcal{F}[k, m]$ which is rooted at the gluon $1 \in \mathbf{1}$.

After summing over all possible graphs $\mathcal{F}$ including (a). all choices of $a_{i} \neq b_{i}$ for fixed $b_{i}$ for all traces $\boldsymbol{i} \neq 1$, (b). all possible permutations $\boldsymbol{\beta}_{i} \in \mathrm{KK}\left[\boldsymbol{i}, a_{i}, b_{i}\right]$ for a given trace $\boldsymbol{i}$ and (c). all skeletons constructed for given $a_{i}$ and $\beta_{i}$ and (d). all graphs corresponding to a given skeleton, we get the $\mathrm{N}^{k} \mathrm{MHV}$ sector in eq. (2.3). 


\section{A.2 Coefficient, sign and permutations associated to $\mathcal{F}$}

The coefficient $\mathcal{C}^{\mathcal{F}}$ corresponding to a graph $\mathcal{F}$ is straightforwardly given by the product of factors corresponding to all lines in it (see figure 1). The sign $(-)^{\mathcal{F}}$ in eq. (2.3) is defined as follows: (a). Each arrow pointing away from the gluon $1 \in 1$ contributes a minus. (b). For any type-IB component, if the kernel points away from the root (i.e. the gluon $1 \in \mathbf{1})$, an extra minus must be dressed. (c). Each gluon trace $\boldsymbol{i}(\boldsymbol{i} \neq \mathbf{1})$ is accompanied by $(-1)^{\left|\boldsymbol{i}, a_{i}, b_{i}\right|}$, where $\left|\boldsymbol{i}, a_{i}, b_{i}\right|$ denotes the number of elements in $\boldsymbol{Y}$ if the trace has the form $\boldsymbol{i}=\left\{a_{i}, \boldsymbol{X}, b_{i}, \boldsymbol{Y}\right\}$.

Permutations $\left.\boldsymbol{\sigma} \in \mathcal{F}\right|_{1} \backslash\{1, r\}$ in eq. (2.3) are determined as follows: (a) For two adjacent nodes $a$ and $b$, if $a$ is nearer to 1 than $b$, we have $a \prec b$. (b). If two branches are connected to a same node, we shuffle the relative orders of the nodes belonging to these two branches.

\section{A.3 Graphs in the MHV sectors of single- and double-trace amplitudes}

According to the general rule, a graph $\mathcal{F} \in \mathcal{F}[0,1]$ in the MHV sector of a single-trace EYM amplitude has the pattern figure 2 (a) which involves only one component: the type-II component. These graphs at single-trace level are independent of the choice of reference order because only graphs with more than one components rely on the choice of reference order.

When we choose the reference order as

$$
\mathrm{R}=\left\{h_{1}, h_{2}, \ldots, 2\right\},
$$

i.e., the trace $\mathbf{2}$ is the highest-weight element, a typical graph $\mathcal{F} \in \mathcal{F}[0,2]$ in the MHV sector of a double-trace amplitude is then presented by figure 2 (b). In figure 2 (b), there is a path between the ending node $a$ of the trace 2 and a gluon $l \in 1 \backslash\left\{x_{r}\right\}$. On this path, there exists one type- 3 line and possible type- 2 lines. The arrows of the type- 2 lines above (or below) the type-3 line point towards the node $a \in \mathbf{2}$ (or $l \in \mathbf{1} \backslash\left\{x_{r}\right\}$ ). Other gravitons that do not live on the path from $a$ to $l$, can be connected to (i) gravitons on this path, or (ii) gluons of $\mathbf{1} \backslash\left\{x_{r}\right\}$ and $\mathbf{2}$, through type-2 lines whose arrows point towards the direction of the root $x_{1}$.

\section{B Spinor helicity formalism and helpful identities}

We now review useful properties of spinor-helicity formalism, the Parke-Taylor formula and the Hodges formula. Helpful identities are displayed and proved in this section.

\section{B.1 Spinor helicity formalism}

In spinor-helicity formalism, momentum $k_{i}^{\mu}$ of an on-shell massless particle $i$ are mapped to two copies of two-component Weyl spinors $\lambda_{i}^{a} \widetilde{\lambda}_{i}^{\dot{a}}$. Polarizations for negative- and positivehelicity gluons are respectively expressed as

$$
\epsilon_{i}^{-}(\xi) \rightarrow \frac{\lambda_{i}^{a} \widetilde{\lambda}_{\xi}^{\dot{a}}}{[\xi, i]}, \quad \epsilon_{i}^{+}(\xi) \rightarrow \frac{\lambda_{\xi}^{a} \widetilde{\lambda}_{i}^{\dot{a}}}{\langle\xi, i\rangle},
$$


where a normalization factor $\sqrt{2}$ (see e.g. [43]) has been absorbed for convenience. In the above expression, $\xi^{\mu}$ is the reference momentum which reflects the choice of gauge and the spinor products are defined by

$$
\langle i, j\rangle \equiv \epsilon_{a b} \lambda_{i}^{a} \lambda_{j}^{b}, \quad[i, j] \equiv \epsilon_{\dot{a} \dot{b}} \tilde{\lambda}_{i}^{\dot{a}} \tilde{\lambda}_{j}^{\dot{b}},
$$

where $\epsilon_{a b}$ and $\epsilon_{\dot{a} \dot{b}}$ are totally antisymmetric tensors. With this expression, the Lorentz contraction of two vectors are given by:

$$
k_{a} \cdot k_{b}=\frac{1}{2}\langle a, b\rangle[b, a], \quad k_{a} \cdot \epsilon_{b}^{-}(q)=\frac{\langle a, b\rangle[q, a]}{[q, b]}, \quad k_{a} \cdot \epsilon_{b}^{+}(q)=\frac{[a, b]\langle q, a\rangle}{\langle q, b\rangle} .
$$

Helpful properties in spinor-helicity formalsim are displayed as follows.

- Antisymmety of the spinor products:

$$
\langle a, b\rangle=-\langle b, a\rangle, \quad[c, d]=-[d, c]
$$

When $a=b$ or $c=d$, we have $\langle a, a\rangle=0$ and $[c, c]=0$.

- Schouten identity:

$$
\langle a, b\rangle\langle c, d\rangle=\langle a, c\rangle\langle b, d\rangle+\langle b, c\rangle\langle d, a\rangle, \quad[a, b][c, d]=[a, c][b, d]+[b, c][d, a]
$$

- As a result of Schouten identity, we have the eikonal identity

$$
\sum_{i=j}^{k-1} \frac{\langle i, i+1\rangle}{\langle i, q\rangle\langle q, i+1\rangle}=\frac{\langle j, k\rangle}{\langle j, q\rangle\langle q, k\rangle} .
$$

- Momentum conservation for an $n$-point amplitude:

$$
\sum_{\substack{i \neq j, k \\ i=1}}^{n}[j, i]\langle i, k\rangle=0 .
$$

\section{B.2 Useful identities for Parke-Taylor factors}

As a consequence of the eikonal identity, Parke-Taylor factors satisfy the following property

$$
\begin{aligned}
\sum_{\boldsymbol{\sigma}} \frac{1}{(1, \ldots, j, \boldsymbol{\sigma} \in\{j+1, \ldots, r-1\} \amalg\{a\}, r)} & \equiv \sum_{\boldsymbol{\sigma}} \frac{1}{\left(1,\left.\{2, \ldots, r-1\} \amalg\{a\}\right|_{j \prec a}, r\right)} \\
& =\frac{1}{(1,2, \ldots, r)} \frac{\langle j, r\rangle}{\langle j, a\rangle\langle a, r\rangle},
\end{aligned}
$$

where $a$ is extracted out from the Parke-Taylor factors. Property (B.8) is further extended to the following more general property

$$
\begin{aligned}
\sum_{\boldsymbol{\sigma}} \frac{1}{\left(1, \ldots, j, \boldsymbol{\sigma} \in\{j+1, \ldots, r-1\} \amalg\left\{a_{1}, a_{2}, \ldots, a_{l}\right\}, r\right)} & \equiv \sum_{\boldsymbol{\sigma}} \frac{1}{\left(1,\left.\{2, \ldots, r-1\} \amalg\left\{a_{1}, a_{2}, \ldots, a_{l}\right\}\right|_{\left.j \prec a_{1}, r\right)}\right.} \\
& =\frac{1}{(1,2, \ldots, r)} \frac{\langle j, r\rangle}{\left\langle j, a_{1}\right\rangle\left\langle a_{1}, a_{2}\right\rangle \ldots\left\langle a_{l}, r\right\rangle} \\
& \equiv \frac{1}{(1,2, \ldots, r)} \frac{\langle j, r\rangle}{\left\langle j, a_{1}, \ldots, a_{l}, r\right\rangle},
\end{aligned}
$$

in which $\left\{a_{1}, \ldots, a_{l}\right\}$ are extracted out from the Parke-Taylor factors. Now we prove eq. (B.8) and eq. (B.9). 
Proof of eq. (B.8). The l.h.s. of eq. (B.8) can be rewritten as

$$
\begin{aligned}
\sum_{i=j}^{r-1} \frac{1}{(1, \ldots, i, a, i+1, \ldots, r)} & =\sum_{i=j}^{r-1} \frac{1}{(1, \ldots, i, i+1, \ldots, r)} \frac{\langle i, i+1\rangle}{\langle i, a\rangle\langle a, i+1\rangle} \\
& =\frac{1}{(1, \ldots, r)} \sum_{i=j}^{r-1} \frac{\langle i, i+1\rangle}{\langle i, a\rangle\langle a, i+1\rangle} \\
& =\frac{1}{(1, \ldots, r)} \frac{\langle j, r\rangle}{\langle j, a\rangle\langle a, r\rangle},
\end{aligned}
$$

where we have collected the coefficients for the common Parke-Taylor factor $1 /(1,2, \ldots, r)$ on the second line and applied the eikonal identity (B.6) on the third line. Thus eq. (B.8) has been proved.

Proof of eq. (B.9). When applying the identity (B.8) repeatedly, the l.h.s. of eq. (B.9) becomes

$$
\begin{aligned}
\sum_{\uplus} \frac{1}{(1, \ldots,}, j,\left.\left(\{j+1, \ldots, r-1\} \amalg\left\{a_{1}, a_{2}, \ldots, a_{l-1}\right\}\right) \amalg\left\{a_{l}\right\}\right|_{\left.a_{l-1} \prec a_{l}, r\right)} \\
\quad=\sum_{\varpi} \frac{\left\langle a_{l-1}, r\right\rangle}{\left(1, \ldots, j,\left(\{j+1, \ldots, r-1\} \amalg\left\{a_{1}, a_{2}, \ldots, a_{l-1}\right\}\right), r\right)} \frac{1}{\left\langle a_{l-1}, a_{l}\right\rangle\left\langle a_{l}, r\right\rangle} \\
\quad=\ldots \\
\quad=\sum_{\varpi} \frac{1}{(1,2, \ldots, r)}\left[\prod_{i=0}^{l-1} \frac{\left\langle a_{i}, r\right\rangle}{\left\langle a_{i}, a_{i+1}\right\rangle\left\langle a_{i+1}, r\right\rangle}\right]
\end{aligned}
$$

where $a_{0} \equiv j$. The product in the square brackets on the last line reads

$$
\frac{\langle j, r\rangle}{\left\langle j, a_{1}\right\rangle\left\langle a_{1}, r\right\rangle} \frac{\left\langle a_{1}, r\right\rangle}{\left\langle a_{1}, a_{2}\right\rangle\left\langle a_{2}, r\right\rangle} \cdots \frac{\left\langle a_{l-2}, r\right\rangle}{\left\langle a_{l-2}, a_{l-1}\right\rangle\left\langle a_{l-1, r\rangle}\right.} \frac{\left\langle a_{l-1, r\rangle}\right.}{\left\langle a_{l-1}, a_{l}\right\rangle\left\langle a_{l}, r\right\rangle}=\frac{\langle j, r\rangle}{\left\langle j, a_{1}, a_{2}, \ldots, a_{l}, r\right\rangle} .
$$

Hence the identity (B.9) has been proven.

When $j=1$, eq. (B.9) gives the KK relation for Parke-Taylor factors:

$$
\sum_{\boldsymbol{\sigma}} \frac{1}{(1,\{2, \ldots, r-1\} ш \boldsymbol{\beta}, r)}=(-1)^{|\boldsymbol{\beta}|} \frac{1}{\left(1,2, \ldots, r-1, r, \boldsymbol{\beta}^{T}\right)} .
$$

\section{B.3 Parke-Taylor formula and Hodges formula}

Color-ordered tree level YM amplitude $A^{(i, j)}(1,2, \ldots, n)$ with two negative-helicity gluons $i$ and $j$ satisfies the famous Parke-Taylor [31] formula

$$
A^{(i, j)}(1,2, \ldots, n)=\frac{\langle i, j\rangle^{4}}{(1,2, \ldots, n)} .
$$

Single-trace EYM amplitudes with two negative-helicity particles $i, j(i, j$ can be two gluons or one graviton plus one gluon) can be expressed by the following Hodges determinant form [25]:

$$
A^{(i, j)}(1,2, \ldots, r \| \mathrm{H}) \sim \frac{\langle i, j\rangle^{4}}{(1,2, \ldots, r)} \operatorname{det}\left[\phi_{\mathrm{H}^{+}}\right]
$$


where a normalization factor has been neglected, $1,2, \ldots, r$ are gluons in the trace $\mathbf{1}, \mathbf{H}$ denotes the graviton set, $\mathrm{H}^{+}$denotes the set of positive-helicity gravitons. In the above expression, the $\left|\mathrm{H}^{+}\right| \times\left|\mathrm{H}^{+}\right|$Hodges matrix $\phi_{\mathrm{H}^{+}}$is defined as

$$
\phi_{a b}=\frac{[a b]}{\langle a b\rangle} \quad(a \neq b), \quad \phi_{a a}=-\sum_{\substack{l \in 1 \cup \mathrm{H} \\ l \neq a}} \frac{[a l]\langle l \xi\rangle\langle l \eta\rangle}{\langle a l\rangle\langle a \xi\rangle\langle a \eta\rangle} \quad(a=b) .
$$

The spinors $\xi$ and $\eta$ represent a gauge freedom while $\phi_{a a}$ do not depend on it.

Open Access. This article is distributed under the terms of the Creative Commons Attribution License (CC-BY 4.0), which permits any use, distribution and reproduction in any medium, provided the original author(s) and source are credited.

\section{References}

[1] S. Stieberger and T.R. Taylor, New relations for Einstein-Yang-Mills amplitudes, Nucl. Phys. B 913 (2016) 151 [arXiv:1606.09616] [INSPIRE].

[2] D. Nandan, J. Plefka, O. Schlotterer and C. Wen, Einstein-Yang-Mills from pure Yang-Mills amplitudes, JHEP 10 (2016) 070 [arXiv:1607.05701] [INSPIRE].

[3] L. de la Cruz, A. Kniss and S. Weinzierl, Relations for Einstein-Yang-Mills amplitudes from the CHY representation, Phys. Lett. B 767 (2017) 86 [arXiv:1607.06036] [INSPIRE].

[4] O. Schlotterer, Amplitude relations in heterotic string theory and Einstein-Yang-Mills, JHEP 11 (2016) 074 [arXiv: 1608.00130] [INSPIRE].

[5] C.-H. Fu, Y.-J. Du, R. Huang and B. Feng, Expansion of Einstein-Yang-Mills amplitude, JHEP 09 (2017) 021 [arXiv:1702.08158] [INSPIRE].

[6] M. Chiodaroli, M. Günaydin, H. Johansson and R. Roiban, Explicit formulae for Yang-Mills-Einstein amplitudes from the double copy, JHEP 07 (2017) 002 [arXiv: 1703.00421] [INSPIRE].

[7] F. Teng and B. Feng, Expanding Einstein-Yang-Mills by Yang-Mills in CHY frame, JHEP 05 (2017) 075 [arXiv: 1703.01269] [INSPIRE].

[8] Y.-J. Du, B. Feng and F. Teng, Expansion of all multitrace tree level EYM amplitudes, JHEP 12 (2017) 038 [arXiv:1708.04514] [INSPIRE].

[9] L. Hou and Y.-J. Du, A graphic approach to gauge invariance induced identity, JHEP 05 (2019) 012 [arXiv: 1811.12653] [inSPIRE].

[10] Y.-J. Du and L. Hou, A graphic approach to identities induced from multi-trace Einstein-Yang-Mills amplitudes, JHEP 05 (2020) 008 [arXiv:1910.04014] [INSPIRE].

[11] Y.-J. Du and Y. Zhang, Gauge invariance induced relations and the equivalence between distinct approaches to NLSM amplitudes, JHEP 07 (2018) 177 [arXiv:1803.01701] [INSPIRE].

[12] Y.-J. Du and C.-H. Fu, Explicit BCJ numerators of nonlinear sigma model, JHEP 09 (2016) 174 [arXiv: 1606.05846] [inSPIRE].

[13] J.J.M. Carrasco, C.R. Mafra and O. Schlotterer, Abelian Z-theory: NLSM amplitudes and $\alpha^{\prime}$-corrections from the open string, JHEP 06 (2017) 093 [arXiv:1608.02569] [INSPIRE]. 
[14] Y.-J. Du and F. Teng, BCJ numerators from reduced Pfaffian, JHEP 04 (2017) 033 [arXiv: 1703.05717] [INSPIRE].

[15] Z. Bern, J.J.M. Carrasco and H. Johansson, New relations for gauge-theory amplitudes, Phys. Rev. D 78 (2008) 085011 [arXiv:0805.3993] [InSPIRE].

[16] B. Feng, X.-D. Li and R. Huang, Expansion of EYM amplitudes in gauge invariant vector space, Chin. Phys. C 44 (2020) 123104 [arXiv:2005.06287] [InSPIRE].

[17] B. Feng, X. Li and K. Zhou, Expansion of Einstein-Yang-Mills theory by differential operators, Phys. Rev. D 100 (2019) 125012 [arXiv:1904.05997] [INSPIRE].

[18] J. Plefka and W. Wormsbecher, New relations for graviton-matter amplitudes, Phys. Rev. D 98 (2018) 026011 [arXiv: 1804.09651] [INSPIRE].

[19] K. Zhou and G.-J. Zhou, Note on scalar-graviton and scalar-photon-graviton amplitudes, Eur. Phys. J. C 80 (2020) 930 [arXiv:2007.05910] [InSPIRE].

[20] F. Cachazo, S. He and E.Y. Yuan, Scattering equations and Kawai-Lewellen-Tye orthogonality, Phys. Rev. D 90 (2014) 065001 [arXiv:1306.6575] [InSPIRE].

[21] F. Cachazo, S. He and E.Y. Yuan, Scattering of massless particles in arbitrary dimensions, Phys. Rev. Lett. 113 (2014) 171601 [arXiv:1307.2199] [INSPIRE].

[22] F. Cachazo, S. He and E.Y. Yuan, Scattering of massless particles: scalars, gluons and gravitons, JHEP 07 (2014) 033 [arXiv:1309.0885] [INSPIRE].

[23] F. Cachazo, S. He and E.Y. Yuan, Einstein-Yang-Mills scattering amplitudes from scattering equations, JHEP 01 (2015) 121 [arXiv: 1409.8256] [INSPIRE].

[24] F. Cachazo, S. He and E.Y. Yuan, Scattering equations and matrices: from Einstein to Yang-Mills, DBI and NLSM, JHEP 07 (2015) 149 [arXiv:1412.3479] [INSPIRE].

[25] Y.-J. Du, F. Teng and Y.-S. Wu, Direct evaluation of n-point single-trace MHV amplitudes in 4d Einstein-Yang-Mills theory using the CHY formalism, JHEP 09 (2016) 171 [arXiv: 1608.00883] [INSPIRE].

[26] A. Hodges, A simple formula for gravitational MHV amplitudes, arXiv:1204.1930 [INSPIRE].

[27] B. Feng and S. He, Graphs, determinants and gravity amplitudes, JHEP 10 (2012) 121 [arXiv:1207.3220] [INSPIRE].

[28] K.G. Selivanov, Gravitationally dressed Parke-Taylor amplitudes, Mod. Phys. Lett. A 12 (1997) 3087 [hep-th/9711111] [INSPIRE].

[29] K.G. Selivanov, SD perturbiner in Yang-Mills + gravity, Phys. Lett. B 420 (1998) 274 [hep-th/9710197] [INSPIRE].

[30] Z. Bern, A. De Freitas and H.L. Wong, On the coupling of gravitons to matter, Phys. Rev. Lett. 84 (2000) 3531 [hep-th/9912033] [INSPIRE].

[31] S.J. Parke and T.R. Taylor, An amplitude for $n$ gluon scattering, Phys. Rev. Lett. 56 (1986) 2459 [INSPIRE].

[32] Z. Xu, D.-H. Zhang and L. Chang, Helicity amplitudes for multiple Bremsstrahlung in massless non-Abelian gauge theories, Nucl. Phys. B 291 (1987) 392 [INSPIRE].

[33] H. Kawai, D.C. Lewellen and S.-H. Henry Tye, A relation between tree amplitudes of closed and open strings, Nucl. Phys. B 269 (1986) 1 [INSPIRE]. 
[34] R. Britto, F. Cachazo and B. Feng, New recursion relations for tree amplitudes of gluons, Nucl. Phys. B 715 (2005) 499 [hep-th/0412308] [INSPIRE].

[35] R. Britto, F. Cachazo, B. Feng and E. Witten, Direct proof of tree-level recursion relation in Yang-Mills theory, Phys. Rev. Lett. 94 (2005) 181602 [hep-th/0501052] [InSPIRE].

[36] R. Kleiss and H. Kuijf, Multi-gluon cross-sections and five jet production at hadron colliders, Nucl. Phys. B 312 (1989) 616 [InSPIRE].

[37] Z. Bern, L.J. Dixon, M. Perelstein and J.S. Rozowsky, Multileg one loop gravity amplitudes from gauge theory, Nucl. Phys. B 546 (1999) 423 [hep-th/9811140] [INSPIRE].

[38] N. Arkani-Hamed, F. Cachazo, C. Cheung and J. Kaplan, A duality for the S matrix, JHEP 03 (2010) 020 [arXiv: 0907.5418] [INSPIRE].

[39] D. Nguyen, M. Spradlin, A. Volovich and C. Wen, The tree formula for MHV graviton amplitudes, JHEP 07 (2010) 045 [arXiv: 0907.2276] [INSPIRE].

[40] C. Boucher-Veronneau and A.J. Larkoski, Constructing amplitudes from their soft limits, JHEP 09 (2011) 130 [arXiv:1108.5385] [InSPIRE].

[41] D. Nandan and C. Wen, Generating all tree amplitudes in $N=4$ SYM by inverse soft limit, JHEP 08 (2012) 040 [arXiv: 1204.4841] [INSPIRE].

[42] L.J. Dixon, J.M. Henn, J. Plefka and T. Schuster, All tree-level amplitudes in massless QCD, JHEP 01 (2011) 035 [arXiv: 1010.3991] [INSPIRE].

[43] B. Feng and M. Luo, An introduction to on-shell recursion relations, Front. Phys. (Beijing) 7 (2012) 533 [arXiv:1111.5759] [INSPIRE]. 